\title{
Prävalenz und klinische Relevanz enoraler Mykosen und Dermatomykosen bei Bewohnern in pflegerischen Versorgungseinrichtungen
}

\author{
INAUGURAL-DISSERTATION \\ zur Erlangung des Doktorgrades
}

für Zahnheilkunde

der Medizinischen Fakultät der

Georg-August-Universität zu Göttingen

\author{
vorgelegt von \\ Klaus-Peter Wojak \\ aus \\ Detmold
}

Göttingen 2016 
Dekan:

1. Berichterstatter:

2. Berichterstatter:

3. Berichterstatter:
Prof. Dr. H. K. Kroemer

Prof. Dr. F. Nauck

Prof. Dr. U. Reichard

Prof. Dr. R. Mausberg

Tag der mündlichen Prüfung: 19.09.2016 


\section{Inhaltsverzeichnis}

1 Einleitung....................................................................................................................... 1

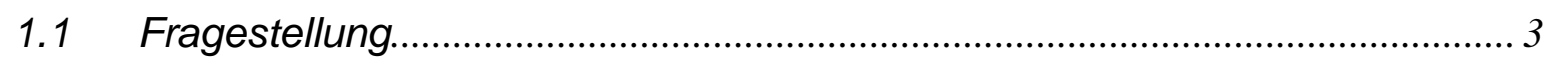

2 Material und Methoden ...................................................................................................... 5

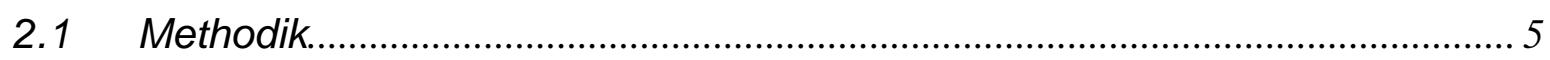

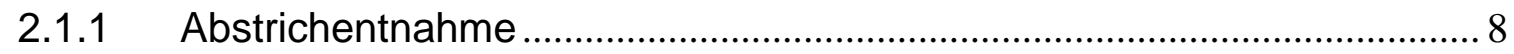

2.1.2 Herstellung von Agarböden ...................................................................... 8

2.1.3 Anlage von Kulturen .......................................................................

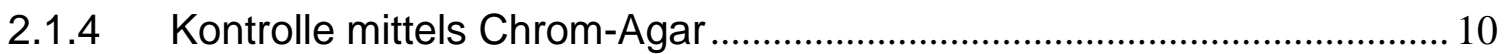

2.1.5 Differenzierung mittels MALDI-TOF .......................................................... 11

2.1.6 Suszeptibilitätsprüfung .............................................................................. 13

2.1.7 Statistische Methoden .......................................................................... 16

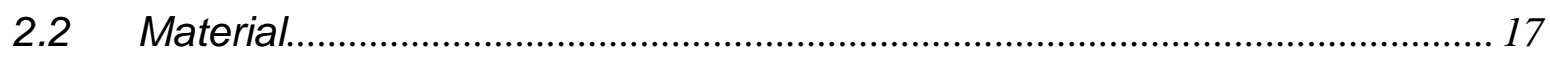

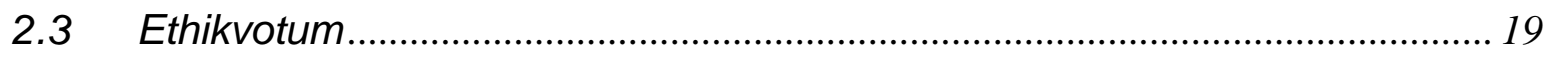

3 Ergebnisse ................................................................................................................... 20

3.1 Ergebnisse der Befunderhebungsbögen ....................................................... 20

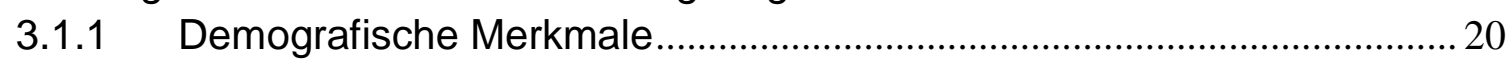



3.1.3 Klinische Symptome und Inspektion der Abstrichareale ......................... 28

3.2 Ergebnisse mikrobiologischer Untersuchungen ..............................................33

3.2.1 Häufigkeit der mykologischen Befunde ........................................................ 33

3.2.2 Vergleich Untersuchungs- und Kontrollgruppe ............................................. 35

3.2.3 Mykologische Auswertung ......................................................................... 39

3.2.4 Daten der Suszeptibilitätsprüfung ............................................................ 45

3.3 Korrelation von mykologischen Befunden und klinischen Symptomen ........ 48

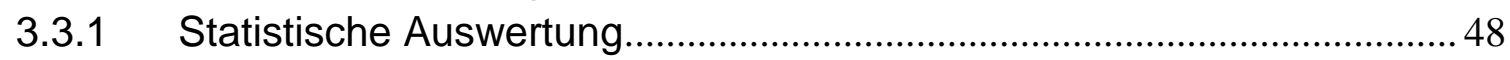

3.3.2 Logistische Regression ......................................................................... 57

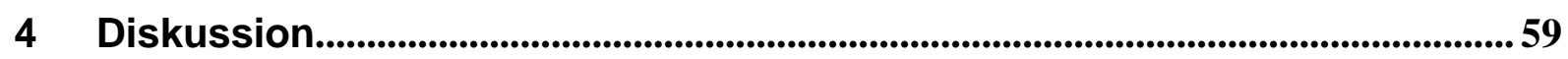

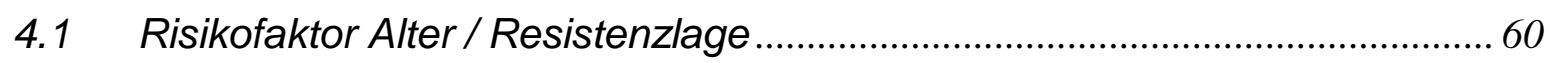

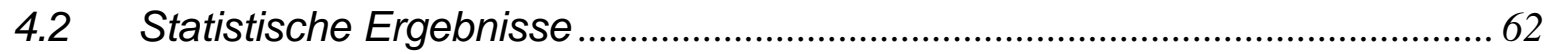

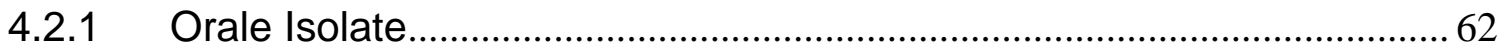

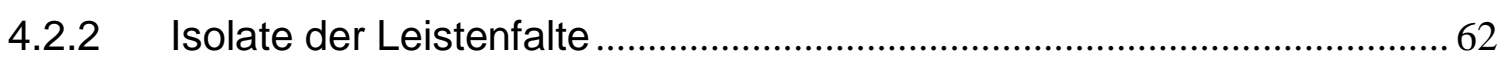

4.2.3 Isolate der Zehenzwischenräume ................................................................ 63

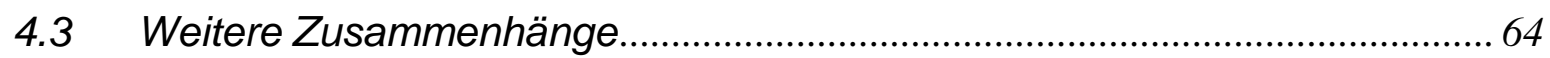

4.3.1 Hygienemaßnahmen/ Zahnprothesen als Risikofaktoren ........................6 65

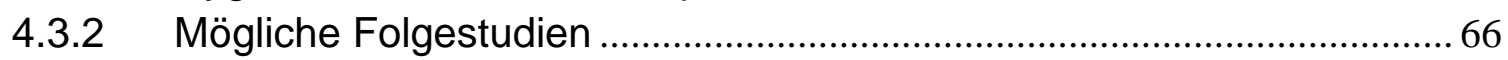

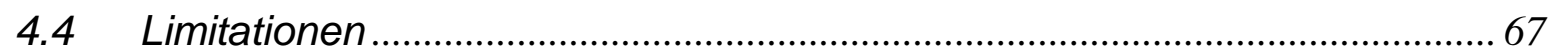




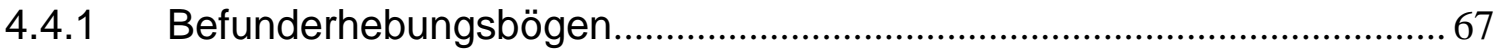

4.4.2 Rekrutierung der Probanden/ Compliance .............................................67

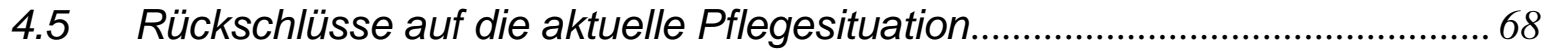

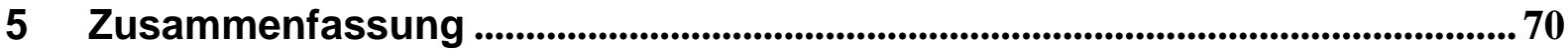

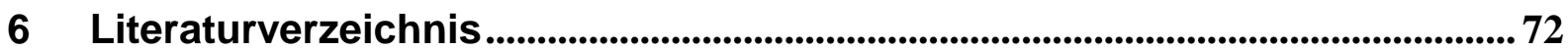

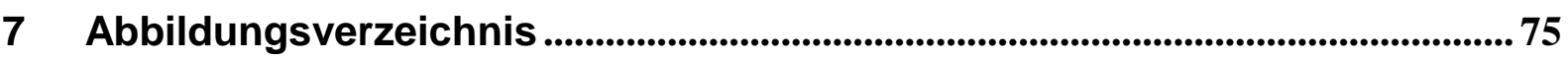

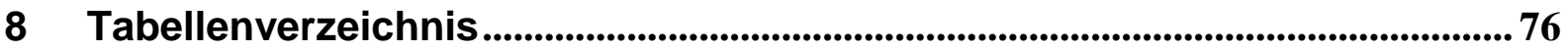

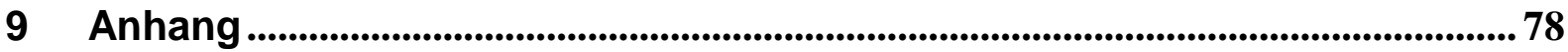

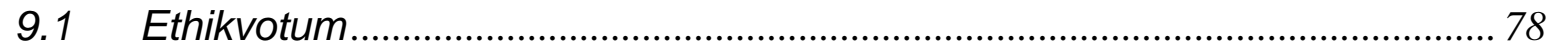

9.2 Befunderhebungsbogen der Bewohner/-innen der Pflegeeinrichtungen...... 79

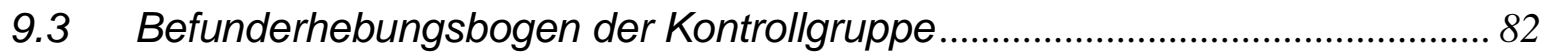

9.4 Patienteninformationen (Probanden, Betreuer und Kontrollgruppe).............. 83

9.5 Einverständniserklärungen (Probanden, Betreuer und Kontrollgruppe)....... 89

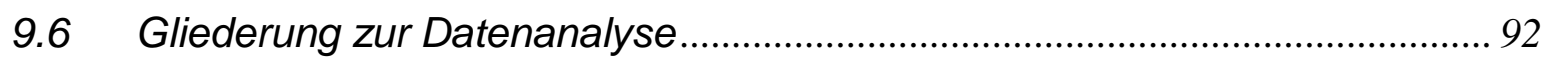




\section{$1 \quad$ Einleitung}

Enorale Symptome und Befunde gehören zu den vernachlässigten Problemen in der Medizin. Eine vorangegangene Pilotstudie der Klinik für Palliativmedizin der Universitätsmedizin Göttingen zeigte, dass bei Patienten mit weit fortgeschrittenen Tumorerkrankungen häufig eine enorale Beschwerdesymptomatik besteht und deutlich häufiger als in vorangegangenen epidemiologischen Studien, in der die Symptome mittels Fremdeinschätzung erfasst wurden, gefunden wurde. Mundtrockenheit und Geschmacksstörungen standen hierbei im Vordergrund. Des Weiteren wurde festgestellt, dass orale Beschwerden mit einer hohen Prävalenz oraler Mykosen signifikant in Verbindung gebracht werden konnten (Kordestani 2009; Alt-Epping et al. 2012). Weiterführende Untersuchungen zu oralen Komplikationen bei Palliativpatienten mit fortgeschrittenen Tumorerkrankungen sind jedoch selten (Sweeney und Bagg 2000).

Dass nicht nur bei Palliativpatienten, sondern bei älteren Personen überhaupt die Prävalenz einer oralen Mykose zunimmt, konnten Laurent et al. 2011 zeigen. Hof (2010), Pfaller und Diekema (2007) konnten ebenfalls feststellen, dass mit dem Alter auch die mykologischen Befunde zu variieren scheinen und Non-albicans-Spezies eine zunehmend bedeutende Rolle einnehmen.

Weitere Einblicke in die Literatur zeigen, dass eine große Anzahl der Studien auf dem Hintergrund einer veränderten Immunabwehr wie bei HIV-Patienten und/oder aufgrund von invasiven Mykosen angelegt wurden (Peman und Salavert 2012; Albougy und Naidoo 2002; Cassone und Cauda 2012), da besonders bei HIV-/AIDSerkrankten Patienten ein signifikanter Zusammenhang zu einer Pilzbesiedlung aufgezeigt werden konnte (Martinez Machin et al. 1997; Cassone und Cauda 2012).

Ebenso konnte ein häufiger Pilzbefall bei Patienten aus stationären Versorgungseinrichtungen, welche durch die Schwere oder Inkurabilität einer vorausgehenden Erkrankung gekennzeichnet sind, beobachtet werden (Bagg et al. 2003).

Zu der oralen Symptomlast sowie dem mikrobiologischen Milieu gesunder älterer Probanden fehlen Untersuchungen, da dieses Probandenkollektiv meist im klinischen 
Alltag nicht angetroffen wird, wodurch sich Untersuchungen an stationären Patienten zwangsläufig auf ein medizinisch und pflegerisch vorbelastetes Kollektiv stützen.

Untersuchungen zur Situation der Mundhygiene und Symptomlast institutionalisierter älterer Probanden in stationären Pflegeeinrichtungen ergaben eine Häufung des subjektiven Gefühls eines trockenen Mundes, Geschmacksstörungen sowie einer verminderten Speichelflussrate (Glazar et al. 2010), jedoch ohne Aussagen zum mikrobiologischen Milieu bei den betroffenen Probanden.

Der Vergleich der Symptomlast von Probanden mit einer nachgewiesenen Candidabesiedlung zeigt jedoch eine deutliche Überschneidung der in Studien gefundenen oralen Symptome (Yamamoto 2010).

Dabei spielen sowohl allgemeine prädisponierende Faktoren für eine orale Candidiabesiedlung wie das Tragen einer Prothese (Dorko et al. 2001), verminderter Speichelfluss, schlechte Mundhygiene, aber auch weitere Faktoren wie die Einnahme von Antibiotika, ein Diabetes mellitus, eine Immunsuppression oder maligne Tumoren eine Rolle (Krishnan 2012).

Aufgrund der bisher unzureichenden Literatur und des Fehlens empirischer Daten zu dem Nachweis mykologischer Befunde gesunder älterer Bevölkerungsgruppen ist das Ziel dieser klinischen Studie die Untersuchung der Prävalenz und klinischen Relevanz enoraler Mykosen und Dermatomykosen bei Bewohnern/-innen in stationären Pflegeeinrichtungen. Dabei handelt es sich um ein wahrscheinlich unterschätztes Problem. 


\subsection{Fragestellung}

Es soll im Folgenden bei Menschen, die in stationären Pflegeeinrichtungen betreut werden (Altenpflege- und Demenzpflegeeinrichtung) untersucht werden, welche sowohl subjektiven Symptome als auch klinischen und mikrobiologischen Befunde des Mundraumes bei älteren, teils medizinisch erkrankten, teils gesunden Pflegeheimbewohnern/-innen vorliegen und ob und inwieweit diese miteinander oder mit weiteren Faktoren wie Alter oder etwaigen Grunderkrankungen sowie eingenommenen Medikamenten korrelieren.

Infolge der hohen Inzidenz von mykologischen Befunden im Alter, wie sie bereits in der Literatur beschrieben wurden (Hof 2010), wird den mikrobiologischen Pilznachweisen in dieser Studie eine besondere Aufmerksamkeit gewidmet. Hierzu werden Bewohner/-innen aus zwei Göttinger Pflegeheimen (Pflegeheim 1 und Pflegeheim 2) sowie Bewohner/-innen eines Göttinger Demenzpflegeheims (Pflegeheim 3) als Probanden in die Studie aufgenommen. Als Kontrollgruppe wurden junge Medizinstudentinnen und -studenten der Universitätsmedizin Göttingen hinzugezogen.

Im Einzelnen sollten folgende Fragen beantwortet werden:

- Welche enoralen (und dermatologischen) Symptome werden in unterschiedlichen stationären Pflegeeinrichtungen, die betagte Menschen betreuen, durch die Probanden angegeben?

- Welche anamnestischen Co-Faktoren (Nahrung, Medikamente, Hygiene) lassen sich eruieren?

- Wie unterscheiden sich die Patienten und Bewohner/-innen der verschiedenen Versorgungseinrichtungen bezüglich ihrer Symptomverteilung voneinander?

- Mit welcher Häufigkeit wird in den o. g. stationären Pflegeeinrichtungen eine enorale Candidose und/oder Dermatomykose gefunden?

- Wie unterscheiden sich die einzelnen pflegerischen Versorgungseinrichtungen (stationäre Pflege, stationäre Demenzpflege) voneinander?

- Welche Spezies lassen sich bei Probandengruppe und Kontrollgruppe finden und wie ist deren Resistenzlage? 
- In welchem Ausmaß lässt sich das Auftreten enoraler oder dermatologischer Symptome mit dem mikrobiologischen Nachweis einer Mykose korrelieren?

- Wie unterscheiden sich die Probandengruppen von einem Vergleichskollektiv mit gesunden, nicht altersgematchten Probanden?

- Gibt es Korrelationen zwischen dem Alter, Komorbiditäten, der Pflegebedürftigkeit (ADL) der Patientengruppen etc. und der Häufigkeit mykologischer Befunde und/oder der Lokalisation der mikrobiologischen Pilznachweise? 


\section{Material und Methoden}

\section{$2.1 \quad$ Methodik}

Angelegt als multizentrische Studie der Klinik für Palliativmedizin (Universitätsmedizin Göttingen, Direktor Prof. Dr. F. Nauck), in Zusammenarbeit mit der Abteilung Medizinische Mikrobiologie (Direktor Prof. Dr. U. Groß) und drei Göttinger stationären Pflegeeinrichtungen (Seniorenpflegezentrum „Haus Drei Linden“ in Bovenden (Pflegeheim 1), Seniorenzentrum Göttingen (Pflegeheim 2) und Luisenhof „Zentrum für Pflege und Betreuung“ in Göttingen (Pflegeheim 3) unter der Förderung des Nationalen Referenzzentrums für Systemische Mykosen (NRZSM) mit Sitz im Institut für Klinische Mikrobiologie in Göttingen wurden im Rahmen der vorliegenden klinischen Studie durch folgende Maßnahmen Informationen aufgenommen, gesammelt und analysiert:

Ab dem 31.03.2009 wurden Bewohner/-innen der beiden Pflegeeinrichtungen (Seniorenpflegezentrum Bovenden und Seniorenzentrum Göttingen) gebeten, an der Studie teilzunehmen. Die Probanden wurden mündlich sowie schriftlich über das Vorgehen aufgeklärt. Erst nach schriftlicher Einverständniserklärung der Bewohner/innen bzw. nach vorheriger telefonischer sowie schriftlicher Aufklärung der zuständigen betreuenden Personen wie auch deren schriftlichen Einverständniserklärung wurde die Person die sich bereiterklärt hatten an der Studie teilzunehmen, befragt.

Zur Strukturierung der Befragung war durch den Untersucher in Zusammenarbeit mit G. Ungermann ein Befunderhebungsbogen konzipiert worden (siehe Anhang), in dem die Angaben der Studienteilnehmer systematisch dokumentiert wurden.

Hierbei handelte es sich $u$. a. um Fragen zur oralen Beschwerdesymptomatik, Vorbehandlungen mit Auswirkungen auf den derzeitigen Gesundheitszustand, der Mundhygiene sowie der Digestion und Körperpflege.

Alle potentiellen Studienteilnehmer wurden vor der Untersuchung über den Ablauf und die Dauer der Untersuchung informiert und aufgeklärt.

Die Auswahl der Teilnehmer wurde zuvor von der Pflegeheimleitung nach einem Zufallsprinzip durchgeführt, bewusst ohne weitere Auswahlkriterien wie Mobilität oder Alter, um einen Überblick über die Situation und die betreuten Personen in der 
ganzen Pflegeeinrichtung zu ermöglichen. Einzige Voraussetzung war jedoch eine bisherige Aufenthaltsdauer in der betreffenden Pflegeeinrichtung von mehr als 72 Stunden.

Zusätzliche anamnestische Informationen wie die aktuelle Medikation, weitere Erkrankungen sowie die Pflegestufe (orig. ADL-scale: acitivties of daily life bzw. ATL: Aktivitäten des täglichen Lebens) wurden den Akten der Pflegeeinrichtung entnommen.

War es nicht möglich den Patienten zu befragen, z.B. aufgrund von Grunderkrankungen wie u.a. stark fortgeschrittener Demenz, so wurde zur Beantwortung der Fragen, so weit möglich, das zuständige Pflegepersonal hinzugezogen.

Nach dem Gespräch mit den Probanden wurden zur mikrobiologischen Diagnostik Abstriche mit einem Watteträger von der Mundschleimhaut, aus der Leistenfalte wie auch aus dem Zehenzwischenraum entnommen.

Diese Abstriche wurden von dem Doktoranden in der Abteilung Medizinische Mikrobiologie der Universitätsmedizin Göttingen gesammelt und unter Einschluss der unten im Detail aufgeführten speziellen mikrobiologischen Verfahren untersucht.

Als Kontrollgruppe wurden 47 Medizinstudierende hinzugezogen, die sich im Rahmen des mikrobiologischen Praktikums bzw. nach dem Kurs bereit erklärt haben, Abstriche an den vorgegebenen Körperregionen abnehmen zu lassen. Die Proben der Kontrollgruppe wurden ebenso wie die der Untersuchungsgruppe mit denselben mikrobiologischen Untersuchungsmethoden analysiert.

Parallel zur vorliegenden Untersuchung an ambulant pflegerisch betreuten Personen wurden im Rahmen einer weiteren Promotionsarbeit mit derselben Methodik Patienten aus klinischen Versorgungseinrichtungen untersucht.

Hierzu wurden Patienten der Klinik für Palliativmedizin sowie der Intensivstation der Universitätsmedizin Göttingen und Patienten des Geriatrischen Zentrums des Evangelischen Krankenhauses in Göttingen- Weende untersucht. Die aus diesen Untersuchungen resultierenden Ergebnisse werden in der Dissertationsarbeit von Gertrud Ungermann dargestellt.

Aufgrund der ähnlichen Konzeption dieser beiden Studien in Bezug auf die (ambulante versus stationäre) Auswahl besonders vulnerabler Patientengruppen 
sowie in Bezug auf die Erfassungsmethodik und die mikrobiologische Auswertung wurden Teile der wissenschaftlichen Arbeit in einer gemeinsamen Arbeitsgruppe erarbeitet. Die Proben und anamnestischen Daten wurden getrennt voneinander gesammelt. Die mikrobiologischen Untersuchungen und Datenerhebungen sowie eine Einleitung in die statistische Auswertung wurden größtenteils gemeinsam durchgeführt. Die Auswertung der Daten wiederum wurde getrennt vorgenommen.

Wie die Bewohner/-innen der Pflegeeinrichtungen wurden nur Probanden der Kontrollgruppe in die Studie aufgenommen, die zuvor mündlich und schriftlich über den Ablauf und das Vorgehen aufgeklärt wurden und schriftlich ihre Einverständniserklärung gegeben haben. Ein eigens für die Kontrollgruppe angefertigter Befunderhebungsbogen (siehe Anhang) diente zur Datenerhebung. Im anschließenden Verlauf glich die Probenentnahme derjenigen bei den Bewohnern/innen in den Pflegeeinrichtungen.

Probandenbezogene Daten wurden mithilfe eines Zahlencodes pseudonymisiert.

Die Befragungen in den Pflegeeinrichtungen wie auch die Entnahme der Proben fanden meistens vormittags während der Woche statt, so dass das Pflegepersonal in der Ausübung der täglichen Arbeit möglichst nicht gestört wurde.

Zudem zeigte sich, dass sich dieser Zeitraum gut für die Probenentnahme, Befragung der Probanden sowie die Mitarbeit des Studienteilnehmers eignete. Die Besuchszeiten wurden stets gemieden.

$\mathrm{Zu}$ jedem Zeitpunkt war es möglich die Befragung wie auch die Probenentnahme abgebrochen werden.

Waren die Probanden oder deren Betreuer mit der Teilnahme an der Studie nicht einverstanden, wurde dies zu Kenntnis genommen und respektiert.

Zusätzlich zu der oralen Abstrichentnahme wurde vom Doktoranden die Mundhöhle (Schleimhaut, Zunge, Gaumen, Dentition) bestmöglich inspiziert und der Befund dokumentiert.

Ebenso wurde bei der Abstrichentnahme aus Leistenfalte- und Fußregion verfahren und Veränderungen der Abstrichregion dokumentiert. 


\subsubsection{Abstrichentnahme}

Die Abstrichentnahme mit Universal-Kunststoffwatteträgern wurde mit dem Abstrichsystem der Firma COPAN durch den Doktoranden oder selten mit Hilfe des Pflegepersonals durchgeführt.

Vor der Probenentnahme wurden alle Teilnehmer nochmals über das Vorgehen informiert und nur bei ausdrücklichem Einverständnis des Teilnehmers wurde mit der Untersuchung begonnen bzw. fortgefahren.

Im Bereich der Mundhöhle wurden Abstriche mit dem sterilen Watteträger von Bereichen der Wangenschleimhaut, dem Gaumen und der Zunge genommen.

Des Weiteren wurden eine Probe von der Leistenfalte wie auch eine Probe von verschiedenen Zehenzwischenräumen genommen. Jede Probe wurde direkt nach der Abstrichentnahme beschriftet und durch einen Zahlencode pseudonymisiert.

Um eine mögliche spätere Wiederverwendung und damit eine lange Aufbewahrungszeit zu ermöglichen, wurden alle Abstriche bei ca. $4{ }^{\circ} \mathrm{C}$ kühl gelagert. Während der Abstrichentnahme wurden die Abstrichareale sowie angrenzende Regionen bestmöglich inspiziert.

\subsubsection{Herstellung von Agarböden}

Die Nährböden wurden (aus Kostengründen) vom Doktoranden selber speziell für die mykologische Untersuchungen hergestellt. Als Suspensionslösung wurde VEWasser verwendet, das von Mitarbeitern der Abteilung Medizinische Mikrobiologie bereitgestellt wurde.

Der Sabouraud-Agar (Merck, Darmstadt, Deutschland) wurde nach den vom Hersteller angegebenen Dosierungen hergestellt und nach dem Autoklavieren für 20 Minuten bei $121^{\circ} \mathrm{C}$ mit Antibiotika (Gentamycin 0,04 g/l, Chloramphenicol 0,4 g/l) versetzt, um eine Bakterienvermehrung zu unterdrücken.

Gleichermaßen wurde in der Herstellung mit dem Malz-Agar (DIFCO, BD, New Jersey, USA) verfahren.

Gelagert wurden alle mit Agar befüllten Petrischalen in keimarmen Kühlschränken bei ca. $4^{\circ} \mathrm{C}$. 


\subsubsection{Anlage von Kulturen}

Nach der Abstrichentnahme wurden die Proben auf festen Nährböden ausgestrichen. Als Nährböden wurden Sabouraud-Agar (10 g/l mykologische Peptone, $40 \mathrm{~g} / \mathrm{l}$ Dextrose, $15 \mathrm{~g} / \mathrm{l}$ Agar; $65 \mathrm{~g}$ gelöst in $1 \mathrm{l}$ destilliertem Wasser, autoklaviert bei $121^{\circ} \mathrm{C}$ für 15 Minuten) und Malz-Agar (30 g/l Malzextrakt, $5 \mathrm{~g} / \mathrm{l}$ mykologische Peptone, $15 \mathrm{~g} / \mathrm{l}$ Agar; 50 g gelöst in 1 l destilliertem Wasser, autoklaviert bei $115^{\circ} \mathrm{C}$ für 10 Minuten) verwendet.

Auf jeweils einer Agarplatte wurde ein Abstrich der zu untersuchenden Gruppen ausgestrichen und in Brutschränken bei konstanter Temperatur inkubiert.

Die Proben der Mundhöhle, Leistenfalte und der Zehenzwischenräume wurden bei $37^{\circ} \mathrm{C}$ inkubiert und über einen Zeitraum von drei bis sieben Tagen beobachtet sowie das Koloniewachstum dokumentiert.

Konnte ein Wachstum in Form von Kolonien in Reinkultur beobachtet werden, wurden Zellen dieser Kolonie mit Hilfe des Stammerhaltungssystems Cryobank (MAST, Diagnostica, Reinfeld, Deutschland) aufbewahrt.

Hierzu wurden Zellen von mehreren Kolonien mit einer Einwegimpföse abgenommen, in die Kunststoffröhrchen eingebracht und pseudonymisiert bzw. zur weiteren Verwendung mit einem Zahlencode versehen. Um eine maximale Adhäsion der Zellen an den auf der Oberfläche chemisch behandelten Glaskügelchen zu ermöglichen, wurden die Kunststoffröhrchen geschwenkt. Anschließend wurde der Überstand der Konservierungslösung möglichst vollständig abpipettiert.

Alle Röhrchen des Stammerhaltungssystems wurden bei $-70^{\circ} \mathrm{C}$ eingefroren und somit für eine spätere Verwendung aufbewahrt.

Bei der makroskopischen Betrachtung der gewachsenen Kolonien zeigte sich eine typische Koloniemorphologie (weißliche, konvexe, nicht pigmentierte Kolonien).

Konnte kein Wachstum innerhalb des o.g. Zeitraumes beobachtet werden, wurde dies dokumentiert und die Nährbodenplatte entsorgt.

Die Proben der Leistenfalte und des Zehenzwischenraumes wurden zusätzlich nochmals auf Malz- und Sabouraud-Agar ausgestrichen und bei $26^{\circ} \mathrm{C}$ über 6 bis 8 Wochen inkubiert, um einen möglichen Befall von Dermatophyten nachweisen zu können. 
Ebenso wie die bei $37^{\circ} \mathrm{C}$ bebrüteten Proben wurden die Abstriche die bei $26^{\circ} \mathrm{C}$ angelegt wurden, im Wachstum beobachtet und die Anzahl der Kolonien dokumentiert. Es wurde in gleicher Weise das Koloniewachstum kategorisiert.

Kulturen, die auch nach 8 Wochen kein Wachstum zeigten, wurden dokumentiert und ebenfalls entsorgt.

Konnte ein Koloniewachstum verzeichnet werden, wurden Zellen dieser Kolonien ebenso mit einem fortlaufenden Zahlencode versehen und für eine spätere Verwendung eingefroren.

\subsubsection{Kontrolle mittels Chrom-Agar}

Um ausschließen zu können, dass keine Kolonien andere verdrängten und sie in ihrem Wachstum behinderten, wurden die Proben, bei denen ein Wachstum verzeichnet werden konnte, erneut auf Oxoid-Chrom-Agar Nährböden (Hampshire, England) ausgestrichen. Da sich Hefen unterschiedlicher Spezies auf diesem Nährboden farblich voneinander abgrenzen, kann somit zwischen verschieden Spezies innerhalb eines Abstrichmaterials differenziert werden. Diese Methode eignet sich für Mischkulturen sowie besonders zur Differenzierung von Candida albicans- und non-albicans-Spezies.

Unter anderem unterscheidet sich der Chrom-Agar von den anderen verwendeten Nährböden dadurch, dass das Antibiotikum bereits in den Materialien integriert ist und dass der Agar -im Gegensatz zu den o.g. Nährböden- durch kurzzeitige Erwärmung sterilisiert wird.

Bis zu dem Zeitpunkt der Lagerung der Kolonien im Stammerhaltungssystem wurden alle Nährböden mit o. g. Antibiotika versetzt. Da sich nach dem Auftauen der Kulturen und dem überimpfen der Nährböden keine weiteren Bakterien in den Proben befanden, konnte auf einen erneuten Einsatz von Antibiotika verzichtet werden. 


\subsubsection{Differenzierung mittels MALDI-TOF}

Matrix-unterstützte Laser-Desorption/ Ionisation (MALDI) mit Flugzeitanalysator (TOF; engl.: Time of Flight) ist ein Verfahren, das zur Massenspektrometrie entwickelt wurde. Für die Untersuchung der Proben wurde das Gerät Autoflex III (Bruker Daltonics, Billerica, USA) verwendet.

Der große Vorteil dieser relativ neuen Untersuchungsmethode ist die vergleichsweise schnelle, verlässliche und weniger kostenintensive Analyse im Gegensatz zu herkömmlichen Analyseverfahren.

Der Mechanismus beruht darauf, dass ein Analyt durch mehrere Arbeitsschritte in eine Matrix auf einem Objektträger eingebettet wird und anschließend mittels kurzer, energiereicher Laserimpulsen angeregt wird, in die Gasphase überzugehen und ionisiert zu werden.

Die abgelösten Teilchen von Matrix und Analyt werden im Vakuum des Massenspektrometers der Messung zugänglich. Die Partikel werden anschließend entsprechend ihrer Größe und Ladung in einem elektrischen Feld beschleunigt und in ein elektrisches Signal umgewandelt. Dieses durch die elektrischen Signale hervorgerufene Spektrum lässt Rückschlüsse auf die zu untersuchende Spezies zu. Die Messergebnisse des zu untersuchenden biologischen Materials werden anschließend durch die Flexcontrol-Software (Bruker) mit einer Datenbank abgeglichen, wodurch die Gattung (bzw. Genus) und Spezies (bzw. Art) ermittelt werden (Rodriguez-Tudela 2008).

Für jedes Feld eines Trägers werden zwei Messergebnisse ermittelt. Die Messwerte geben Aufschluss über die identifizierte Spezies (bzw. den Grad der Übereinstimmung der ermittelten Messwerte mit den Werten der Datenbank). Die Ergebnisse werden eingeteilt in „hohe Wahrscheinlichkeit der Speziesidentifikation“, „Sichere Genus-, wahrscheinliche Speziesidentifikation“, „wahrscheinliche Genusidentifikation“, „keine verlässliche Übereinstimmung“ (orig.: highly probable species identification, secure genus identification, probable species identification, probable genus identification, not reliable identification).

Ausschließlich Werte, die in den Bereich „hohe Wahrscheinlichkeit der Speziesidentifikation“ fielen und reproduzierbar sichere Angaben zur ermittelten Spezies lieferten, wurden für die weitere Datenanalyse verwendet. Ergebnisse, welche dieses Kriterium nicht erfüllten, wurden erneut untersucht und anschließend getestet. 
War das Ergebnis der Massenspektrometrie nicht eindeutig bzw. wurden zwei oder mehr Spezies in einer Probe gefunden, wurde die Probe aus dem dazugehörigen Abstrich überimpft und auf verschiedene Spezies untersucht. Die isolierten Spezies wurden erneut mittels des Massenspektrometers untersucht und bei eindeutigem Ergebnis als neue Proben dem Stammerhaltungssystem Cryobank (unter einer neuen Identifikationsnummer) beigefügt.

Die wenigen Spezies, die nicht von dem MALDI-TOF MS identifiziert werden konnten, wurden auf herkömmliche Weise ohne Massenspektrometrie identifiziert (Chen 2000).

Ursächlich für das Nichterkennen des Massenspektrometers waren Mischkulturen, die nicht getrennt werden konnten oder fehlende spezifische Informationen in der Datenbank zu selten beobachteten Isolaten.

Bei der Vorbereitung der „Targets“ für die Messung mittels des Massenspektrometers wurde genau nach den Herstellerangaben nach der „Ethanol/ Ameisensäure-Extraktion zur Mikroorganismen-Charakterisierung mit dem MALDI Biotyper" verfahren.

Hierzu wurden die zu untersuchenden Organismen über Nacht kultiviert. Als Matrix wurden anfangs Lösungen hergestellt, die nach den Vorgaben des Herstellers angefertigt wurden. Hierzu wurde die empfohlene Bruker-proportionierte Matrix verwendet, welche mit einem Basislösungsmittel nach sorgfältigem Mischen als Matrix genutzt werden konnte. Nach dem Pipettierschema wurde $1 \mathrm{ml}$ Basislösungsmittel des o. g. Protokolls, bestehend aus $500 \mu \mathrm{l} 50 \%$ Acetonitril, $25 \mu \mathrm{l}$ 2.5\% Tri-Fluor-Essigsäure und $457 \mu \mathrm{l}$ Aqua dest. hergestellt. Zu einem späteren Zeitpunkt wurden vorgefertigte Lösungen (gesättigte Lösungen von HCCA in BasisLösungsmittel) verwendet, da diese genauere Ergebnisse bezüglich der sicheren Speziesidentifikation bzw. des Identifikationsscores zeigten.

Die Matrix wurde bis zur Verwendung kühl und im Dunkeln gelagert. Vor der Verwendung wurde sie nach der Herstellerempfehlung einige Minuten sorgfältig vermischt (Vortex).

Bei der Probenvorbereitung wurde ebenfalls nach den Anwendungsempfehlungen vorgegangen, indem etwas biologisches Material der zu untersuchenden Kultur in $300 \mu \mathrm{l}$ Aqua dest. suspendiert wurde. Diese Probe wurde mit $900 \mu \mathrm{l}$ Ethanol (abs.) aufgefüllt, sorgfältig gemischt und bei maximaler Drehzahl für 2 Minuten zentrifugiert. 


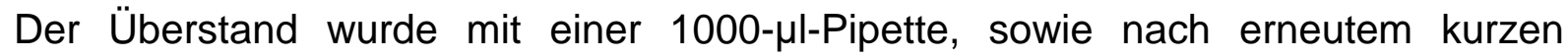
Zentrifugieren mit einer $20-\mu \mathrm{l}$-Pipette abpipettiert und verworfen.

Anschließend wurden die Pellets des biologischen Materials für mehrere Minuten bei Raumtemperatur getrocknet.

Im nächsten Arbeitsschritt wurden $50 \mu \mathrm{l}$ Ameisensäure hinzugegeben und sorgfältig gemischt. Die gleiche Menge Acetonitril wurde hinzugefügt und sorgfältig gemischt (Vortex). Nach erneutem Zentrifugieren für 2 Minuten bei maximaler Drehzahl wurde

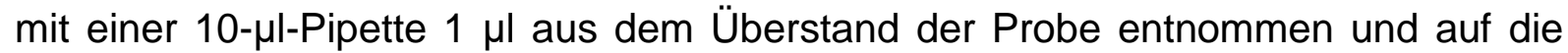
zuvor festgelegte Position des Mess-Targets pipettiert. Nach dem Trocknen der Probe wurde diese mit $1 \mu$ der Matrix-Lösung überdeckt. Nach dem Trocknen der hinzugefügten Matrix war die Probe der Messung mit dem MALDI-TOFMassenspektrometer zugänglich.

Um eine bestmögliche Qualität der wieder verwendbaren Targets für weitere Messungen zu ermöglichen, wurden diese, nachdem alle Positionen zur Messung mittels des Massenspektrometers belegt waren und die Messungen durchgeführt worden waren, nach dem „MALDI Biotyper target cleaning procedure“ gereinigt. Hierzu wurden die Stahlträger für 5 Minuten bei Zimmertemperatur mit Ethanol (70\%) bedeckt. Anschließend wurden die Platten unter fließendem Wasser abgespült und mit Ethanol (70\%) und Kimwipe-Tüchern abgewischt, bevor sie erneut unter fließendem Wasser mit Kimwipe-Tüchern gesäubert wurden. Im nächsten Schritt wurden die Platten mit jeweils $100 \mu$ l Tri-Fluor-Essigsäure (80\%) beschichtet und erneut mit Kimwipe-Tüchern gesäubert. Dieser Vorgang wurde unter einer Dunstabzugshaube durchgeführt. Im letzten Schritt wurden die Platten unter VEWasser abgespült und ebenfalls mit Kimwipe-Tüchern abgewischt. Wenige Minuten nach dem Trocknen der Platte bei Raumtemperatur konnten sie wieder für weitere Messzwecke verwendet werden.

\subsubsection{Suszeptibilitätsprüfung}

Die quantitative Resistenzbestimmung dient dazu, Angaben zu den Hemmkonzentrationen eines Antimykotikums, welches zur Therapie eines bestimmten Candidastammes verwendet werden soll, machen zu können.

Des Weiteren ermöglicht diese Methode eine Aussage über die Einstufung der Kultur -bezogen auf das Antimykotikum- als sensibel, intermediär oder resistent. 
Somit ist die Resistenztestung hilfreich für die Entscheidung bezüglich der korrekten Therapie und damit verbundenen Medikation.

Die Resistenztestung wurde nach dem Protokoll EDef 7.1 zur Resistenztestung von Hefepilzen des EUCAST (European Committee for Antimicrobial Susceptibility Testing) durchgeführt (Rodriguez-Tudela et al. 2008).

Vom Doktoranden wurde Microtiterplatten (greiner bio-one, Frickenhausen, Deutschland) mit den zu untersuchenden Antimykotika (Fluconazol, Voriconazol, Amphothericin B, Nystatin, Micafungin), den zugehörigen Medien (RPMI/ AM3) und Lösungsmitteln (steriles Wasser/ DMSO/ 75\% Methanol), unter Berücksichtigung des o. g. Protokolls hergestellt.

Die Medien wurden mit 0,4\% Glukose versetzt, um ein schnelleres Wachstum zu ermöglichen.

Zur Rekultivierung wurden die Candida-Stämme dem Stammerhaltungssystem entnommen. Durch einzelne Glasperlen aus den Kunststoffröhrchen konnten Zellen, welche zuvor eingefroren worden waren erneut angezüchtet werden. Dabei wurde darauf geachtet, dass die Organismen in den Kunststoffröhrchen nicht auftauten, um die Zellen nicht zu beschädigen. Die Organismen, die sich an der Oberfläche der Glaskugeln befanden wurden somit wiederholt auf Sabouraud-Nährböden ausgestrichen und im Brutschrank bei $37^{\circ} \mathrm{C}$ für 24 Stunden inkubiert. Um den Pilzkulturen eine langsame Regeneration nach tiefgekühlter Lagerung ermöglichen zu können, wurden die aufgetauten Stämme erneut überimpft. Somit wurde gewährleistet, dass die Kulturen zum Zeitpunkt der weiteren Verwendung nicht älter als 24 Stunden waren.

Am Folgetag wurden Stammsuspensionen hergestellt, indem einzelne Kolonien mit einer Impföse der Agarplatte entnommen und in steriler Kochsalzlösung gelöst wurden. Die Trübung der Lösung wurde mit einem Densimat-McFarland-Photometer bestimmt und auf einen Wert von 0,5 eingestellt (entspricht einer Zellmenge von $4 \mathrm{x}$ $10^{6}$ Zellen pro Millimeter [c/ml]).

Nach einem weiteren Verdünnungsschritt mit steriler Kochsalzlösung wurden die Zelllösungen unmittelbar auf die zuvor aufgetauten Mikrotiterplatten verteilt.

Auf einer Rundbodenplatte wurden jeweils parallel 8 Candida-Isolate aufgetragen (eine Platte mit 96 Positionen entspricht 8 Reihen und 12 Spalten). 
In den Positionen 1 bis 12 befanden sich der Reihe nach die Wachstumskontrolle (Trübungswert, der ein ungehindertes Wachstum darstellt) und die 11 Konzentrationsstufen der Verdünnungsreihen der verschiedenen Antimykotika. Der Trübungswert mit der Konzentrationsstufe „null“ diente ebenfalls als Wachstumskontrolle.

Die Mikrotiterplatten wurden im Vorfeld angelegt und bis zur Verwendung mit Plastikdeckeln abgedeckt, in sterilen Plastiktüten verpackt und bei ca. $-27{ }^{\circ} \mathrm{C}$ in einem keimarmen Gefrierschrank gelagert.

Hierzu wurden Stammlösungen der fünf Antimykotika, sowie im weiteren Verlauf Verdünnungsreihen angelegt, welche auf die einzelnen Spalten der Mikrotiterplatten entsprechend der Konzentrationsstufen verteilt wurden.

Nach einer Inkubationszeit von 17 Stunden (AM3) und 36 Stunden (RPMI) bei $37^{\circ} \mathrm{C}$ wurden alle Positionen der Platten sorgfältig mit einer Transferpipette, angefangen bei der niedrigsten Zellkonzentration, homogenisiert und anschließend photometrisch analysiert (Photometer der Firma Dynex Technologies).

Die unterschiedlichen Inkubationszeiten der beiden Medien wurden individuell durch Probemessungen angepasst.

Hierbei wurde in einem automatischen Ablesevorgang das Wachstum in jeder der einzelnen Positionen bestimmt.

Als Nullabgleich diente die zwölfte Position (Leerkontrolle).

Die Trübungswerte können stark durch punktuelles Wachstum oder Luftbläschen beeinflusst werden. Daher wurden vor jeder Messung die Suspensionen gründlich mittels einer Transferpipette durchmischt, sowie die Luftblasen entfernt.

Die sog. MHK (minimale Hemmkonzentration, engl:: MIC) ist definiert als die geringste Konzentration eines Antimykotikums, welche das Wachstum des Pilzes inhibiert. Sie gibt Aufschluss über die Wirksamkeit des verwendeten Medikamentes auf die zu untersuchende Spezies.

Die MHK wurde aus den ermittelten Daten für jede Spezies und jedes Antimykotikum ermittelt. Die MHK entspricht der Position in einer Reihe auf einer Titerplatte, bei der die Messung des Photometers den ersten, von den vorherigen abweichenden Trübungswerten aufweist. Hierbei waren üblicherweise die Werte einer Messung 
anfangs konstant niedrig, bis die Position erreicht wurde, an der die Konzentration des Antimykotikums ein erstes Wachstum der Hefen zugelassen hat. Jede Spezies wurde auf jedes der fünf Antimykotika jeweils dreimal getestet. Aus diesen drei Messwerten wurde anschließend ein Mittelwert gebildet.

Bei Messwerten, die sehr stark von den anderen ermittelten Werten abwichen oder eindeutig auf eine Kontamination hinwiesen (so auch Titerplatten die Zeichen einer Kontamination zeigten), wurde die Messung wiederholt um die Anzahl offensichtlich falscher Messwerte zu minimieren und möglichst exakte Aussagen über die Werte der MHK zu ermöglichen.

\subsubsection{Statistische Methoden}

Zur Berechnung univariater Signifikanzen wurden Vier- und Mehr-Felder-Tests mittels Pearson-Chi-Quadrat- (und zweiseitigem) Test angefertigt.

T-Tests wurden zur Berechnung von Signifikanzen der mikrobiologischen Befunde und des Alters herangezogen.

Logistische Regressionsmodelle dienten in einem zweiten Arbeitsschritt zur Testung mehrerer signifikanter Variablen (aus den einzelnen Testungen) und dem dazugehörigen mikrobiologischen Befund.

Die Berechnungen wurden mit der Software: STATISTICA, unter Anleitung von Herrn Dr. Jung aus der Abteilung Medizinische Statistik der Universität Göttingen, durchgeführt. 


\subsection{Material}

Die Materialien sowie Geräte sind den folgenden Tabellen 1-3 zu entnehmen.

Tabelle 1: Verbrauchsmaterialien

Verbrauchsmaterialien

96-Loch Rundboden-Mikrotiterplatten

Antibiotic Medium 3 (AM3)

Chrom-Agar Brilliance candida selective supplement

\section{Firma}

Greiner Bio-one GmbH, Frickenhausen

DIFCO, BD, New Jersey, USA

Oxoid, Hampshire, England

Chrom-Agar CM 1002 Brilliance Candida agar Oxoid, Hampshire, England base

Cryobank Stammhaltungssystem

Mast Diagnostica GmbH, Reinfeld

D-Glucose

ROTH, Karlsruhe

DMSO

SIGMA -Aldrich Chemie GmbH, Steinheim

Instamed - Medium RPMI 1640

Biochrom \& KG, Berlin

Malz-Agar

DIFCO, BD, New Jersey, USA

MOPS

SIGMA-Aldrich Chemie GmbH, Steinheim

Natriumchlorid

Merck \& Co, Inc., Whitehouse Station, USA

Parafilm M

American Can Company Greenwich CT

06830 / Dechiney

Parafilm

Plastic Packing, Chicago, IL, USA

Petrischalen

Greiner Bio-one GmbH, Frickenhausen

Pipettenspitzen - Biosphere Filter Tips No/Ref. Sarstedt, Nürnbrecht 70.762 .211

PS-Abdeckplatten

RPMI Medium

Sabouraud - 4\% Glucose -Agar

Sterile Wattetupfer
Greiner Bio-one GmbH, Frickenhausen

Biochrom AG, Berlin

Merck \& Co, Inc., Whitehouse Station, USA

Copan Diagnostics, Corona, CA, USA 
Tabelle 2: Antimykotika

\section{Antimykotika}

Amphothericin B

Fluconazol

Micafungin

Nystatin

Voriconazol

Tabelle 3: Geräte

\section{Geräte}

Arbeitsbank

Autoklav Provit 2200

Brutschrank Typ B 6120(I)

Brutschrank

Bunsenbrenner flammy S

Centrifuge 5415C

Densimat Mc-Farland-Photometer

Flachbodengläser Fiolax 80 x Ø17,75

x $0,55 \mathrm{~mm}$

Gefrierschrank

Glasflaschen Duran 100 ml, 200 ml,

$500 \mathrm{ml}, 2000 \mathrm{ml}$

Glas-Pasteurpipetten

Glaspipette $5 \mathrm{ml}, 10 \mathrm{ml}, 20 \mathrm{ml}$

Impfschlinge $1 \mu \mathrm{l}, 10 \mu \mathrm{l}$

Kühlschrank

Magnetrührer IKA -Combimag Reo

Autoflex III Massenspektrometer

MR x TC Revelation Photometer mit 630 nm Filter

Ph-Meter ph 526

Pipette model 5000 autoclavable

\section{Firma}

Sigma-Aldrich Chemie GmbH, Steinheim

Pfizer GmbH, Karlsruhe

Astellas Pharma, München

Sigma-Aldrich Chemie GmbH, Steinheim

Pfizer GmbH, Karlsruhe

\section{Firma/ Gerätbeschreibung}

BDK, Sonnenbühl-Genkingen

Holzner, Nußloch

Heraeus instruments, Hanau

Memmert elektronik, Schwabach

Schütt Labortechnik GmbH, Göttingen

Eppendorf AG, Hamburg

Bio-Mérieux, Marcy-l`Etoile, Frankeich

Rettberg, Göttingen

GFL, Burgwedel

Schott AG, Mainz

Brand, Wertheim

Brand, Wertheim

Sarstedt Aktiengesellschaft \&Co, Nümbrecht

Bosch, München

Ikamag RCT/ Janke \& Kunkel KG, Staufen/Breisgau

Bruker Daltonics, Billerica, USA

Dynex Technologies, Chantilly, VA, USA

Schütt Labortechnik GmbH, Göttingen

Nichiryo, Maryland Heights, USA 
Pipetten

Pipetus -Akku

Transferpette ${ }^{\circledR} 8$ x $20-200 \mu \mathrm{l}$

Vakuumpumpe

Vortex Genie 2

Waage BL 310
Eppendorf, Hamburg

Hirschmann Laborgeräte, Eberstadt

Brand, Wertheim

KNF Neuberger, Freiburg

Scientific Industry, Bohemia, NY, USA

Sartorius, Göttingen

\subsection{Ethikvotum}

Aus Sicht der Ethik- Kommission bestehen keine ethischen und rechtlichen Bedenken gegen die Durchführung dieses Forschungsvorhabens.

Die Studie wurde von der Ethikkommission der Medizinischen Fakultät Göttingen mit Votum zur Nummer 9/01/09 zustimmend bewertet. 


\section{Ergebnisse}

Der Abschnitt zu den Resultaten dieser Studie ist in drei Teile gegliedert. Zunächst werden die Ergebnisse des Befunderhebungsbogens (demografische Daten, anamnestische Angaben und klinische Befunde) dargestellt, gefolgt von den Ergebnissen der mikrobiologischen Diagnostik. In einem dritten statistischen Teil werden die Korrelationen zwischen klinischen Merkmalen und mikrobiologischen Befunden berechnet.

\subsection{Ergebnisse der Befunderhebungsbögen}

\subsubsection{Demografische Merkmale}

Im Verlauf des Untersuchungszeitraums von März 2009 bis Januar 2011 wurden Probanden von insgesamt drei stationären Pflegeeinrichtungen in die Studie aufgenommen. Von insgesamt 125 Probanden, welche von der Pflegeheimleitung ausgewählt wurden (s.o., Abschnitt 3.1.), gaben 80 Probanden (bzw. deren Betreuer) ihr Einverständnis zu der Teilnahme an der Studie.

\section{Pflegeheime}

Von den Pflegeheimen 1 (mit Schwerpunkt Demenzpflege) und 2 wurden insgesamt 50 Probanden um eine Teilnahme gebeten. In Pflegeheim 1 sind 37 und in Pflegeheim 2 insgesamt 30 Personen dieser Bitte nachgekommen.

Die Anzahl der Personen, die eine Teilnahme an der Studie verweigerten, beträgt für Pflegeheim 1: 13 (26\%) und für 2: 20 (40\%).

Um das Feld der Studienteilnehmer noch weiter zu vergrößern, wurden im Januar 2011 zusätzlich 25 weitere Personen aus einem dritten Pflegeheim gebeten, an der Studie zu partizipieren, wovon 13 sich bereiterklärten teilzunehmen und 12 Personen ablehnten (48\%).

Die Nicht-Teilnahme an der Studie war meist auf ein allgemeines Unwohlsein am Tag der Untersuchung zurückzuführen. Die meisten Probanden, die nicht an der Studie teilnehmen wollten, verweigerten die Teilnahme bereits zum Zeitpunkt der schriftlichen Aufklärung bzw. Projektvorstellung. 


\section{Altersverteilung}

Das durchschnittliche Alter der Teilnehmer betrug 83,5 Jahre, in Pflegeheim 1: 83,4 Jahre, in Pflegeheim 2: 85 Jahre und in Pflegeheim 3: 80 Jahre (Tabelle 4).

Das Pflegeheim 1 umfasst Personen im Alter von 65 bis 100 Jahren, Pflegeheim 2: 61 bis 99 Jahren und Pflegeheim 3: 49 bis 97 Jahren.

Die Probandengruppe im Alter zwischen 81 und 90 Jahren ist in Pflegeheim 1 und 2 am häufigsten vertreten.

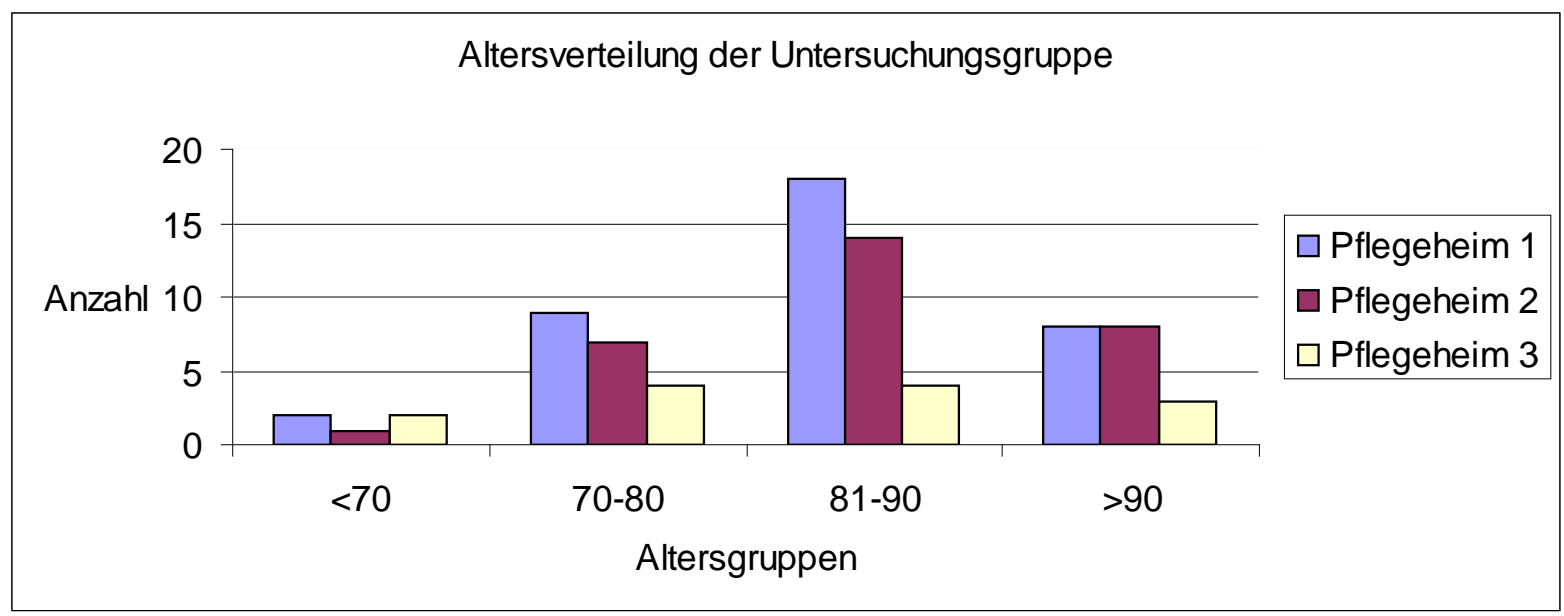

Abbildung 1: Altersverteilung der Untersuchungsgruppe

In dem dritten Pflegeheim ist die Anzahl der Probanden in der zweiten und dritten Altersgruppe identisch.

In allen drei Pflegeheimen weist die erste Altersgruppe (<70 Jahre) die geringste Probandenanzahl auf (Abbildung 1).

\section{Geschlechterverteilung}

Insgesamt nahmen 57 Frauen (71\%) und 23 Männer (29\%) an der Studie teil, wobei die Anzahl der teilnehmenden Frauen im ersten Pflegeheim mit 83\% am höchsten, in Pflegeheim 2 mit 67\% weniger stark und in Pflegeheim 3 mit 54\% am geringsten vertreten war. Die größere Anzahl weiblicher Teilnehmer liegt unter anderem an dem Bewohner-/ Bewohnerinnenverhältnis der Pflegeheime. 


\begin{tabular}{|c|c|c|c|c|c|c|c|c|}
\hline & \multicolumn{2}{|c|}{ Pflegeheim 1} & \multicolumn{2}{|c|}{ Pflegeheim 2} & \multicolumn{2}{|c|}{ Pflegeheim 3} & \multicolumn{2}{|c|}{ Gesamt } \\
\hline & $n$ & $(\%)$ & $n$ & $(\%)$ & $n$ & $(\%)$ & $n$ & $(\%)$ \\
\hline Anzahl & 37 & $(46,25)$ & 30 & $(37,5)$ & 13 & $(16,25)$ & 80 & (100) \\
\hline Männer & 7 & $(18,92)$ & 10 & $(33,33)$ & 6 & $(46,15)$ & 23 & $(28,75)$ \\
\hline Frauen & 30 & $(81,08)$ & 20 & $(66,67)$ & 7 & $(53,85)$ & 57 & $(71,25)$ \\
\hline$\varnothing$ Alter & 83,4 & & 85 & & 80. & & 83. & \\
\hline$\varnothing$ Alter d. Männer & 79,5 & & 84. & & 85,8 & & 83 & \\
\hline$\varnothing$ Alter d. Frauen & 84,3 & & 85 & & 75. & & 81 & \\
\hline
\end{tabular}

Tabelle 4: Verteilung der Probanden nach Alter und Geschlecht

Geschlechtsspezifische Altersverteilung

Bezogen auf die Altersgruppierung sind die weiblichen Studienteilnehmer der Pflegeheime 1 und 2, in der Altersgruppe von 81-90 Jahren und die weiblichen Probanden des dritten Pflegeheims in der Altersgruppe 70-80 Jahre am stärksten vertreten (Tabelle 5; Abbildung 2).

\begin{tabular}{|ll|llllllllll|}
\hline Alter & & \multicolumn{3}{l}{$<70$} & \multicolumn{3}{l}{$71-80$} & \multicolumn{3}{c}{$81-90$} & \multicolumn{2}{c|}{$>90$} & \multicolumn{3}{c|}{ Anzahl } \\
& & $\mathrm{n}$ & $(\%)$ & $\mathrm{n}$ & $(\%)$ & $\mathrm{n}$ & $(\%)$ & $\mathrm{n}$ & $(\%)$ & $\mathrm{n}$ & $(\%)$ \\
\hline Anzahl & & 5 & $(6,25)$ & 20 & $(25,00)$ & 36 & $(45,00)$ & 19 & $(23,75)$ & 80 & $(100)$ \\
männlich & & 1 & $(1,25)$ & 5 & $(6,25)$ & 12 & $(15,00)$ & 5 & $(6,25)$ & 23 & $(28,75)$ \\
weiblich & & 4 & $(5.00)$ & 15 & $(18,75)$ & 24 & $(30,00)$ & 14 & $(17,50)$ & 57 & $(71,25)$ \\
Pflegeheim1 & gesamt & 2 & $(2,50)$ & 9 & $(11,25)$ & 18 & $(22,50)$ & 8 & $(10,00)$ & 37 & $(46,25)$ \\
& männlich & 1 & $(1,25)$ & 2 & $(2,50)$ & 3 & $(3,75)$ & 1 & $(1,25)$ & 7 & $(8,75)$ \\
& weiblich & 1 & $(1,25)$ & 7 & $(8,75)$ & 15 & $(18,75)$ & 7 & $(8,75)$ & 30 & $(37,50)$ \\
Pflegeheim2 & qesamt & 1 & $(1,25)$ & 7 & $(8,75)$ & 14 & $(17,50)$ & 8 & $(10,00)$ & 30 & $(37,50)$ \\
& männlich & 0 & $(0)$ & 2 & $(2,50)$ & 6 & $(7,50)$ & 2 & $(2,50)$ & 10 & $(12,50)$ \\
& weiblich & 1 & $(1,25)$ & 5 & $(6,25)$ & 8 & $(10,00)$ & 6 & $(7,50)$ & 20 & $(25,00)$ \\
Pflegeheim3 & qesamt & 2 & $(2,50)$ & 4 & $(5,00)$ & 4 & $(5,00)$ & 3 & $(3,75)$ & 13 & $(16,25)$ \\
& männlich & 0 & $(0)$ & 1 & $(1,25)$ & 3 & $(3,75)$ & 2 & $(2,50)$ & 6 & $(7,50)$ \\
& weiblich & 2 & $(2,50)$ & 3 & $(3,75)$ & 1 & $(1,25)$ & 1 & $(1,25)$ & 7 & $(8,50)$ \\
\hline
\end{tabular}

Tabelle 5: Einteilung nach Altersgruppen 




Abbildung 2: Geschlechterverteilung nach Altersgruppen

\section{Kontrollgruppe}

Als Kontrollgruppe wurden Studierende der Medizin und Zahnmedizin der Universitätsmedizin Göttingen aus dem Sommersemester 2010 hinzugezogen. Insgesamt erklärten sich 47 Studierende dazu bereit, an der Studie teilzunehmen.

Das durchschnittliche Alter der Kontrollgruppe betrug 25 Jahre. Als Probanden standen 27 Frauen (57\%) und 20 Männer (43\%) zur Verfügung. Der Altersdurchschnitt der weiblichen Teilnehmer lag bei 24,6 Jahren und 25,9 Jahren bei den männlichen Teilnehmern (Tabelle 6).

\begin{tabular}{|l|lll|}
\hline \multicolumn{3}{l}{ Anzahl: $n(\%)$} & $\varnothing$ Alter \\
\hline Gesamt & 47 & $(100)$ & 25,15 \\
Männlich & 20 & $(42,55)$ & 25,90 \\
Weiblich & 27 & $(57,45)$ & 24,59 \\
\hline
\end{tabular}

Tabelle 6: Kontrollgruppe (Anzahl und durchschnittliches Alter)

\subsubsection{Klinische Merkmale}

Die Ergebnisse der Befunderhebungsbögen umfassen, wie bereits oben erwähnt, die Antworten der Studienteilnehmer bzw. in manchen Fällen die der pflegerischen Hilfskraft, sofern der Studienteilnehmer nicht für sich selbst sprechen konnte.

Die Fragen des Befunderhebungsbogens wurden kurz und verständlich gehalten. Dies spiegelte sich in der Befragung wider, in der die Fragen gut von den Probanden 
beantwortet werden konnten, so dass in den meisten Fällen das Pflegepersonal nicht hinzugezogen werden musste.

\section{Zugrundeliegende Diagnosen}

Ein Diabetes mellitus konnte bei 25 Probanden eruiert werden (Pflegeheim $1 \mathrm{n}=11$; Pflegeheim 2 n= 9; Pflegeheim 3 n= 5).

Auf die Frage nach der Ernährungsart (oral/ enteral/ parenteral) wurde diese in Pflegeheim 2 und 3 mit oral beantwortet. Lediglich in Pflegeheim 1 wurde die Ernährung einer Person enteral durchgeführt.

51 Probanden haben angegeben, jemals operiert worden zu sein (Pflegeheim $1 \mathrm{n}=$ 20; Pflegeheim 2 n= 20; Pflegeheim 3 n= 11).

2 Probanden aus Pflegeheim 2 wurden jemals einer Strahlentherapie unterzogen. Das übrige Probandenkollektiv hat die Frage nach einer Strahlentherapie verneint.

Die Frage nach einer jemals durchgeführten Chemotherapie wurde in einem Fall bejaht (Pflegeheim 3).

\section{Vormedikation}

Die Erfassung der Medikation ergab, dass bei 8 Probanden eine Behandlung mit Antibiotika erfolgte. In Pflegeheim 1 wurde ein Proband antibiotisch behandelt (Kanamycin). In Pflegeheim 2 wurden sieben Antibiotika unter den Medikamenten der Probanden verzeichnet (Clindamycin, Cotrimoxazol, Ciprofloxacin, Clarithromycin, Amoxicillin/ Clavulansäure, [Oral-] Cephalosporin). Die Medikation der Probanden aus Pflegeheim 3 beinhaltete keine Antibiotika.

Sieben Personen wurden zum Zeitpunkt der Untersuchung und Probenentnahme mit Immunsuppressiva behandelt. Eine Person aus Pflegeheim 1 wurde lokal mit Hydrocortison behandelt. In Pflegeheim 2 wurden vier, und in Pflegeheim 3 zwei Personen mit Prednison bzw. Prednisolon therapiert.

Eine Person in Pflegeheim 2 wurde mit einem Antimykotikum (Clotrimazol) behandelt (Tabelle 7). 


\begin{tabular}{|c|c|c|c|c|c|c|c|}
\hline & & \multicolumn{4}{|c|}{$\begin{array}{l}\text { Untersuchungsgruppe/ } \\
\text { Pflegeheime }\end{array}$} & \multirow[t]{2}{*}{$\begin{array}{l}\text { Kontroll- } \\
\text { gruppe }\end{array}$} & \multirow[t]{2}{*}{ Summe } \\
\hline & & 1 & 2 & 3 & Summe & & \\
\hline \multirow[t]{2}{*}{ Geschlecht } & männlich & 7 & 10 & 6 & 23 & 20 & 43 \\
\hline & weiblich & 30 & 20 & 7 & 57 & 27 & 84 \\
\hline \multirow[t]{2}{*}{ Diabetes mellitus } & nein & 26 & 21 & 8 & 55 & 0 & 55 \\
\hline & ja & 11 & 9 & 5 & 25 & 0 & 25 \\
\hline \multirow[t]{3}{*}{ Ernährung } & oral & 36 & 30 & 13 & 79 & 0 & 79 \\
\hline & enteral & 1 & 0 & 0 & 1 & 0 & 1 \\
\hline & parenteral & 0 & 0 & 0 & 0 & 0 & 0 \\
\hline \multirow[t]{3}{*}{ Operation } & $\mathrm{n} / \mathrm{a}$ & 0 & 0 & 0 & 0 & 47 & 47 \\
\hline & nein & 17 & 10 & 2 & 29 & 0 & 29 \\
\hline & ja & 20 & 20 & 11 & 51 & 0 & 51 \\
\hline \multirow[t]{3}{*}{ Bestrahlung } & $\mathrm{n} / \mathrm{a}$ & 0 & 0 & 0 & 0 & 47 & 47 \\
\hline & nein & 37 & 30 & 11 & 78 & 0 & 78 \\
\hline & ja & 0 & 0 & 2 & 2 & 0 & 2 \\
\hline \multirow[t]{3}{*}{ Chemotherapie } & $\mathrm{n} / \mathrm{a}$ & 0 & 0 & 0 & 0 & 47 & 47 \\
\hline & nein & 37 & 30 & 12 & 79 & 0 & 79 \\
\hline & ja & 0 & 0 & 1 & 1 & 0 & 1 \\
\hline \multirow[t]{3}{*}{ Andere Therapien } & $\mathrm{n} / \mathrm{a}$ & 0 & 0 & 0 & 0 & 47 & 47 \\
\hline & nein & 37 & 30 & 13 & 80 & 0 & 80 \\
\hline & ja & 0 & 0 & 0 & 0 & 0 & 0 \\
\hline \multirow[t]{3}{*}{ Antibiotika } & $\mathrm{n} / \mathrm{a}$ & 0 & 0 & 0 & 0 & 47 & 47 \\
\hline & nein & 36 & 23 & 13 & 72 & 0 & 72 \\
\hline & ja & 1 & 7 & 0 & 8 & 0 & 8 \\
\hline \multirow[t]{3}{*}{ Immunsupressiva } & $\mathrm{n} / \mathrm{a}$ & 0 & 0 & 0 & 0 & 47 & 47 \\
\hline & nein & 36 & 26 & 11 & 73 & 0 & 73 \\
\hline & ja & 1 & 4 & 2 & 7 & 0 & 7 \\
\hline \multirow[t]{3}{*}{ Antimykotika } & $\mathrm{n} / \mathrm{a}$ & 0 & 0 & 0 & 0 & 47 & 47 \\
\hline & nein & 37 & 29 & 13 & 79 & 0 & 79 \\
\hline & ja & 0 & 1 & 0 & 1 & 0 & 1 \\
\hline
\end{tabular}

Tabelle 7: Ergebnisse der Befunderhebungsbögen (1) Klinische Merkmale

\section{Pflegerische Aspekte}

Die Verteilung des ADL bzw. der Pflegestufe hat sich auf die Pflegeheime wie folgt verteilt: Unter Pflegestufe 0 wurde eine Person in Pflegeheim 2 geführt. Insgesamt 21 Personen waren zum Zeitpunkt der Untersuchung in die Pflegestufe 1 eingestuft worden (Pflegeheim $1 \mathrm{n}=5$; Pflegeheim $2 \mathrm{n=11}$; Pflegeheim $3 \mathrm{n=5}$ ). Die größte Probandenzahl, mit 38 Probanden (Pflegeheim $1 \mathrm{n}=23$; Pflegeheim $2 \mathrm{n}=7$; Pflegeheim $3 n=8$ ), wurde in der Gruppe der Pflegestufe 2 verzeichnet. Personen, die der höchsten Pflegestufe (3) zugeteilt waren, sind mit insgesamt 20 Personen vertreten, wobei 11 dem zweiten Pflegeheim und 9 dem ersten Pflegeheim zugeordnet werden konnten (Tabelle 8). 


\begin{tabular}{|c|c|c|c|c|c|}
\hline & \multicolumn{4}{|c|}{$\begin{array}{l}\text { Untersuchungsgruppe/ } \\
\text { Pflegeheime }\end{array}$} & \multirow[t]{2}{*}{ Kontrollgruppe } \\
\hline & 1 & 2 & 3 & Summe & \\
\hline ADL $0=$ selbstständige Personen & 0 & 1 & 0 & 1 & 0 \\
\hline 1= erheblich pflegebedürftige Person & 5 & 11 & 5 & 21 & 0 \\
\hline 2= schwer pflegebedürftige Person & 23 & 7 & 8 & 38 & 0 \\
\hline 3= schwerstpflegebedürftige Person & 9 & 11 & 0 & 20 & 0 \\
\hline
\end{tabular}

Tabelle 8: Ergebnisse der Befunderhebungsbögen (2) ADL

\section{Spezielle mundpflegerische Aspekte}

Die Mehrheit der befragten Probanden konnten der eigenständigen Mundhygiene nachkommen (Insgesamt: 43; Pflegeheim 1 n= 15; Pflegeheim 2 n= 16; Pflegeheim 3 $\mathrm{n}=12$ ). Bei 37 Probanden wurde die tägliche Mundhygiene durch das Pflegepersonal unterstützt bzw. durchgeführt (Pflegeheim 1 n= 22; Pflegeheim 2 n= 14; Pflegeheim 3 $\mathrm{n}=1)$.

75 Probanden gaben bezüglich der Mundhygiene an, dieser zwei- bis dreimal pro Tag nachzukommen (Pflegeheim 1 n= 37; Pflegeheim 2 n= 30; Pflegeheim $3 n=8$ ).

Lediglich 4 Personen gaben an, der Mundhygiene häufiger nachzukommen (Pflegeheim 3).

Die Mehrheit des Probandenkollektivs verwendete keine weiteren MundhygieneArtikel wie z.B.: Mundspüllösungen, Zahnseide, Mundduschen o.ä. (insgesamt: 65; Pflegeheim 1 n= 22; Pflegeheim 2 n= 23; Pflegeheim 3 n= 10).

14 Personen verwiesen auf zusätzliche Mundspüllösungen (Pflegeheim $1 \mathrm{n}=5$; Pflegeheim 2 n= 6; Pflegeheim 3 n= 3).

In einem Fall wurden weitere Mundhygiene-Artikel verwendet (Pflegeheim 2).

53 Probanden gaben an, eine Zahnprothese zu tragen bzw. zu besitzen. (Pflegeheim $1 \mathrm{n}=$ 23; Pflegeheim $2 \mathrm{n=22}$; Pflegeheim $3 \mathrm{n=}$ 8)

Die Erfassung der Prothesenhygiene zeigte, dass in vier Fällen keine Zahnersatzhygiene durchgeführt wurde (Pflegeheim $1 \mathrm{n}=0$; Pflegeheim $2 \mathrm{n}=3$; Pflegeheim $3 n=1$ ) und 5 Probanden der Reinigung des Zahnersatzes einmal am Tag nachkamen (Pflegeheim $1 \mathrm{n}=0$; Pflegeheim $2 \mathrm{n}=1$; Pflegeheim $3 \mathrm{n}=4$ ). Die Mehrheit gab an, ihre Zahnprothese zweimal täglich zu reinigen (insgesamt 41; Pflegeheim 1 n= 22; Pflegeheim $2 n=17$; Pflegeheim $3 n=2$ ). Im Vergleich der Pflegeheime war die Anzahl der Personen mit zweimaliger Mundhygiene pro Tag, in die ersten beiden Pflegeheime am häufigsten vertreten.

3 Personen (Pflegeheim $1 \mathrm{n}=1$; Pflegeheim $2 \mathrm{n}=1$; Pflegeheim $3 \mathrm{n}=1$ ) gaben an, dreimal täglich Ihre Prothese zu reinigen (Tabelle 9). 


\begin{tabular}{|c|c|c|c|c|c|c|c|}
\hline & & \multicolumn{4}{|c|}{$\begin{array}{l}\text { Untersuchungsgruppe/ } \\
\text { Pflegeheime }\end{array}$} & \multirow[t]{2}{*}{$\begin{array}{l}\text { Kontroll- } \\
\text { gruppe }\end{array}$} & \multirow[t]{2}{*}{ Summe } \\
\hline & & 1 & 2 & 3 & Summe & & \\
\hline \multirow[t]{3}{*}{ eigenst. $\mathrm{MH}$} & $\mathrm{n} / \mathrm{a}$ & 0 & 0 & 0 & 0 & 47 & 47 \\
\hline & nein & 22 & 14 & 1 & 37 & 0 & 37 \\
\hline & ja & 15 & 16 & 12 & 43 & 0 & 43 \\
\hline \multirow[t]{4}{*}{ Häufigkeit } & $0=$ keine Mundhygiene & 0 & 0 & 1 & 1 & 0 & 1 \\
\hline & $1=>3 \mathrm{mal} / \mathrm{Tag}$ & 0 & 0 & 0 & 0 & 0 & 0 \\
\hline & 2 = 2-3mal $/ \mathrm{Tag}$ & 37 & 30 & 8 & 75 & 43 & 118 \\
\hline & $3=<2 \mathrm{mal} / \mathrm{Tag}$ & 0 & 0 & 4 & 4 & 3 & 7 \\
\hline \multirow[t]{3}{*}{ sonst. MH- Artikel } & $0=$ keine $\mathrm{MH}$ Artikel & 32 & 23 & 10 & 65 & 0 & 65 \\
\hline & 1 = Mundspülung & 5 & 6 & 3 & 14 & 0 & 14 \\
\hline & 2 = Adstringentien & 0 & 1 & 0 & 1 & 0 & 1 \\
\hline \multirow[t]{2}{*}{ Prothese } & nein & 14 & 8 & 5 & 27 & 0 & 27 \\
\hline & ja & 23 & 22 & 8 & 53 & 0 & 53 \\
\hline \multirow[t]{4}{*}{ Prothesenhygiene } & $0 \mathrm{mal} / \mathrm{Tag}$ & 14 & 11 & 6 & 31 & 0 & 31 \\
\hline & $1 \mathrm{mal} / \mathrm{Tag}$ & 0 & 1 & 4 & 5 & 0 & 5 \\
\hline & $2 \mathrm{mal} / \mathrm{Tag}$ & 22 & 17 & 2 & 41 & 0 & 41 \\
\hline & $3 \mathrm{mal} / \mathrm{Tag}$ & 1 & 1 & 1 & 3 & 0 & 3 \\
\hline
\end{tabular}

Tabelle 9: Ergebnisse der Befunderhebungsbögen (3) Hygiene/ Prothetische Versorgung

\section{Verdauung}

3 Probanden berichteten über aktuelle Diarrhö (Pflegeheim $1 \mathrm{n}=1$; Pflegeheim $2 \mathrm{n}=$ 2; Pflegeheim $3 n=0$ ), eine Person vor 3-7 Tagen, vier Personen innerhalb der letzten 7-14 Tage (Pflegeheim $1 \mathrm{n}=2$; Pflegeheim $2 \mathrm{n}=1$; Pflegeheim $3 \mathrm{n=1}$ ) und vier Personen vor mehr als 14 Tagen (Pflegeheim $1 \mathrm{n}=1$; Pflegeheim $2 \mathrm{n}=3$; Pflegeheim $3 \mathrm{n}=0$ ) (Tabelle 10).

\section{Hauttypen}

Auf die Frage nach dem Hauttyp gab über die Hälfte der Untersuchungsgruppe an, die eigene Haut als trocken zu empfinden (insgesamt: 42; Pflegeheim $1 \mathrm{n}=16$; Pflegeheim $2 \mathrm{n}=16$; Pflegeheim $3 \mathrm{n}=10$ ). 37 Personen empfanden ihre Haut als normal (Pflegeheim $1 n=20$; Pflegeheim $2 n=14$; Pflegeheim $3 n=3$ ). Lediglich eine Person (Pflegeheim 1) bezeichnete ihren Hauttyp als fettig (Tabelle 10). 


\begin{tabular}{|c|c|c|c|c|c|c|c|}
\hline & & \multicolumn{4}{|c|}{ Untersuchungsgruppe/ Pflegeheime } & \multirow[t]{2}{*}{$\begin{array}{l}\text { Kontroll- } \\
\text { gruppe }\end{array}$} & \multirow[t]{2}{*}{ Summe } \\
\hline & & 1 & 2 & 3 & Summe & & \\
\hline \multirow[t]{6}{*}{ Verdauung } & $0=$ Nein & 32 & 24 & 12 & 68 & 0 & 68 \\
\hline & 1 = aktuell & 1 & 2 & 0 & 3 & 0 & 3 \\
\hline & $2=$ vor drei Tagen & 0 & 0 & 0 & 0 & 0 & 0 \\
\hline & $3=$ vor $3-7$ Tage & 1 & 0 & 0 & 1 & 0 & 1 \\
\hline & $4=$ vor $7-14$ Tage & 2 & 1 & 1 & 4 & 0 & 4 \\
\hline & $5=$ vor $>14$ Tage & 1 & 3 & 0 & 4 & 0 & 4 \\
\hline \multirow[t]{3}{*}{ Hauttyp } & $0=$ normal & 20 & 14 & 3 & 37 & 0 & 37 \\
\hline & 1 =trocken & 16 & 16 & 10 & 42 & 0 & 42 \\
\hline & $2=$ fettig & 1 & 0 & 0 & 1 & 0 & 1 \\
\hline
\end{tabular}

Tabelle 10: Ergebnisse der Befunderhebungsbögen (4) Verdauung / Hauttyp

\subsubsection{Klinische Symptome und Inspektion der Abstrichareale}

\section{Symptombelastung}

Die Anzahl klinischer Symptome zeigte sich vergleichsweise niedrig. In den Abstricharealen konnte bei der klinischen Inspektion nur wenige Auffälligkeiten beobachtet werden.

24 Probanden gaben an, dass sie entweder im Bereich der Mundhöhle, während des Sprech- oder Schluckvorganges oder auf der Haut unter Beschwerden litten (Pflegeheim $1 \mathrm{n}=15$; Pflegeheim $2 \mathrm{n=}$ 8; Pflegeheim $3 \mathrm{n}=1$ ).

Bei 18 Probanden äußerten sich diese Beschwerden in Form von einer verminderten Speichelsekretion (Pflegeheim $1 \mathrm{n}=12$; Pflegeheim $2 \mathrm{n}=6$; Pflegeheim $3 n=0$ ).

2 Personen berichteten über Zahnschmerzen (Pflegeheim $1 \mathrm{n}=0$; Pflegeheim $2 \mathrm{n}=2$; Pflegeheim $3 n=0$ ).

Eine Person (Pflegeheim 1) gab Mundgeruch/ Halitosis als subjektive Beschwerde an.

Über eine Dysphagie klagten 3 Probanden (Pflegeheim $1 \mathrm{n}=2$; Pflegeheim $2 \mathrm{n}=0$, Pflegeheim $3 \mathrm{n}=1$ ).

16 Probanden konnten nicht persönlich befragt werden (Pflegeheim $1 \mathrm{n}=12$; Pflegeheim $2 \mathrm{n}=4$; Pflegeheim $3 \mathrm{n}=0$ ). In diesen Fällen wurden die Fragen an das zuständige Pflegepersonal gerichtet (Tabelle 11). 


\begin{tabular}{|c|c|c|c|c|c|c|c|}
\hline & & \multicolumn{4}{|c|}{ Untersuchungsgruppe/ Pflegeheime } & \multirow[t]{2}{*}{ Kontrollgruppe } & \multirow[t]{2}{*}{ Summe } \\
\hline & & 1 & 2 & 3 & Summe & & \\
\hline \multirow[t]{2}{*}{ Symptomatik:Beschwerden } & nein & 22 & 22 & 12 & 56 & 0 & 56 \\
\hline & ja & 15 & 8 & 1 & 24 & 0 & 24 \\
\hline \multirow[t]{2}{*}{ Symptomatik: Xerostomie } & nein & 25 & 24 & 13 & 62 & 0 & 62 \\
\hline & ja & 12 & 6 & 0 & 18 & 0 & 18 \\
\hline \multirow[t]{2}{*}{ Symptomatik: Zahnschmerzen } & nein & 37 & 28 & 13 & 78 & 0 & 78 \\
\hline & ja & 0 & 2 & 0 & 2 & 0 & 2 \\
\hline \multirow[t]{2}{*}{ Symptomatik: Mundgeruch } & nein & 36 & 30 & 13 & 79 & 0 & 79 \\
\hline & ja & 1 & 0 & 0 & 1 & 0 & 1 \\
\hline \multirow[t]{2}{*}{ Symptomatik: unspez. Brennen } & nein & 37 & 30 & 13 & 80 & 0 & 80 \\
\hline & ja & 0 & 0 & 0 & 0 & 0 & 0 \\
\hline \multirow[t]{2}{*}{ Symptomatik: Dysgeusie } & nein & 37 & 30 & 13 & 80 & 0 & 80 \\
\hline & ja & 0 & 0 & 0 & 0 & 0 & 0 \\
\hline \multirow[t]{2}{*}{ Symptomatik: Dysphagie } & nein & 35 & 30 & 12 & 77 & 0 & 77 \\
\hline & ja & 2 & 0 & 1 & 3 & 0 & 3 \\
\hline \multirow[t]{2}{*}{ Symptomatik: Hautjucken } & nein & 37 & 30 & 13 & 80 & 0 & 80 \\
\hline & ja & 0 & 0 & 0 & 0 & 0 & 0 \\
\hline \multirow[t]{2}{*}{ Symptomatik: trockene Haut } & nein & 37 & 30 & 13 & 80 & 0 & 80 \\
\hline & ja & 0 & 0 & 0 & 0 & 0 & 0 \\
\hline \multirow[t]{2}{*}{ Symptomatik: Spannungsgefühl } & nein & 37 & 30 & 13 & 80 & 0 & 80 \\
\hline & ja & 0 & 0 & 0 & 0 & 0 & 0 \\
\hline \multirow[t]{2}{*}{ Patient nicht befragbar } & nein & 25 & 26 & 13 & 64 & 0 & 64 \\
\hline & ja & 12 & 4 & 0 & 16 & 0 & 16 \\
\hline
\end{tabular}

Tabelle 11: Ergebnisse der Befunderhebungsbögen (5) Symptombelastung

\section{Befunde der Mundhöhle}

Die Inspektion der Mundhöhle zeigte bei dem Großteil der Probandengruppe keine pathologischen Auffälligkeiten. Aufgrund der kurzen Beobachtungszeit und der mäßigen Beleuchtungsmöglichkeiten ohne weiteres Instrumentarium war jedoch nur eine oberflächliche, kurze Beurteilung möglich.

Bei 13 Personen konnte jedoch eine Schleimhautveränderung der Mundhöhle beobachtet werden (Pflegeheim $1 \mathrm{n}=2$, Pflegeheim $2 \mathrm{n}=7$; Pflegeheim $3 \mathrm{n}=4$ ).

8 Personen zeigten eine rötlich veränderte Mundschleimhaut (Pflegeheim $1 \mathrm{n}=1$; Pflegeheim 2 n= 5; Pflegeheim 3 n= 2). Weißliche, soortypische Beläge wurden bei 3 Personen beobachtet (Pflegeheim $1 \mathrm{n}=0$; Pflegeheim $2 \mathrm{n}=1$; Pflegeheim $3 \mathrm{n}=2$ ). Abweichende Beläge wurden in nur einem Fall beobachtet (Pflegeheim 2).

Weitere Schleimhautveränderungen wurden in einem Fall dokumentiert (Pflegeheim 1).

Zum Zeitpunkt der Untersuchungen wurden keine Ulzerationen, aphthöse Veränderungen oder akute Blutungen oder Petechien beobachtet. 
Die Speichelsekretion wurde von der Mehrheit der Untersuchungsgruppe als normal beschrieben. 6 Personen der 80 Befragten empfanden die Speichelsekretion als zu gering (Pflegeheim $1 \mathrm{n}=1$; Pflegeheim $2 \mathrm{n}=2$; Pflegeheim $3 \mathrm{n}=3$ ) und lediglich 2 Personen (Pflegeheim 2) berichteten über eine subjektiv erhöhte Speichelproduktion (Tabelle 12).

\begin{tabular}{|c|c|c|c|c|c|c|c|}
\hline & & \multicolumn{4}{|c|}{$\begin{array}{l}\text { Untersuchungsgruppe/ } \\
\text { Pflegeheime }\end{array}$} & \multirow[t]{2}{*}{$\begin{array}{l}\text { Kontroll- } \\
\text { gruppe }\end{array}$} & \multirow[t]{2}{*}{ Summe } \\
\hline & & 1 & 2 & 3 & Summe & & \\
\hline \multirow[t]{2}{*}{ klinisch auffälliger Befund der Mundhöhle } & nein & 35 & 23 & 9 & 67 & 46 & 113 \\
\hline & ja & 2 & 7 & 4 & 13 & 0 & 13 \\
\hline \multirow[t]{2}{*}{ Befund Mundhöhle: gerötet } & nein & 36 & 25 & 11 & 72 & 46 & 118 \\
\hline & ja & 1 & 5 & 2 & 8 & 0 & 8 \\
\hline \multirow[t]{2}{*}{ Befund Mundhöhle: geschwollen } & nein & 37 & 30 & 13 & 80 & 46 & 126 \\
\hline & ja & 0 & 0 & 0 & 0 & 0 & 0 \\
\hline \multirow[t]{2}{*}{ Befund Mundhöhle: soortypische Beläge } & nein & 37 & 29 & 11 & 77 & 46 & 123 \\
\hline & ja & 0 & 1 & 2 & 3 & 0 & 3 \\
\hline \multirow[t]{2}{*}{ Befund Mundhöhle: andere Beläge } & nein & 37 & 29 & 13 & 79 & 46 & 125 \\
\hline & ja & 0 & 1 & 0 & 1 & 0 & 1 \\
\hline \multirow[t]{2}{*}{ Befund Mundhöhle: Schleimhautveränderungen } & nein & 36 & 30 & 13 & 79 & 46 & 125 \\
\hline & ja & 1 & 0 & 0 & 1 & 0 & 1 \\
\hline \multirow[t]{2}{*}{ Befund Mundhöhle: Ulcus } & nein & 37 & 30 & 13 & 80 & 46 & 126 \\
\hline & ja & 0 & 0 & 0 & 0 & 0 & 0 \\
\hline \multirow[t]{2}{*}{ Befund Mundhöhle: Blutungen } & nein & 37 & 30 & 13 & 80 & 46 & 126 \\
\hline & ja & 0 & 0 & 0 & 0 & 0 & 0 \\
\hline \multirow[t]{2}{*}{ Befund Mundhöhle: Aphten } & nein & 37 & 30 & 13 & 80 & 46 & 126 \\
\hline & ja & 0 & 0 & 0 & 0 & 0 & 0 \\
\hline \multirow[t]{2}{*}{ Befund Mundhöhle: nicht beurteilbar/ Patient intubiert } & nein & 37 & 30 & 13 & 80 & 46 & 126 \\
\hline & ja & 0 & 0 & 0 & 0 & 0 & 0 \\
\hline \multirow[t]{3}{*}{ Speichelsekretion } & $0=$ normal & 36 & 26 & 10 & 72 & 0 & 72 \\
\hline & $1=$ reduziert & 1 & 2 & 3 & 6 & 0 & 6 \\
\hline & $2=$ vermehrt & 0 & 2 & 0 & 2 & 0 & 2 \\
\hline
\end{tabular}

Tabelle 12: Ergebnisse der Befunderhebungsbögen (6) Befund Mundhöhle

\section{Befunde der Haut}

Bei der Inspektion der jeweiligen Abstrichareale konnten bei den meisten Patienten keine Auffälligkeiten festgestellt werden (insgesamt: 68; Pflegeheim $1 \mathrm{n}=32$; Pflegeheim $2 \mathrm{n}=26$; Pflegeheim $3 \mathrm{n}=10$ ). Bei 11 Probanden waren rötlichentzündliche Veränderungen in einem der Abstrichareale zu erkennen (Pflegeheim 1 $\mathrm{n}=5$; Pflegeheim $2 \mathrm{n}=4$; Pflegeheim $3 \mathrm{n}=2$ ). Eine Person zeigte schuppige Veränderungen an einem der Abstrichareale (Pflegeheim 3). 
Auffälligkeiten außerhalb des Abstrichareals wurden bei insgesamt 10 Probanden festgestellt. 9 Personen zeigten gerötete Hautregionen um die Abstrichareale, wie z.B. in der Leistenfalte (Tabelle 13).

\begin{tabular}{|c|c|c|c|c|c|c|c|}
\hline & & \multicolumn{4}{|c|}{$\begin{array}{l}\text { Untersuchungsgruppe/ } \\
\text { Pflegeheime }\end{array}$} & \multirow[t]{2}{*}{$\begin{array}{l}\text { Kontroll } \\
\text { gruppe }\end{array}$} & \multirow[t]{2}{*}{ Summe } \\
\hline & & 1 & 2 & 3 & Summe & & \\
\hline \multirow[t]{7}{*}{ Auffälligkeiten (Abstrichareal) } & $0=$ keine & 32 & 26 & 10 & 68 & 0 & 68 \\
\hline & 1 = gerötet & 5 & 4 & 2 & 11 & 0 & 11 \\
\hline & 2 = geschwollen & 0 & 0 & 0 & 0 & 0 & 0 \\
\hline & $3=$ offene Wunde & 0 & 0 & 1 & 1 & 0 & 1 \\
\hline & 4 = Schuppen & 0 & 0 & 0 & 0 & 0 & 0 \\
\hline & $5=$ nicht beurteilbar & 0 & 0 & 0 & 0 & 0 & 0 \\
\hline & $\mathrm{n} / \mathrm{a}$ & 0 & 0 & 0 & 0 & 47 & 47 \\
\hline \multirow[t]{3}{*}{ Auffälligkeiten (Abstrichareal) } & Nein & 32 & 26 & 10 & 68 & 0 & 68 \\
\hline & ja & 5 & 4 & 3 & 12 & 0 & 12 \\
\hline & $\mathrm{n} / \mathrm{a}$ & 0 & 0 & 0 & 0 & 47 & 47 \\
\hline \multirow[t]{3}{*}{ sonst. Auff.: ja/nein } & nein & 34 & 27 & 9 & 70 & 0 & 70 \\
\hline & ja & 3 & 3 & 4 & 10 & 0 & 10 \\
\hline & $\mathrm{n} / \mathrm{a}$ & 0 & 0 & 0 & 0 & 47 & 47 \\
\hline \multirow[t]{3}{*}{ sonst. Auff.: keine } & nein & 3 & 3 & 4 & 10 & 0 & 10 \\
\hline & ja & 34 & 27 & 9 & 70 & 0 & 70 \\
\hline & $\mathrm{n} / \mathrm{a}$ & 0 & 0 & 0 & 0 & 47 & 47 \\
\hline \multirow[t]{3}{*}{ sonst. Auff.: gerötet } & nein & 34 & 27 & 10 & 71 & 0 & 71 \\
\hline & ja & 3 & 3 & 3 & 9 & 0 & 9 \\
\hline & $\mathrm{n} / \mathrm{a}$ & 0 & 0 & 0 & 0 & 47 & 47 \\
\hline \multirow[t]{3}{*}{ sonst. Auff.: geschwollen } & nein & 37 & 30 & 13 & 80 & 0 & 80 \\
\hline & ja & 0 & 0 & 0 & 0 & 0 & 0 \\
\hline & $\mathrm{n} / \mathrm{a}$ & 0 & 0 & 0 & 0 & 47 & 47 \\
\hline \multirow[t]{3}{*}{ sonst. Auff.: offene Wunde } & nein & 37 & 30 & 13 & 80 & 0 & 80 \\
\hline & ja & 0 & 0 & 0 & 0 & 0 & 0 \\
\hline & $\mathrm{n} / \mathrm{a}$ & 0 & 0 & 0 & 0 & 47 & 47 \\
\hline
\end{tabular}

Tabelle 13: Ergebnisse der Befunderhebungsbögen (7) Hautbefunde (1)

Eine Person zeigte auch in anderen Arealen schuppige Hautveränderungen (Pflegeheim 3).

In allen drei Pflegeheimen zeigte sich bezüglich der Fußnagelpflege ein ähnliches Bild. Die große Mehrheit der Probanden nahm die Hilfe einer professionellen Fußpflege in Anspruch. Bei 42 Probanden wurde diese ca. alle 6 Wochen in Anspruch genommen (Pflegeheim $1 \mathrm{n}=33$; Pflegeheim $2 \mathrm{n=1}$; Pflegeheim $3 \mathrm{n=}$ ). Lediglich eine Person aus jedem der drei Pflegeheime kümmerte sich eigenständig um die Fußhygiene und Nagelpflege. 
Insgesamt 36 Personen antworteten auf die Frage nach der Häufigkeit mit ca. vier Wochen (Pflegeheim 1 n= 4; Pflegeheim 2 n= 28; Pflegeheim $3 n=4$ ).

Nur 2 Personen gaben kürzere Intervalle von 2 Wochen an (Pflegeheim $2 \mathrm{n}=1$; Pflegeheim $3 \mathrm{n}=1$ ).

Bei 60 der 80 Probanden konnten keine Auffälligkeiten im Bereich des Zehenzwischenraumes festgestellt werden.

Bei 16 der 20 Personen, die Auffälligkeiten dieses Abstrichareals zeigten, machten die Nägel, die Zehen oder deren Zwischenräume oberflächlich einen ungepflegten Eindruck. Bei 3 Probanden konnten Entzündungszeichen in Form von Rötungen beobachtet werden. In einem Fall war der Zehennagel deutlich verfärbt (Tabelle 14).

\begin{tabular}{|c|c|c|c|c|c|c|c|}
\hline & & \multicolumn{4}{|c|}{$\begin{array}{l}\text { Untersuchungsgruppe/ } \\
\text { Pflegeheime }\end{array}$} & \multirow[t]{2}{*}{$\begin{array}{l}\text { Kontroll } \\
\text { gruppe }\end{array}$} & \multirow[t]{2}{*}{ Summe } \\
\hline & & 1 & 2 & 3 & Summe & & \\
\hline \multirow[t]{3}{*}{ sonst. Auff.: Schuppen } & nein & 37 & 30 & 12 & 79 & 0 & 79 \\
\hline & ja & 0 & 0 & 1 & 1 & 0 & 1 \\
\hline & $\mathrm{n} / \mathrm{a}$ & 0 & 0 & 0 & 0 & 47 & 47 \\
\hline \multirow[t]{3}{*}{ sonst. Auff.: nicht beurteilbar } & nein & 37 & 30 & 13 & 80 & 0 & 80 \\
\hline & ja & 0 & 0 & 0 & 0 & 0 & 0 \\
\hline & $\mathrm{n} / \mathrm{a}$ & 0 & 0 & 0 & 0 & 47 & 47 \\
\hline \multirow[t]{3}{*}{ sonst. Auff.: massive Ödeme } & nein & 37 & 30 & 13 & 80 & 0 & 80 \\
\hline & ja & 0 & 0 & 0 & 0 & 0 & 0 \\
\hline & $\mathrm{n} / \mathrm{a}$ & 0 & 0 & 0 & 0 & 47 & 47 \\
\hline \multirow[t]{3}{*}{ Fußnagelpflege (eigenständig) } & ja & 1 & 1 & 1 & 3 & 0 & 3 \\
\hline & nein & 36 & 29 & 12 & 77 & 0 & 77 \\
\hline & $\mathrm{n} / \mathrm{a}$ & 0 & 0 & 0 & 0 & 47 & 47 \\
\hline \multirow[t]{4}{*}{ Fußnagelpflege (Häufigkeit) } & $0,5=$ ca. alle 6 Wochen & 33 & 1 & 8 & 42 & 0 & 42 \\
\hline & 1 =ca. $1 \mathrm{mal} /$ Monat & 4 & 28 & 4 & 36 & 0 & 36 \\
\hline & $2=>1 \mathrm{mal} /$ Monat & 0 & 1 & 1 & 2 & 0 & 2 \\
\hline & $\mathrm{n} / \mathrm{a}$ & 0 & 0 & 0 & 0 & 47 & 47 \\
\hline \multirow[t]{3}{*}{ Fußnägelstatus: auffällig } & nein & 31 & 21 & 8 & 60 & 46 & 106 \\
\hline & ja & 6 & 9 & 5 & 20 & 0 & 20 \\
\hline & $\mathrm{n} / \mathrm{a}$ & 0 & 0 & 0 & 0 & 47 & 47 \\
\hline \multirow[t]{3}{*}{ Fußnägelstatus: ungepflegt } & nein & 31 & 22 & 11 & 64 & 46 & 110 \\
\hline & ja & 6 & 8 & 2 & 16 & 0 & 16 \\
\hline & $\mathrm{n} / \mathrm{a}$ & 0 & 0 & 0 & 0 & 47 & 47 \\
\hline \multirow[t]{3}{*}{ Fußnägelstatus: Entzündungszeichen } & nein & 37 & 30 & 10 & 77 & 46 & 123 \\
\hline & ja & 0 & 0 & 3 & 3 & 0 & 3 \\
\hline & $\mathrm{n} / \mathrm{a}$ & 0 & 0 & 0 & 0 & 47 & 47 \\
\hline \multirow[t]{3}{*}{ Fußnägelstatus: verfärbt } & nein & 37 & 29 & 13 & 79 & 46 & 125 \\
\hline & ja & 0 & 1 & 0 & 1 & 0 & 1 \\
\hline & $\mathrm{n} / \mathrm{a}$ & 0 & 0 & 0 & 0 & 47 & 47 \\
\hline
\end{tabular}

Tabelle 14: Ergebnisse der Befunderhebungsbögen (7) Hautbefunde (2)

Die Ergebnisse der Auswertung der Befunderhebungsbögen der Kontrollgruppe sind ebenfalls den Tabellen 7-14 zu entnehmen. 


\subsection{Ergebnisse mikrobiologischer Untersuchungen}

\subsubsection{Häufigkeit der mykologischen Befunde}

Mit Blick auf die oben beschriebenen Voruntersuchungen fokussierten sich die mikrobiologischen Untersuchungen auf die Diagnostik und Resistenzbestimmung von Candidabesiedlungen.

\section{Gesamtprävalenzen}

Bei über 70\% der Probanden konnten Pilze nachgewiesen werden. Am häufigsten wurden Hefen in oralen Proben nachgewiesen.

Insgesamt konnten bei 61 von 80 Personen (76\%) der Untersuchungsgruppe eine Besiedlung mit Candida-Spezies festgestellt werden.

Die größte Probandenanzahl mit positivem Candida-Befund unter den Probanden der jeweiligen Gruppen zeigen Probanden aus dem Pflegeheim 2 und Pflegeheim 3 mit 77\% (23 von 30 Personen in Pflegeheim 2 und 10 von 13 Personen in Pflegeheim 3). In Pflegeheim 1 (mit schwerpunktmäßiger Betreuung von Demenzpatienten) wurden 76\% (28 von 37 Personen) der Probanden mit positivem Candida-Befund ermittelt (Tabelle 15).

\begin{tabular}{|lcccccccr|}
\hline Probanden mit Candida & \multicolumn{2}{c}{ Pflegeheim 1 } & \multicolumn{2}{c|}{ Pflegeheim 2 } & \multicolumn{2}{c|}{ Pflegeheim 3 } & \multicolumn{2}{c|}{ Summe } \\
& $\mathrm{n}=37$ & $(\%)$ & $\mathrm{n}=30$ & $(\%)$ & $\mathrm{n}=13$ & $(\%)$ & $\mathrm{n}=80$ & $(\%)$ \\
\hline Gesamt & 28 & $(35,00)$ & 23 & $(28,75)$ & 10 & $(12,50)$ & 61 & $(76,00)$ \\
Männlich & 6 & $(7,50)$ & 9 & $(11,25)$ & 4 & $(5,00)$ & 19 & $(23,75)$ \\
Weiblich & 22 & $(27,50)$ & 14 & $(17,50)$ & 6 & $(7,50)$ & 42 & $(52,50)$ \\
\hline
\end{tabular}

Tabelle 15: Candidabesiedlung aller Probanden der Untersuchungsgruppe (80)

\section{Abstriche der Mundschleimhaut}

Die mit Abstand häufigste Anzahl der Candidanachweise wurde in allen drei Pflegeheimen im oralen Abstrichareal gefunden (Pflegeheim 1: 100\% der oralen Proben; Pflegeheim 2: 96\% und Pflegeheim 3: 80\%). So stammte die Mehrzahl der positiven Abstriche aus dem oralen Bereich. Ebenso war die Mehrheit der oralen Abstriche positiv. 
weitere Abstrichorte

An der Leistenfalte wurde in den Pflegeheimen 1 und 3 keine Candida isoliert. Lediglich in Pflegeheim 2 hatten 9\% der Probanden nachweislich einen positiven Abstrich auf der Haut der Leistenfalte.

Am dritten Abstrichort (Zehenzwischenräume) stellte sich in den Pflegeheimen 2 und 3 eine Besiedlung von 9\% und 30\% heraus während in Pflegeheim 1 erneut keine Anzeichen von Candida nachgewiesen werden konnten. (Abbildung 3)

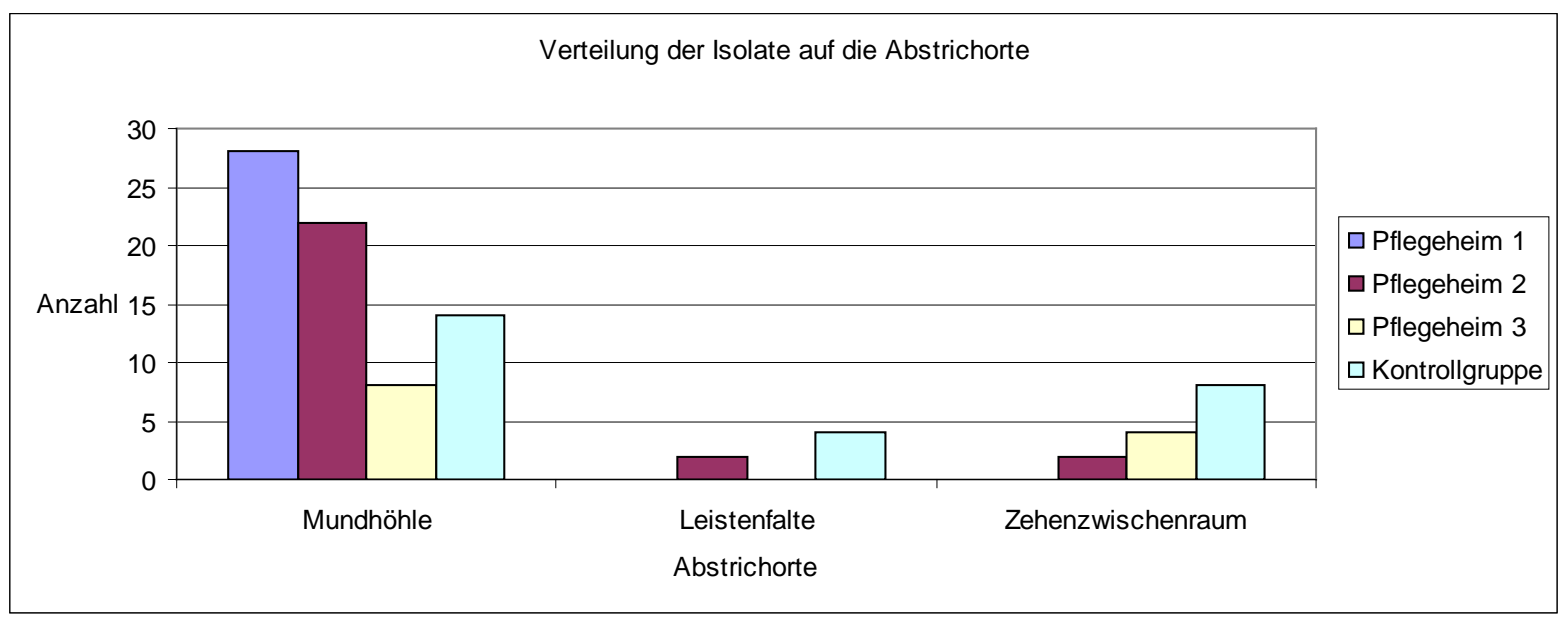

Abbildung 3: Verteilung der Isolate auf die Abstrichorte

\section{Kontrollgruppe}

Innerhalb der Kontrollgruppe konnten bei 22 von 47 Probanden Candidabesiedlungen nachgewiesen werden (47\%). In den Proben von 25 Teilnehmern wurden keine Anzeichen einer Pilzbesiedlung gefunden (53\%).

In 14 oralen Abstrichen wurden Hefenachweise gefunden. In 8 Fällen konnten Candida-Spezies in den Abstrichen des Zehenzwischenraumes und in 4 Fällen in den Abstrichen der Leistenfalte festgestellt werden. 


\subsubsection{Vergleich Untersuchungs- und Kontrollgruppe}

\section{Anzahl der Probanden mit Candida}

In den Abstrichen der insgesamt 80 Probanden der Untersuchungsgruppe (alle drei Pflegeheime) konnten bei 61 Probanden Hefen nachgewiesen werden (76\%). In der Kontrollgruppe (47 Probanden) waren es 22 Probanden (47\%).

\section{Anzahl und Differenzierung der positiven Pilzbefunde („Isolate“)}

Insgesamt wurden 103 Hefen isoliert („Isolate“: Hefenachweise der Mundschleimhaut- und Hautabstriche zusammengerechnet), davon 73 Isolate (71\%) in der Untersuchungsgruppe (Pflegeheim 1 mit 27\%, Pflegeheim 2 mit 30\% und Pflegeheim 3 mit 14\%) und 30 (29\%) in der Kontrollgruppe.

Die insgesamt 103 Isolate verteilen sich auf beide Gruppen, bezogen auf den Abstrichort wie folgt:

Die meisten Isolate wurden in den Mundschleimhautabstrichen (Abstrichort 1) gefunden.

Der zweite Abstrichort, die Leistenfalte, zeigt in der Untersuchungs-, wie auch der Kontrollgruppe die geringste Anzahl an Isolaten (Tabelle 16).

\begin{tabular}{|c|c|c|c|c|}
\hline & Abstrichort 1 & Abstrichort 2 & Abstrichort 3 & Summe \\
\hline & $(\%)$ & $(\%)$ & $(\%)$ & (\%) \\
\hline uchungsgruppe & $65(63,11)$ & $2(1,94)$ & $6 \quad(5,83)$ & $73(71,00)$ \\
\hline Kontrollgruppe & $15(14,56)$ & $5(4,85)$ & $10(9,71)$ & $30(29,00)$ \\
\hline Summe & $80(77,67)$ & $7(6,80)$ & $16(15,53)$ & $103(100)$ \\
\hline
\end{tabular}

Tabelle 16: Anzahl Spezies verteilt auf beide Gruppen

In der Untersuchungsgruppe wurden bei $89 \%$ der oralen Isolate eine Candidabesiedlung nachgewiesen, in den oralen Isolaten der Kontrollgruppe 50\% (Tabelle 17). Auf die Zahl der Probanden der beiden Gruppen zurück gerechnet, bedeutet dies, dass in der Untersuchungsgruppe auf jeden Probanden 91\% (73 Isolate auf 80 Probanden) Isolate entfallen und in der Kontrollgruppe auf jeden Probanden immerhin 64\% Isolate (30 Isolate auf 47 Probanden).

Die Anzahl der Candidaisolate war hingegen in den Abstrichbereichen Leistenfalte und Zehenzwischenraum in der Kontrollgruppe höher als in der Untersuchungsgruppe. In der Leistenfalte und dem Zehenzwischenraum wurden in den Untersuchungsgruppen $3 \%$ und $8 \%$ positive Candidanachweise des jeweiligen 
Abstrichortes gefunden. In der Kontrollgruppe wurden 17\% und 33\% der Isolate dieser Abstrichregionen positiv auf Candida getestet.

\begin{tabular}{|l|llll|}
\hline & \multicolumn{2}{c}{ Untersuchungsgruppe } & \multicolumn{2}{c|}{ Kontrollgruppe } \\
& $\mathrm{n}$ & $(\%)$ & $\mathrm{n}$ & $(\%)$ \\
\hline Abstrichort 1 & 65 & $(89,00)$ & 15 & $(50,00)$ \\
Abstrichort 2 & 2 & $(3,00)$ & 5 & $(17,00)$ \\
Abstrichort 3 & 6 & $(8,00)$ & 10 & $(33,00)$ \\
Summe & 73 & $(100)$ & 30 & $(100)$ \\
\hline
\end{tabular}

Tabelle 17: Prozentualer Vergleich beider Gruppen bezogen auf Abstrichort

\section{Geschlechtervergleich}

In der Untersuchungs- und Kontrollgruppe wurden bei den weiblichen Teilnehmern insgesamt 62 Candidaisolate gefunden. Bei den männlichen Teilnehmern waren es insgesamt 39. Es wurde bei einigen Probanden mehr als eine Spezies in einem Abstrich gefunden.

Es konnte kein Zusammenhang zwischen dem Geschlecht und der Spezies aufgezeigt werden. Insgesamt konnten bei Frauen mehr Pilzbesiedlungen nachgewiesen werden. Jedoch war die Anzahl der weiblichen Teilnehmer der Anzahl männlicher Teilnehmer überlegen (Abbildung 4). Die absoluten Zahlen der positiven Pilzbefunde waren daher bei Frauen höher. Prozentual wurden positive mikrobiologische Befunde jedoch häufiger bei Männern nachgewiesen.

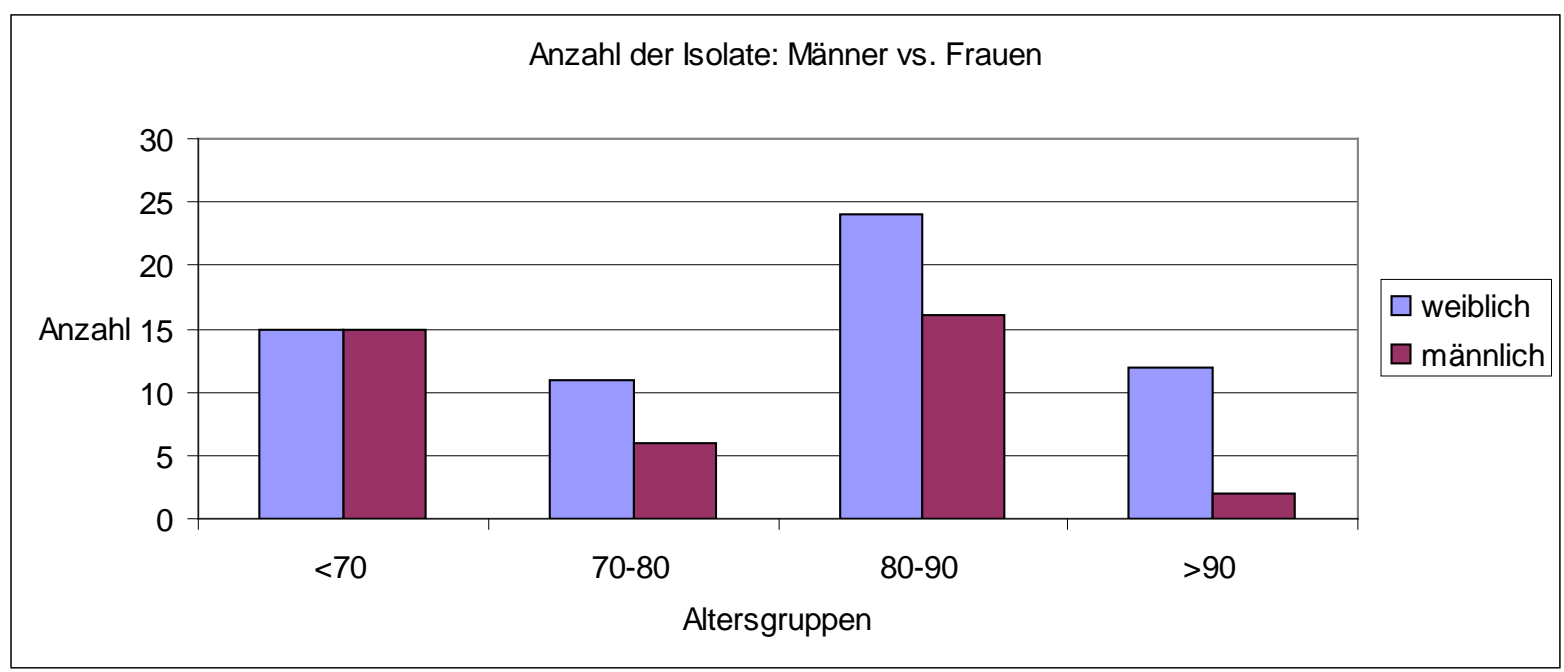

Abbildung 4: Absolute Anzahl der Isolate aus Untersuchungs- und Kontrollgruppe verteilt auf die Geschlechter 
Die größere Anzahl der Isolate auf Seiten der weiblichen Studienteilnehmer beruhte auf der insgesamt stärkeren Beteiligung weiblicher Probanden an der Studie.

Vergleicht man die Anzahl der gefundenen Isolate, geschlechtsspezifisch aufgeteilt (62 in der Gruppe der Frauen, 39 in der Gruppe der Männer) (die Gesamtzahl der Isolate beträgt hier 101 und nicht 103 da in zwei Isolaten zweier Probanden der Kontrollgruppe jeweils 2 Spezies isoliert wurden), mit der Anzahl der männlichen und weiblichen Probanden in den jeweiligen Altersgruppen, so zeigt sich, dass im Alter von $<70-90$ prozentual mehr Männer positive Candidabefunde aufweisen. (Abbildung 5)

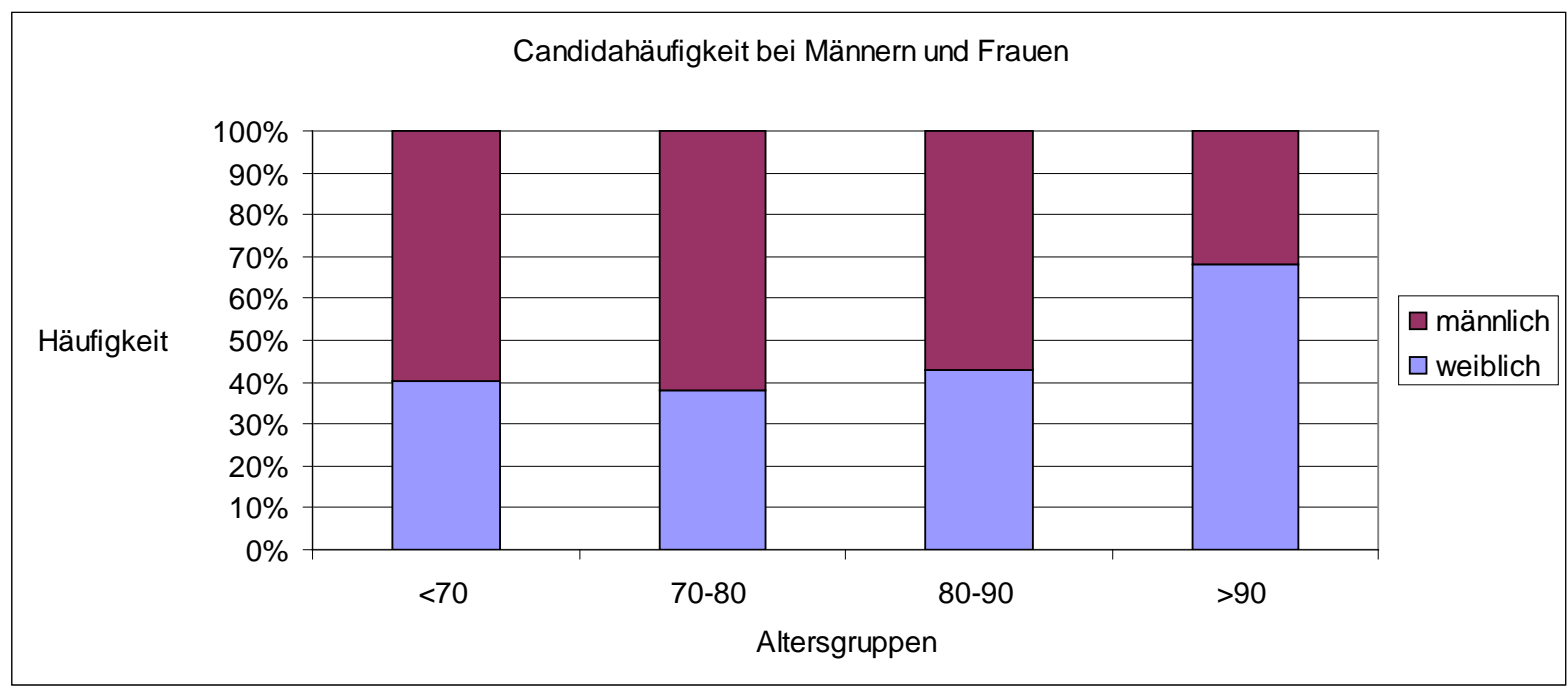

Abbildung 5: Prozentualer Vergleich der Isolate beider Geschlechter bezogen auf die männlichen/weiblichen Probanden der betreffenden Altersgruppe

In der Gruppe der über 90-Jährigen zeigt sich eine umgekehrte Verteilung. In dieser Gruppe haben Frauen prozentual einen größeren Anteil an mikrobiologischen Candidanachweisen als die männlichen Probanden der gleichen Altersgruppe.

Ursächlich für die Verteilung waren Mischkulturen (mehr als eine Spezies pro Abstrich). In der Gruppe 70-80-jähriger Männer wurden 6 Hefen bei nur 5 Probanden isoliert. In der Gruppe der 80-90-Jährigen waren es 16 Isolate bei 12 Probanden. Die absolute Anzahl der Isolate war bei den weiblichen Teilnehmern höher (Abbildung 4), jedoch verteilten sich die Isolate auf mehrere Proben verschiedener Probanden. 


\section{Geschlechtsspezifische Ergebnisse der Untersuchungsgruppe}

24\% der männlichen Teilnehmer und 53\% der weiblichen Studienteilnehmer der Untersuchungsgruppe sind in einem der drei Proben positiv auf Candida getestet worden.

Im Vergleich der Geschlechter ist die Anzahl der Candidaisolate bei Männern prozentual höher als bei dem weiblichen Anteil der Probanden. 19 der insgesamt 23 männlichen Teilnehmer zeigten nachweislich eine Candidabesiedlung in einer oder mehr Proben der drei Abstrichareale (83\% der Männer), wohingegen nur 74\% der weiblichen Teilnehmer eine Pilzbesiedlung aufwiesen (42 der 57 weiblichen Probanden).

Von den auf Candida positiv getesteten Probanden waren 69\% weiblich und 31\% männlich. Die absolute Anzahl weiblicher Probanden war insgesamt jedoch höher.

Einen prozentualen Vergleich der männlichen und weiblichen Probanden der drei Pflegeheime zeigt den deutlichen Unterschied in der Geschlechterverteilung bezogen auf alle Probanden des Pflegeheims, der Untersuchungsgruppe sowie alle Probanden der Untersuchungsgruppe mit Pilzbefall (Tabelle 18).

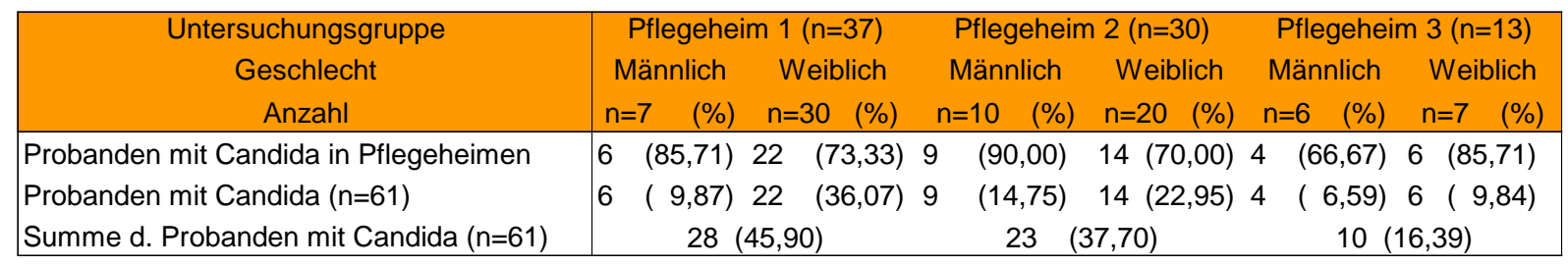

Tabelle 18: Verteilung der Candidabesiedlung innerhalb der Gruppen

\section{Geschlechtsspezifische Ergebnisse der Kontrollgruppe}

Von 27 weiblichen und 20 männlichen Probanden der Kontrollgruppe sind 13 Frauen und 9 Männer in einer der drei Proben positiv auf Candida getestet worden, wobei die Anzahl der Frauen mit nachgewiesener Candidabesiedlung, bezogen auf alle weiblichen Teilnehmer der Kontrollgruppe, mit 59\% einen größeren prozentualen Anteil zeigt als die Gruppe der männlichen Probanden mit 41\% (Tabelle 19). 


\begin{tabular}{|l|llllll|}
\hline Geschlecht & \multicolumn{2}{|l}{ Männlich } & \multicolumn{3}{l}{ Weiblich } & \multicolumn{3}{c|}{ Summe } \\
Anzahl & $\mathrm{n}$ & $(\%)$ & $\mathrm{n}$ & $(\%)$ & $\mathrm{n}$ & $(\%)$ \\
\hline Probanden insgesamt & 20 & $(42,55)$ & 27 & $(57,45)$ & 47 & $(100)$ \\
Probanden mit Candida & 9 & $(19,15)$ & 13 & $(27,66)$ & 22 & $(46,81)$ \\
Probanden mit Pilzbefall $(\mathrm{n}=22)$ & 9 & $(40,91)$ & 13 & $(59,09)$ & 22 & $(100)$ \\
männliche/ weibliche Probanden & 9 & $(45,00)$ & 13 & $(48,15)$ & 22 & $(46,81)$ \\
\hline
\end{tabular}

Tabelle 19: Geschlechterspezifische Candidabesiedlung (Kontrollgruppe)

\subsubsection{Mykologische Auswertung}

Von allen Isolaten der Pflegeheime wie auch der Kontrollgruppe war C. albicans mit $39 \%$ die am häufigsten isolierte Spezies, gefolgt von C. glabrata (36\%) und 8 weiteren Spezies (25\%). (Abbildung 6)

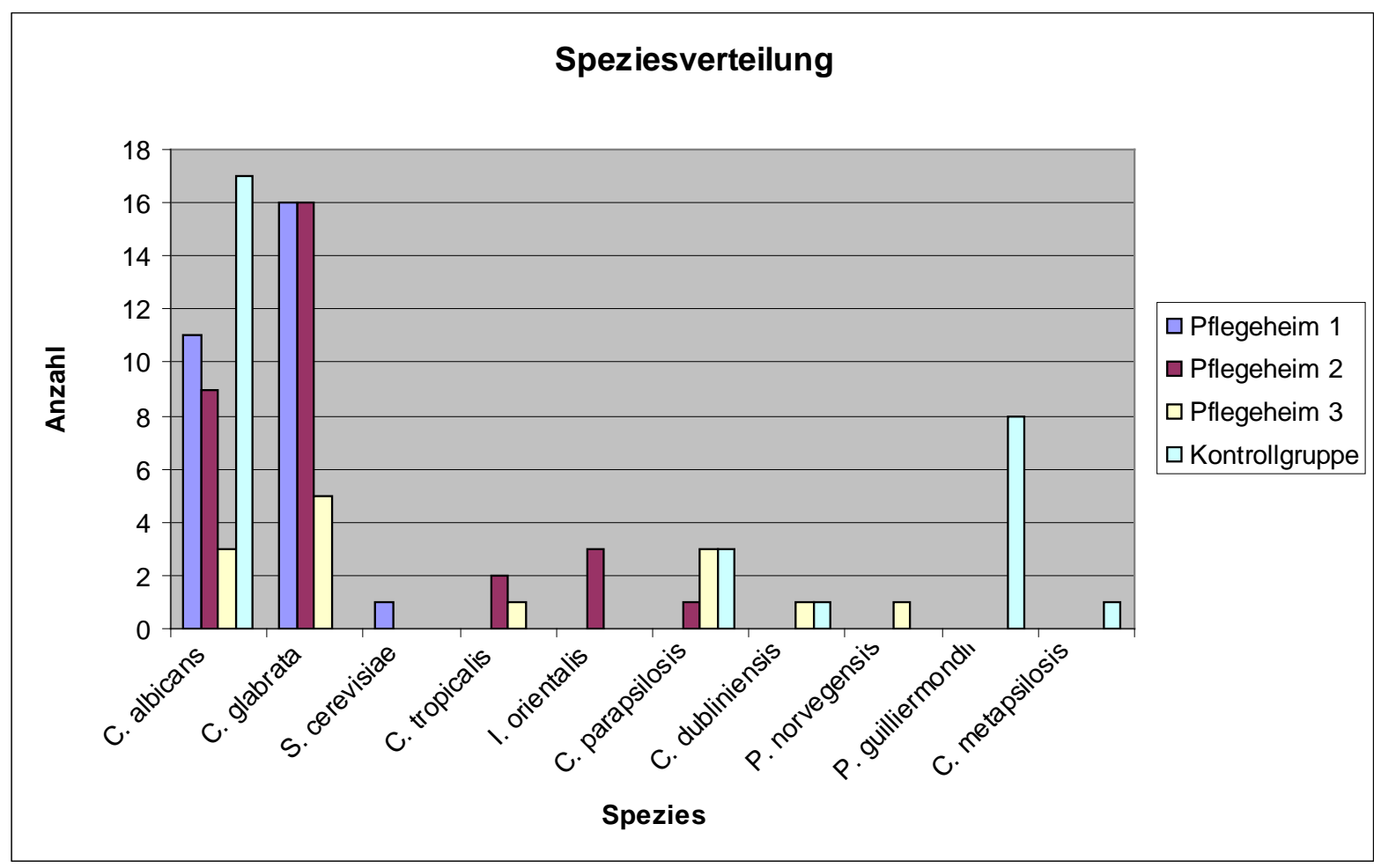

Abbildung 6: Speziesverteilung aller Gruppen

In 11 Proben wurden mehr als eine Spezies isoliert, so dass die Gesamtzahl aller Isolate aus den Pflegeheimen 73 beträgt (siehe oben). Dabei wurden in Pflegeheim 2 sechsmal 2 Spezies pro Proband und einmal 3 Spezies pro Proband gefunden. In Pflegeheim 3 konnten bei 4 Personen jeweils 2 Spezies aus einem Abstrich extrahiert werden.

Die Gesamtanzahl aller Isolate (inklusive der Isolate der Kontrollgruppe) beträgt 103. 


\section{Pflegeheim 1}

In Pflegeheim 1 (mit Schwerpunkt der Pflege demenzkranker Personen) konnte elfmal C. albicans (39\% der Isolate), sechzehnmal C. glabrata (57\%) und einmal S. cerevisiae (4\%) isoliert werden. Alle Isolate stammen ausschließlich aus den oralen Abstrichen.

\section{Pflegeheim 2}

In Pflegeheim 2 wurden insgesamt 31 Spezies isoliert. C. glabrata war mit 16 Isolaten (52\% aller Isolate dieser Gruppe) am häufigsten vertreten, gefolgt von $C$. albicans mit 29\%. 87\% der Isolate wurden der oralen Probe entnommen. C. glabrata wurde fünfzehnmal aus den oralen Abstrichen isoliert (56\% der oralen Hefen), C. albicans achtmal (30\%) und andere Spezies viermal (15\%). Aus den Proben der Leistenfalte und Zehenzwischenräume konnten jeweils 2 Spezies isoliert werden. In der Leistenfalte wurden C. glabrata und C. tropicalis isoliert, wohin gegen in den Proben des Zehenzwischenraumes jeweils einmal C. albicans und C. parapsilosis gefunden wurden.

\section{Pflegeheim 3}

10 der 14 Isolate aus Pflegeheim 3 wurden aus den oralen Abstrichen entnommen, wobei C. glabrata mit einer Anzahl von 4 Isolaten am häufigsten vorzufinden war. C. albicans wurde dreimal extrahiert sowie drei weitere Spezies. Insgesamt entstammten $71 \%$ der Isolate den oralen Abstrichen. In den Proben des Zehenzwischenraumes konnte dreimal C. parapsilosis und einmal C. glabrata nachgewiesen werden.

\section{Kontrollgruppe}

Innerhalb der Kontrollgruppe konnten insgesamt 30 positive Nachweise isoliert werden (in 6 Fällen wurden 2 und in einem Fall 3 Spezies gefunden). C. albicans wurde mit 57\% (17 von 30 Isolaten) am häufigsten angetroffen.

Ebenso wie P. guilliermondii mit 27\% (8 Isolate) ist C. parapsilosis mit 10\% (3 Isolate) sowie C. metapsilosis (1 Isolat, 3\%) häufiger in der Kontrollgruppe vertreten als in der Untersuchungsgruppe. C. dubliniensis wurde einmal nachgewiesen. 


\section{Häufigste Isolate}

Insgesamt wurden deutlich mehr Non-albicans-Hefen isoliert (Tabelle 20) als C. albicans.

Die Spezies, welche am häufigsten aus den Proben der Pflegeheime isoliert wurde, ist C. glabrata mit insgesamt 51\% (37 Isolate) aller Isolate der Pflegeheime (73 Isolate), gefolgt von Candida albicans mit 32\% (23 Isolate) und anderen Spezies mit $18 \%$ (13 Isolate).

\begin{tabular}{|l|l|l|l|l|l|l|}
\hline Non-albicans & Pflegeheim 1 & Pflegeheim 2 & Pflegeheim 3 & Summe Pflegeheime & Kontrollgruppe & Summe \\
\hline Anzahl $\mathrm{n}(\%)$ & $17(27,00)$ & $22(35,00)$ & $11(18,00)$ & $50(79,00)$ & $13(21,00)$ & $63(100)$ \\
\hline
\end{tabular}

Tabelle 20: Anzahl der Non-albicans-Isolate Vergleich Pflegeheime vs. Kontrollgruppe

\section{Seltene Isolate}

Saccharomyces cerevisiae, Candida tropicalis, Issatchenkia orientalis (Candida krusei) wurden ausschließlich innerhalb der Untersuchungsgruppe gefunden, wohingegen Pichia guilliermondii und Candida metapsilosis in der Untersuchungsgruppe nicht nachgewiesen werden konnten (Tabelle 21).

\begin{tabular}{|l|lllll|}
\hline Spezies/Gruppe & $\begin{array}{l}\text { Pflegeheim } 1 \\
\mathrm{n} \text { (oral/Leiste/Fuß) }\end{array}$ & $\begin{array}{l}\text { Pflegeheim } 2 \\
\mathrm{n} \text { (oral/Leiste/Fuß) }\end{array}$ & $\begin{array}{l}\text { Pflegeheim } 3 \\
\mathrm{n}(\text { oral/Leiste/Fuß) }\end{array}$ & $\begin{array}{l}\text { Kontrollgruppe } \\
\mathrm{n}(\text { oral/Leiste/Fuß) }\end{array}$ & $\begin{array}{l}\text { Summe } \\
(\%)\end{array}$ \\
\hline C. albicans & $11(11 / 0 / 0)$ & $9(8 / 0 / 1)$ & $3(3 / 0 / 0)$ & $17(13 / 2 / 2)$ & $40(38,83)$ \\
C. glabrata & $16(16 / 0 / 0)$ & $16(15 / 1 / 0)$ & $5(4 / 0 / 1)$ & $0(0 / 0 / 0)$ & $37(35,92)$ \\
C. parapsilosis & $0(0 / 0 / 0)$ & $1(0 / 0 / 1)$ & $3(0 / 0 / 3)$ & $3(0 / 1 / 2)$ & $7(6,80)$ \\
P. guilliermondii & $0(0 / 0 / 0)$ & $0(0 / 0 / 0)$ & $0(0 / 0 / 0)$ & $8(1 / 2 / 5)$ & $8(7,77)$ \\
C. tropicalis & $0(0 / 0 / 0)$ & $2(1 / 1 / 0)$ & $1(1 / 0 / 0)$ & $0(0 / 0 / 0)$ & $3(2,91)$ \\
I. orientalis & $0(0 / 0 / 0)$ & $3(3 / 0 / 0)$ & $0(0 / 0 / 0)$ & $0(0 / 0 / 0)$ & $3(2,91)$ \\
C. dubliniensis & $0(0 / 0 / 0)$ & $0(0 / 0 / 0)$ & $1(1 / 0 / 0)$ & $1(1 / 0 / 0)$ & $2(1,94)$ \\
S. cerevisiae & $1(1 / 0 / 0)$ & $0(0 / 0 / 0)$ & $0(0 / 0 / 0)$ & $0(0 / 0 / 0)$ & $1(0,97)$ \\
P. norvegensis & $0(0 / 0 / 0)$ & $0(0 / 0 / 0)$ & $1(1 / 0 / 0)$ & $0(0 / 0 / 0)$ & $1(0,97)$ \\
C. metapsilosis & $0(0 / 0 / 0)$ & $0(0 / 0 / 0)$ & $0(0 / 0 / 0)$ & $1(0 / 0 / 1)$ & $1(0,97)$ \\
Summe: $\mathrm{n}$ (\%) & $28(27,18)$ & $31(30,10)$ & $14(13,59)$ & $30(29,13)$ & $103(100)$ \\
\hline
\end{tabular}

Tabelle 21: Speziesverteilung der Gruppen (incl. Abstrichort)

\section{Vergleich: Verteilung der Spezies in Abhängigkeit von ihrem Abstrichort}

In den oralen Isolaten der Untersuchungsgruppe wurden 41 Non-albicans-Isolate gefunden, wobei Candida glabrata mit 35 am häufigsten vertreten war.

Dreimal wurde I. orientalis, zweimal C. tropicalis und jeweils einmal S. cerevisiae, C. dubliniensis und $P$. novergensis isoliert. 
In den Isolaten der Leistenfalte der Untersuchungsgruppe wurden nur zwei Nonalbicans-Spezies (C. glabrata und C. tropicalis) isoliert.

In der Zehenzwischenraumregion wurde einmal C. albicans und 4 Non-albicansSpezies nachgewiesen. Hierbei handelt es sich in 3 Fällen um C. parapsilosis und einmal um C. glabrata.

\section{Verteilung der Spezies in der Kontrollgruppe}

Innerhalb der Kontrollgruppe ist C. albicans im Vergleich zu C. glabrata häufiger vertreten. In den oralen Isolaten wurde dreizehnmal C. albicans isoliert, Nonalbicans-Spezies hingegen nur zweimal (C. dubliniensis und P. guilliermondii). Die Untersuchung der Isolate der Leistenfalte bei den Probanden der Kontrollgruppe ergab eine Verteilung zugunsten der Non-albicans-Isolate (zweimal P. guilliermondii und einmal C. parapsilosis). Zwei C. albicans-Spezies wurden ebenfalls isoliert.

Aus den Proben des dritten Abstrichortes wurden ebenfalls mehr Non-albicansSpezies (7) isoliert als C. albicans (2). Hierbei handelt es sich zum größten Teil um P. guilliermondii (5). C. parapsilosis wurde zweimal und C. metapsilosis einmal isoliert (Abbildung: 7).

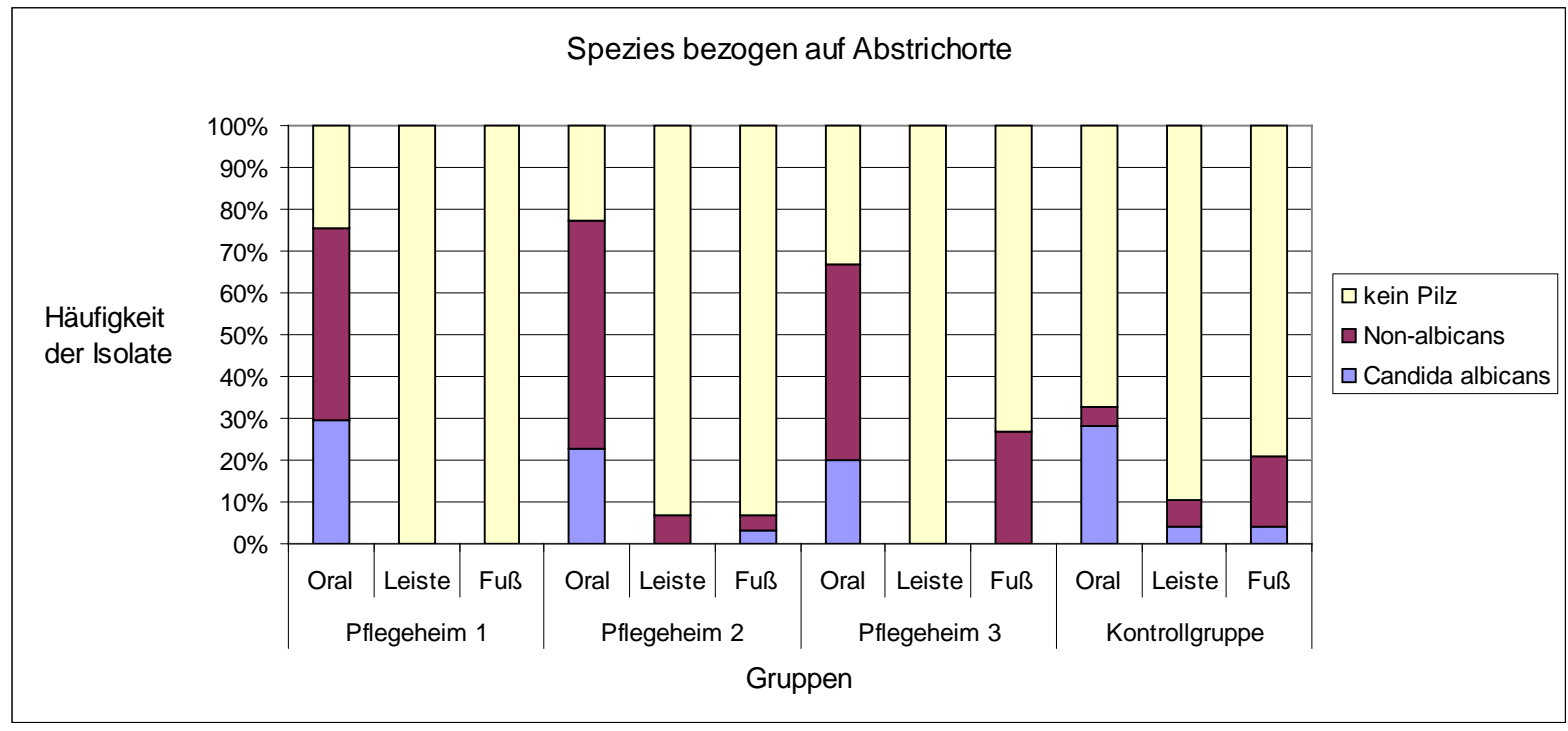

Abbildung 7: Häufigkeit der Isolate bezogen auf die Abstrichorte 


\section{Verteilung der Spezies auf die Altersgruppen}

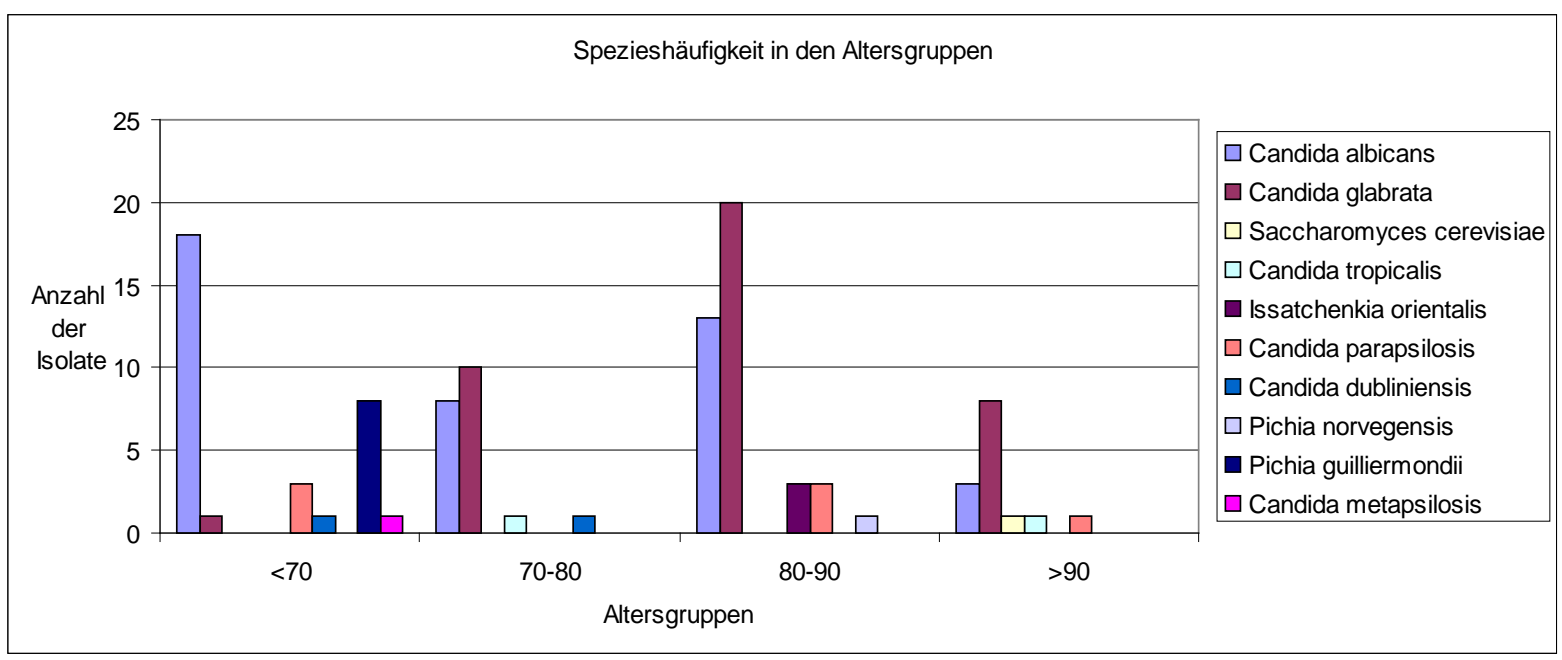

Abbildung 8: Verteilung der Spezies auf die Altersgruppen

Bei der Betrachtung der Absolutzahlen der Isolate verteilt auf die Altersgruppen zeigt sich ein Maximum an gefundenen Isolaten in der Gruppe der 80-90-Jährigen, gefolgt von der Gruppe der <70-Jährigen. Die geringste Anzahl an Isolaten wurde in der Altersgruppe $>90$ gefunden (Abbildung 8 ).

Vergleicht man die Anzahl gefundener Isolate mit der Anzahl der Probanden, so zeigt sich, dass sich auf jeden Probanden der älteren Altersgruppen (>70 Jahre) mehr Isolate verteilen, als auf die jüngere Altersgruppe. Hierbei kommen 40 Isolate auf 36 Probanden womit die Gruppe der 80-90-jährigen deutlich überwiegt.

In jeder Gruppe der >70-jährigen entfallen deutlich mehr Non-albicans- als C. albicans-Isolate auf einen Probanden. Umgekehrt verhält es sich mit den Isolaten der jüngeren Probandengruppe (<70 Jahre). Die größte Anzahl der dort gefundenen Isolate zählt in die Gruppe der C. albicans-Spezies (Tabelle 22).

\begin{tabular}{|c|c|c|c|c|c|}
\hline \multirow{2}{*}{$\begin{array}{l}\text { Altersgruppe } \\
\text { Anzahl }\end{array}$} & $<70$ & $71-80$ & $81-90$ & $>90$ & \multirow[b]{2}{*}{ Summe } \\
\hline & $(\%)$ & $(\%)$ & $(\%)$ & $(\%)$ & \\
\hline Probandenanzahl & 52 & 20 & 36 & 19 & 127 \\
\hline Candida insgesamt & $32(61,54)$ & $17(85,00)$ & $40(111,11)$ & $14(73,68)$ & 103 \\
\hline C.albicans & $18(34,62)$ & $6(30,00)$ & $13(36,11)$ & $3(15,79)$ & 40 \\
\hline Non-albicans & $14(26,92)$ & $11(55,00)$ & $27(75,00)$ & $11(57,98)$ & 63 \\
\hline
\end{tabular}

Tabelle 22: Verteilung der Probanden- und Isolate-Zahl auf die Altersgruppen 
In der Gruppe der Non-albicans-Isolate hebt sich besonders C. glabrata hervor (Abbildung 8). Die Spezies der Non-albicans-Gruppe welche in dieser jüngeren Altersgruppe nach $C$. albicans am zweithäufigsten vertreten ist, ist $P$. guilliermondii.

Betrachtet man die Verteilung der Spezies, die sich anhand der mikrobiologischen Untersuchung ergeben haben, so wird die Gruppe der Non-albicans-Spezies größtenteils von C. glabrata repräsentiert.

Insgesamt wurden dreiundsechzigmal Non-albicans-Spezies isoliert. Davon wurden siebenunddreißgmal C. glabrata und sechsundzwanzigmal weitere Non-albicansSpezies isoliert.

Diese 26 Isolate verteilen sich auf 8 verschiedene Spezies.

Gegenüberstellung von C. albicans- versus Non-albicans-Isolaten, in Abhängigkeit vom Alter

Trotz der zusätzlich (zu der Verteilung der C. albicans- und Non-albicans-Gruppe) aufgeführten Isolate ohne einen Pilznachweis lässt sich, mit zunehmendem Alter, ein sichtbarer Trend der C. albicans- sowie Non-albicans-Gruppe erkennen (Abbildung 9).

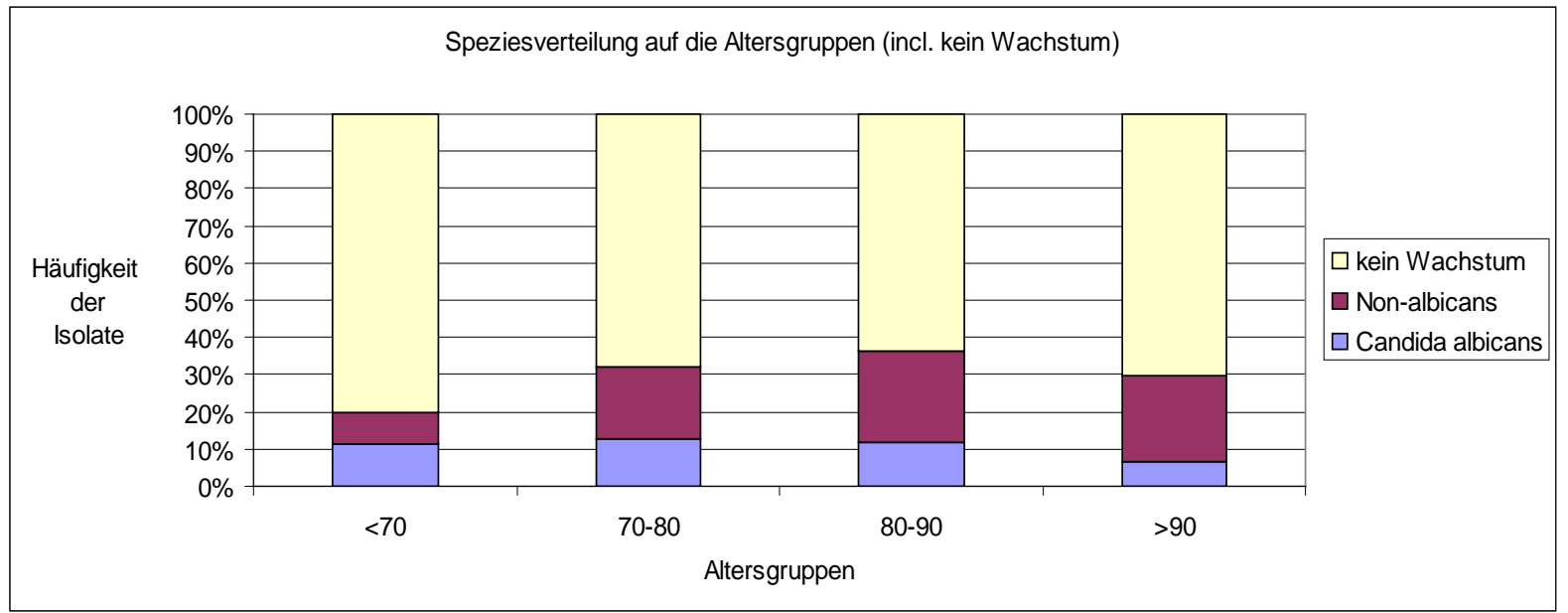

Abbildung 9: Speziesverteilung verteilt auf die Altersgruppen (incl. „kein Wachstum“)

Das Verteilungsmuster von C. albicans zu Non-albicans zeigt sich deutlicher ohne die Isolate ohne Wachstum (Abbildung 10). 


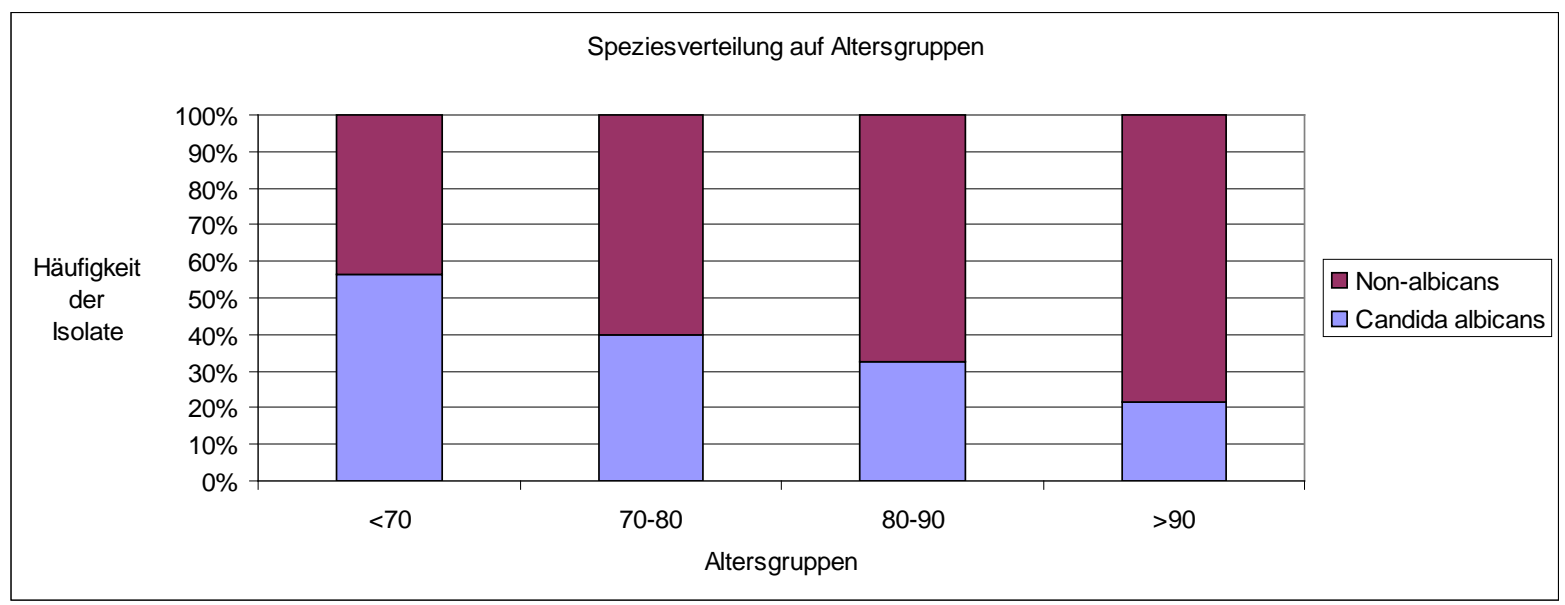

Abbildung 10: Speziesverteilung bezogen auf die Altersgruppen

Während in der jüngsten Probandengruppe der prozentuale Anteil der C. albicansSpezies mit 56\% am stärksten vertreten ist, nimmt dieser stetig in den aufsteigenden Altersgruppen (mit 40\% in der zweiten, 33\% in der dritten und $21 \%$ in der vierten Altersgruppe) ab.

Insgesamt wurden 39\% C. albicans- und 61\% Non-albicans-Spezies isoliert.

\subsubsection{Daten der Suszeptibilitätsprüfung}

Getestet wurden alle Isolate gegenüber den Polyen-Antimykotika Amphothericin B und Nystatin (MIC 50; getestet gemäß EUCAST eDef 7.1 in AM-3 Medium mit 0,4\% Glukose; abgelesen nach 36 Stunden). Des Weiteren wurden alle Isolate gegenüber den Azol-Antimykotika Fluconazol und Voriconazol getestet (MIC 50; getestet gemäß EUCAST eDef 7.1 in RPMI-Medium, individualisiert mit 0,4\% Glukose; abgelesen nach 17 Stunden). Zusätzlich wurden die Isolate gegenüber dem Echinocandin Micafungin untersucht (MIC 50; getestet gemäß EUCAST eDef 7.1 in RPMI Medium, individualisiert mit 0,4\% Glukose; abgelesen nach 17 Stunden).

Ein Isolat aus der Gruppe Pflegeheim 1, welches in der Speziesdifferenzierung als S. cerevisiae identifiziert wurde, lieferte keine Ergebnisse gegenüber Fluconazol, Voriconazol und Micafungin, da die MHK unter dem Messbereich lag. 
In gleicher Weise konnte ein C. albicans-Isolat aus der Gruppe Pflegeheim 2 nicht auf die drei o.g. Antimykotika getestet werden, da auch hier bei allen drei Testungen, wiederholt kein Wachstum innerhalb des Messbereichs verzeichnet werden konnte.

In der Gruppe der Polyene verteilt sich die größte Gruppe der Isolate (C. albicans wie auch Non-albicans) auf eine Hemmkonzentration um 0,125 $\mu \mathrm{g} / \mathrm{ml}$ Amphothericin B (Abbildung 11).

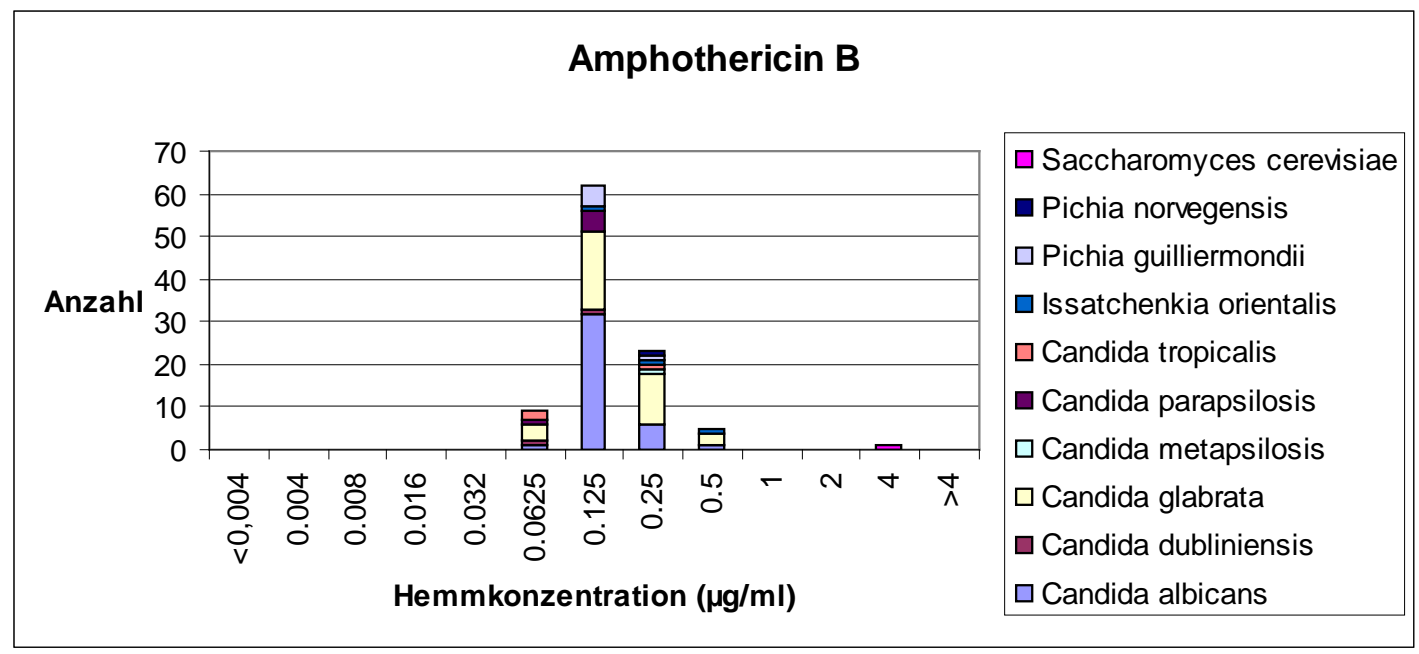

Abbildung 11: Daten der Resistenztestung: Amphothericin B

Gegenüber Nystatin zeigt sich ein ähnliches Verteilungsmuster um höhere Hemmkonzentrationen von 1 und $2 \mu \mathrm{g} / \mathrm{ml}$ (Abbildung 12).

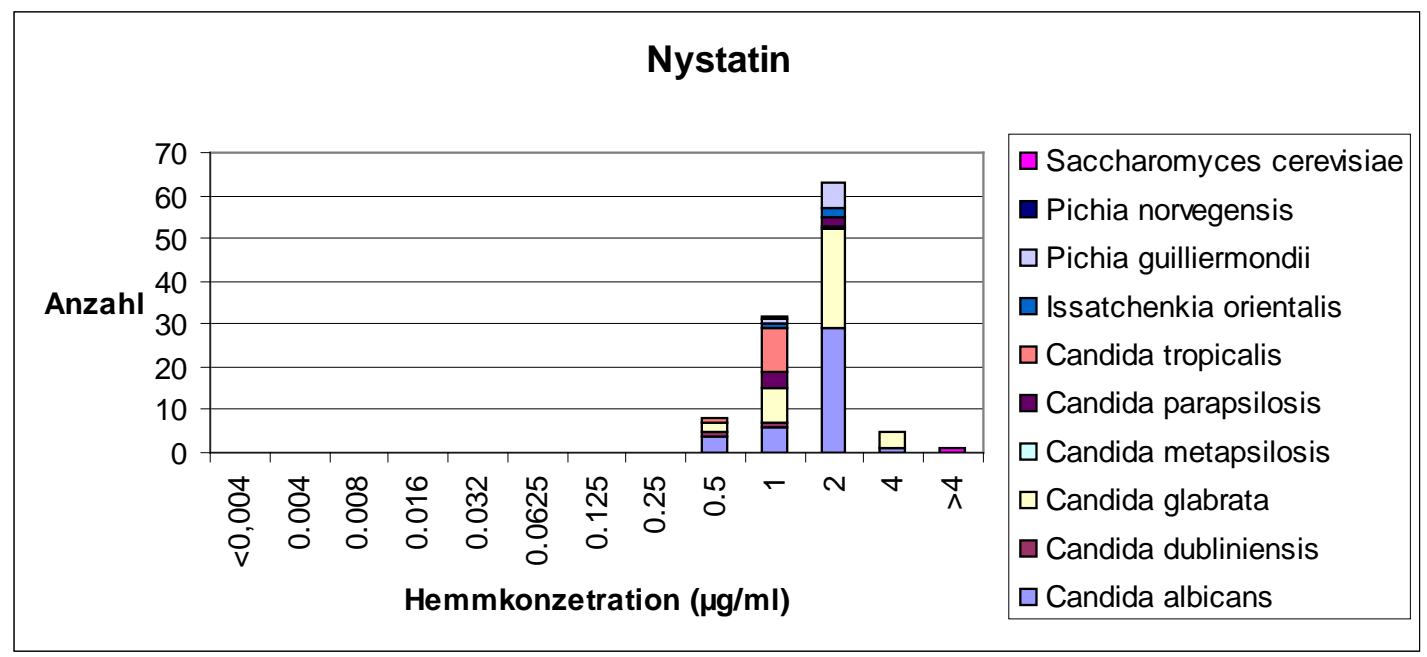

Abbildung 12: Daten der Resistenztestung: Nystatin 
C. albicans-Isolate sind besonders häufig bei einer Hemmkonzentration von <0,25 $\mu \mathrm{g} / \mathrm{ml}$ Fluconazol vertreten.

Bei den Isolaten mit einer Hemmkonzentration von $>8 \mu \mathrm{g} / \mathrm{ml}$ handelt es sich vornehmlich um Non-albicans-Spezies, größtenteils um C. glabrata (Abbildung 13).

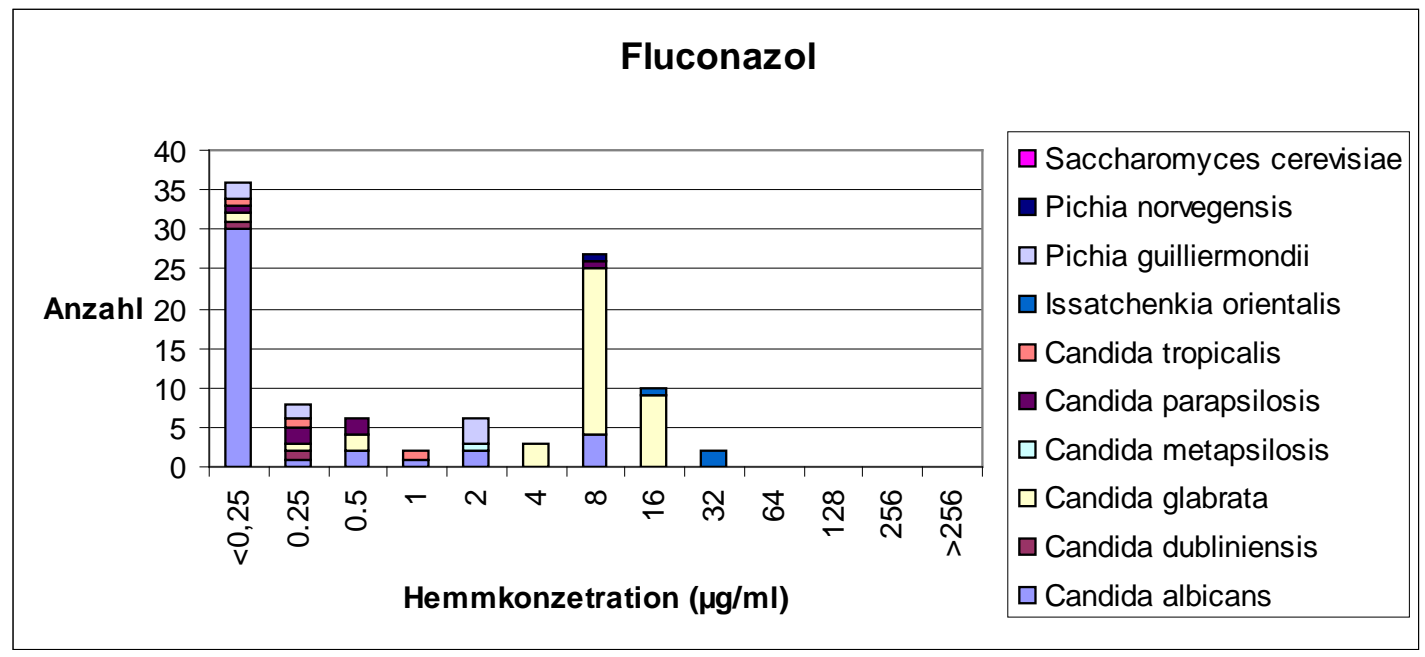

Abbildung 13: Daten der Resistenztestung: Fluconazol

Ebenso wie bei Fluconazol zeigt sich bei der Resistenztestung gegenüber Voriconazol eine deutliche Verschiebung der Gruppe der Non-albicans-Spezies in Richtung höherer Hemmkonzentrationen $(>0,125 \mu \mathrm{g} / \mathrm{ml})$, während C. albicans-Isolate größtenteils auf Hemmkonzentrationen $<0,032 \mu \mathrm{g} / \mathrm{ml}$ angesiedelt sind. (Abbildung 14).



Abbildung 14: Daten der Resistenztestung: Voriconazol

C. albicans- sowie C. glabrata-Isolate zeigen bei der Resistenztestung gegenüber Micafungin ein ähnliches Verteilungsmuster auf. 
Bei einer Hemmkonzentration von 2 und $4 \mu \mathrm{g} / \mathrm{ml}$ Antimykotikum wurden fast ausschließlich C. parapsilosis und C. metapsilosis gefunden (Abbildung 15).

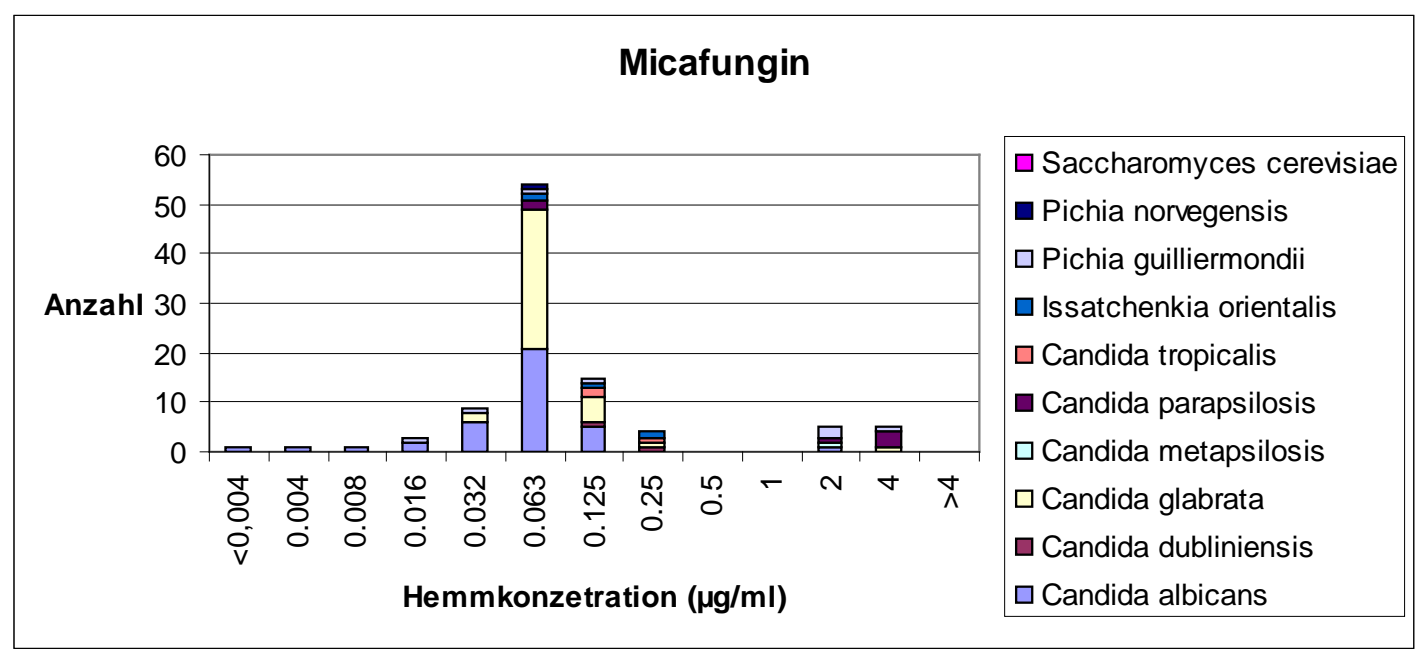

Abbildung 15: Daten der Resistenztestung: Micafungin

\subsection{Korrelation von mykologischen Befunden und klinischen Symptomen}

\subsubsection{Statistische Auswertung}

Zur besseren Übersicht werden ausschließlich die Ergebnisse, die eine statistische Signifikanz zeigten, aufgeführt.

Es wurden die mikrobiologischen Befunde (C. albicans/ Non-albicans/ kein klinisch auffälliger Befund bzw. keine Anzeichen einer Mykose) den Variablen aus den Befunderhebungsbögen gegenübergestellt.

Variablen sind u. a. das Alter, das Geschlecht, die Gruppeneinteilung, die eigenständige Mundhygiene, das Vorhandensein einer Prothese sowie die Prothesenhygiene, die Häufigkeit der Prothesenhygienemaßnahmen und der klinische Befund der Mundhöhle.

Die Gegenüberstellung der mikrobiologischen Ergebnisse mit den einzelnen Variablen (C. albicans/ Non-albicans/ kein mikrobiologischer Befund) wurde zu jedem der einzelnen Abstrichorte (Mundhöhle, Leistenfalte, Zehenzwischenraum), sowie 
der gesamten Anzahl der Isolate (unabhängig vom Abstrichort; "gesamt") untersucht (zur Übersicht der Gliederung der Datenanalyse siehe Anhang Abschnitt 9.5.).

Eine einzelne (univariate) Gegenüberstellung von mikrobiologischen Befunden und den Ergebnissen der Patientenbefragungsbögen zeigte mehrere statistisch signifikante Korrelationen.

\section{Untersuchungsgruppe (ohne Kontrollgruppe)}

Die statistische Auswertung der Untersuchungsgruppe (Pflegeheime 1-3 ohne die Kontrollgruppe) zeigten folgende signifikante Zusammenhänge:

Das Fehlen eines klinisch auffälligen mykologischen Befundes (negativer Befund) ungeachtet des Abstrichortes („gesamt“) korreliert signifikant mit der Pflegestufe ( $p=$ 0,04587). Je höher die Pflegestufe, desto höher war die Anzahl positiver mikrobiologischer Befunde.

Die Auswertung oraler mykologischer Befunde zeigte einen Zusammenhang zwischen „C. albicans“ und „Befund in der Mundhöhle“ (unabhängig von der Art und Ausprägung der Befunde) $(p=0,0154)$.

Die Befunde der Leistenfalte konnten in keinen signifikanten Zusammenhang mit den Ergebnissen des Befunderhebungsbogens gebracht werden.

Die Korrelation der mikrobiologischen Befunde des Zehenzwischenraums mit den Ergebnissen der Befunderhebungsbögen ergab einen signifikanten Zusammenhang von „Non-albicans-Spezies“ und „Auffälligkeiten im Abstrichareal“ ( $<<0,005)$ (ungeachtet dessen, um welche Art von Ausprägung es sich handelte) sowie „Nonalbicans-Spezies“ und „sonstige Auffälligkeiten: schuppige Hautveränderungen“ ( $p=$ $0,0375)$.

\section{Untersuchungsgruppe mit Kontrollgruppe}

In den Befunderhebungsbögen der Kontrollgruppe wurden Daten zu Alter, Geschlecht, eigenständiger Mundhygiene, Häufigkeit der Mundhygiene, Befund der Mundhöhle sowie dem Hautstatus erhoben. Somit konnten statistisch signifikante Korrelationen der Untersuchungs- und Kontrollgruppe für diese Variablen berechnet werden. 


\section{Gesamtheit der Isolate}

Die Verteilung von jedweden C. albicans-Isolaten innerhalb der Untersuchungs- und Kontrollgruppe, unabhängig vom Abstrichort („gesamt“), ergab einen signifikanten Zusammenhang mit auffälligen klinischen Befunden der Mundhöhle (unabhängig von der Art des Befundes dieser Region; $p=0,0091$ ) (Tabelle 23).

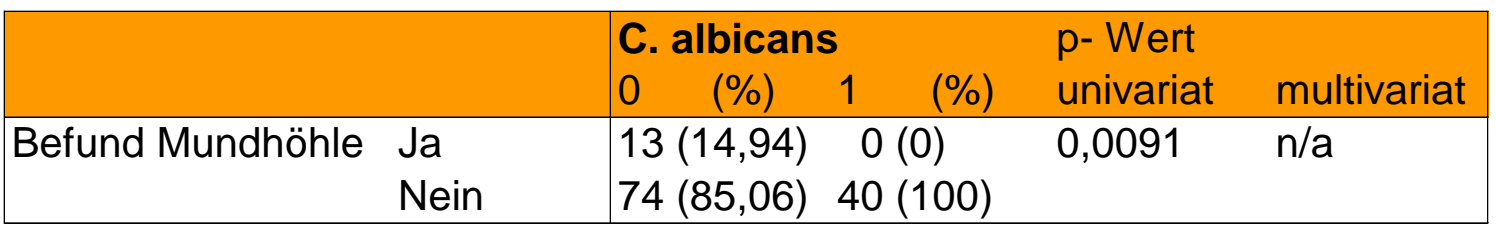

Tabelle 23: Gesamtheit der Isolate: $C$. albicans

Die Verteilung der Non-albicans-Spezies zeigte einen deutlichen Zusammenhang zu dem Alter der Probanden aller Gruppen ( $p=0,000014)$. Mit dem Alter der Probanden nahm auch die Häufigkeit von Non-albicans-Spezies zu.

Ebenfalls zeigte die Gruppe der Non-albicans-Spezies einen Zusammenhang zu der Gruppeneinteilung (Pflegeheim 1-3, Kontrollgruppe) $(p=0,00038)$. Non-albicansSpezies wurden deutlich häufiger in der Untersuchungsgruppe nachgewiesen als in der Kontrollgruppe. Besonders deutlich zeigte sich eine Diskrepanz zwischen den Pflegeheimen 1 (mit Schwerpunkt Demenzpflege) und 2 und der Kontrollgruppe (Tabelle 24).

Die „eigenständige Mundhygiene“ ( $p=0,04681)$ konnte ebenfalls mit der Verteilung der Non-albicans-Isolate in Verbindung gebracht werden (Tabelle 24).

Das Tragen einer Prothese korrelierte mit der Gruppe der Non-albicans-Spezies ( $p=$ 0,0174). Bei Prothesenträgern konnten häufiger Non-albicans-Spezies nachgewiesen werden als Probanden ohne Zahnersatz.

Zusätzlich zeigten „sonstige Mundhygiene-Artikel (0-2)“ ( $p=0,0427)$, ein klinisch auffälliger „Befund der Mundhöhle“ ( $p=0,04131)$ und die Verteilung „Untersuchungsvs. Kontrollgruppe“ ( $p=0,00008)$ einen Zusammenhang mit den analysierten Nonalbicans-Spezies.

Hierbei zeigten sich statistisch signifikante Korrelationen zwischen den Probanden der Pflegeheime 2 und 3 und der Verteilung aller Non-albicans-Spezies auf die Abstrichorte (Pflegeheim 2: $p=0,03304$; Pflegeheim 3: $p=0,04131$ ). 
Bei beiden Einrichtungen war die Anzahl der Probanden mit positivem Befund höher als die Anzahl der Probanden mit negativem Befund (im Vergleich zum gesamten Probandenkollektiv).

In Pflegeheim 1 wurde keine Korrelation zu der Verteilung der Non-albicans-Isolate festgestellt.

Ein Trend zeigte sich bezüglich der Prothesenhygiene ab $(p=0,05126)$ (Tabelle 24).

\begin{tabular}{|c|c|c|c|c|c|}
\hline & \multicolumn{2}{|c|}{ Non-albicans } & \multicolumn{2}{|l|}{ p- Wert } \\
\hline & & $0 \quad(\%)$ & $(\%)$ & univariat & multivariat \\
\hline \multicolumn{2}{|l|}{ Alter } & & & 0,000014 & 0,048305 \\
\hline \multicolumn{2}{|l|}{ Eigenständige } & $16(21,92)$ & $21(39,62)$ & 0,04681 & 0,699214 \\
\hline \multirow{3}{*}{$\begin{array}{l}\text { Mundhygiene } \\
\text { Prothese }\end{array}$} & nein & $57(78,08)$ & $32(60,83)$ & & \\
\hline & ja & $24(32,43)$ & $29(54,72)$ & 0,0174 & 0,952817 \\
\hline & nein & $50(67,57)$ & $24(45,28)$ & & \\
\hline \multirow[t]{2}{*}{ Befund Mundhöhle } & ja & $44(83,02)$ & $9(16,98)$ & 0,04131 & 0,278393 \\
\hline & nein & $70(94,59)$ & $4 \quad(5,41)$ & & \\
\hline \multirow[t]{2}{*}{ Untersuchungsgruppe } & ja & $36(48,65)$ & $44(83,02)$ & 0,00008 & $\mathrm{n} / \mathrm{a}$ \\
\hline & nein & $38(51,35)$ & $9(16,98)$ & & \\
\hline \multirow[t]{2}{*}{ Pflegeheim 2} & ja & $12(16,22)$ & $18(33,96)$ & 0,03304 & $\mathrm{n} / \mathrm{a}$ \\
\hline & nein & $62(83,78)$ & $35(66,04)$ & & \\
\hline \multirow[t]{2}{*}{ Pflegeheim 3} & ja & $4 \quad(5,41)$ & $9 \quad(16,98)$ & 0,04131 & $\mathrm{n} / \mathrm{a}$ \\
\hline & nein & $70(94,59)$ & $44(83,02)$ & & \\
\hline \multirow[t]{3}{*}{ Sonst. MH-Artikel } & $0=$ keine $\mathrm{MH}$-Artikel & $96(93,24)$ & $43(81,13)$ & 0,0427 & 0,996067 \\
\hline & 1 = Mundspülung & $4 \quad(5,41)$ & $10(18,87)$ & & 0,995439 \\
\hline & $2=$ Adstringentien & $1 \quad(1,35)$ & $0 \quad(0,00)$ & & $\mathrm{n} / \mathrm{a}$ \\
\hline \multirow[t]{4}{*}{ Gruppen } & PFE 1 & $20(27,03)$ & $17(32,08)$ & 0,00038 & 0,143324 \\
\hline & PFE 2 & $12(16,22)$ & $18(33,96)$ & & 0,467751 \\
\hline & PFE 3 & $4 \quad(5,41)$ & $9 \quad(16,98)$ & & 0,984695 \\
\hline & Kontrl. & $38(51,35)$ & $9 \quad(16,98)$ & & $\mathrm{n} / \mathrm{a}$ \\
\hline \multirow[t]{4}{*}{ Prothesenhygiene } & $0 \mathrm{mal} / \mathrm{Tag}$ & $52(70,27)$ & $26(49,06)$ & 0,05126 & 0,848368 \\
\hline & $1 \mathrm{mal} / \mathrm{Tag}$ & $1 \quad(1,35)$ & $4(7,55)$ & & 0,417985 \\
\hline & $2 \mathrm{mal} / \mathrm{Tag}$ & $19(25,68)$ & $22(41,51)$ & & 0,721623 \\
\hline & $3 \mathrm{mal} / \mathrm{Tag}$ & $2 \quad(2,70)$ & $1(1,89)$ & & $\mathrm{n} / \mathrm{a}$ \\
\hline
\end{tabular}

Tabelle 24: Gesamtheit der Isolate: Non-albicans

Mit dem Fehlen eines mikrobiologischen Befundes (negativer Befund) konnten das (jüngere) Alter der Probanden $(p=0,001194)$ (Tabelle 25), die Gruppenverteilung (PFE 1-3, Kontrollgruppe;) $(p=0,02777)$ (Tabelle 25) und die Probandenverteilung auf Untersuchungs- vs. Kontrollgruppe $(p=0,0038)$ in Zusammenhang gebracht werden (Tabelle 25).

Es zeigte sich, dass jüngere Probanden (und somit Probanden der Kontrollgruppe) häufiger keinen mikrobiologischen Befund aufwiesen. 
Gleichermaßen korrelierte die „eigenständige Mundhygiene“ mit der Verteilung eines fehlenden mikrobiologischen Befundes $(p=0,02311)$. Die Anzahl positiver mikrobiologischer Befunde ist bei Personen, welche auf Hilfe bei der Mundhygiene angewiesen sind, höher. Probanden mit eigenständig durchgeführter Mundhygiene zeigten seltener Anzeichen von Hefebesiedlungen (Tabelle 25).

„Kein Befund“ mit der Ausprägung „1“ bedeutet in diesem Zusammenhang, dass keine Pilzbesiedlung in den Isolaten gefunden wurde. Die Ausprägung „0“ hingegen bedeutet hierbei jedoch, dass Hefen nachgewiesen wurden.

\begin{tabular}{|ll|llllll|}
\hline & & \multicolumn{3}{c}{ kein Befund } & p- Wert & \\
& & 0 & $(\%)$ & 1 & $(\%)$ & univariat & multivariat \\
\hline Alter & & & & 0,001194 & 0,228881 \\
Eigenständige & ja & $30(36,59)$ & 7 & $(15,91)$ & 0,02311 & 0,308524 \\
Mundhygiene & nein & $52(63,41)$ & $37(84,09)$ & & \\
Untersuchungsgruppe & ja & $60(72,29)$ & $20(45,45)$ & 0,0038 & n/a \\
& nein & $23(27,71)$ & $24(54,55)$ & & \\
Gruppen & PFE 1 & $28(33,37)$ & 9 & $(20,45)$ & 0,02777 & 0,619156 \\
& PFE 2 & $23(27,71)$ & 7 & $(15,91)$ & & 0,722554 \\
& PFE 3 & $9(10,84)$ & 4 & $(9,09)$ & & 0,745302 \\
& Kontrl. & $23(27,71)$ & $24(54,55)$ & & n/a \\
\hline
\end{tabular}

Tabelle 25: Gesamtheit der Isolate: kein klinisch auffälliger Befund

\section{$\underline{\text { Orale Isolate }}$}

Die Datenanalyse der oralen Isolate zu „C. albicans" zeigte eine signifikante Korrelation zu klinisch auffälligen Befunden der Mundhöhle ( $p=0,01899)$ (Tabelle 26).

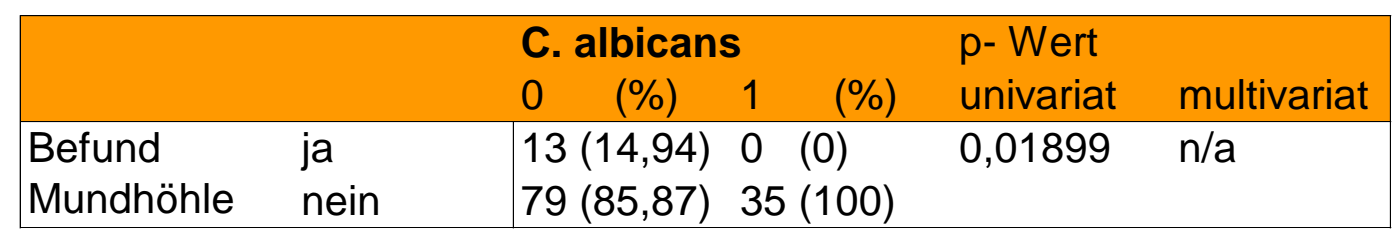

Tabelle 26: orale Isolate: $C$. albicans

Die Verteilung oraler Non-albicans-Isolate zeigte statistische Zusammenhänge zu dem Alter $(p<0,005)$, der Gruppeneinteilung $(p<0,005)$ der eigenständigen Mundhygiene $(p=0,00364)$, der Verwendung sonstiger Mundhygiene-Artikel $(p=$ 0,03155), den Probanden mit/ ohne herausnehmbaren Zahnersatz $(p=0,00109)$, der 
Prothesenhygiene $(p=0,01124)$, Befunden der Mundhöhle jeglicher Art $(p=0,03362)$ sowie der Einteilung in Untersuchungs- vs. Kontrollgruppe ( $p<0,005$; Tabelle 27).

Ebenso gab es eine signifikante Korrelation der Probanden des Pflegeheimes 2 mit der Verteilung oraler Non-albicans-Isolate.

Hierbei zeigten allein die Probanden des zweiten Pflegeheims eine statistische Signifikanz $(p=0.00388)$ in der Anzahl der positiven und negativen Pilzbefunde (Tabelle 27). Erneut war die Anzahl der Probanden mit positivem Pilzbefund deutlich höher. Auch in dem dritten Pflegeheim war die Anzahl der positiven Pilzbefunde des oralen Abstrichortes erhöht, zeigten jedoch keine statistische Signifikanz im Vergleich mit den anderen Pflegeheimen (Tabelle 27).

Ähnlich den Ergebnissen der Gesamtheit der Non-albicans-Isolate wurden mikrobiologische Befunde häufiger bei Probanden höheren Alters (Probanden der Untersuchungsgruppe), Probanden die auf zusätzliche Hilfe bei der Mundhygiene angewiesen waren und Probanden mit herausnehmbarem Zahnersatz festgestellt. (Tabelle 27)

\begin{tabular}{|c|c|c|c|c|c|}
\hline & \multicolumn{2}{|c|}{ Non-albicans } & \multicolumn{2}{|l|}{$p-$ Wert } \\
\hline & & $(\%)$ & (\%) & univariat & multivariat \\
\hline \multicolumn{2}{|l|}{ Alter } & & & $<0,005$ & 0,113765 \\
\hline \multicolumn{2}{|l|}{ Eigenständige } & $17(20,48)$ & $20(46,51)$ & 0,00364 & 0,832892 \\
\hline \multirow{2}{*}{$\begin{array}{l}\text { Mundhygiene } \\
\text { Prothese }\end{array}$} & nein & $66(79,52)$ & $23(53,49)$ & & \\
\hline & ja & $26(30,95)$ & $27(62,79)$ & 0,00109 & 0,734325 \\
\hline & nein & $58(69,05)$ & $16(37,21)$ & & \\
\hline Befund Mundhöhle & $\begin{array}{l}\text { ja } \\
\text { nein }\end{array}$ & $\begin{array}{ll}5 & (5,95) \\
79 & (94,05)\end{array}$ & $\begin{array}{ll}8 & (18,6) \\
35 & (81,40)\end{array}$ & 0,03362 & 0,382460 \\
\hline Untersuchungsgruppe & $\begin{array}{l}\text { ja } \\
\text { nein }\end{array}$ & $\begin{array}{l}39(46,43) \\
45(53,57)\end{array}$ & $\begin{array}{l}41(95,35) \\
2 \quad(4,65)\end{array}$ & $<0,005$ & $\mathrm{n} / \mathrm{a}$ \\
\hline \multirow[t]{2}{*}{ Pflegeheim 2} & ja & $13(15,48)$ & $17(39,53)$ & 0,00388 & $\mathrm{n} / \mathrm{a}$ \\
\hline & nein & $71(84,52)$ & $26(60,47)$ & & \\
\hline \multirow[t]{3}{*}{ Sonst. MH-Artikel } & $0=$ keine $\mathrm{MH}$-Artikel & $78(92,86)$ & $34(79,07)$ & 0,03155 & 0,995906 \\
\hline & 1 = Mundspülung & $5 \quad(5,95)$ & $9 \quad(20,93)$ & & 0,995480 \\
\hline & $2=$ Adstringentien & $1 \quad(1,19)$ & $0 \quad(0,00)$ & & $\mathrm{n} / \mathrm{a}$ \\
\hline \multirow[t]{4}{*}{ Gruppen } & PFE 1 & $20(23,81)$ & $17(39,53)$ & $<0,005$ & 0,966401 \\
\hline & PFE 2 & $13(15,48)$ & $17(39,53)$ & & 0,704964 \\
\hline & PFE 3 & $6 \quad(7,14)$ & $7 \quad(16,28)$ & & 0,879039 \\
\hline & Kontrl. & $45(53,57)$ & $2(4,65)$ & & $\mathrm{n} / \mathrm{a}$ \\
\hline \multirow[t]{4}{*}{ Prothesenhygiene } & $0 \mathrm{mal} / \mathrm{Tag}$ & $60(71,43)$ & $18(41,86)$ & 0,01124 & 0,621870 \\
\hline & $1 \mathrm{mal} / \mathrm{Tag}$ & $2(2,38)$ & $3(6,98)$ & & 0,744927 \\
\hline & $2 \mathrm{mal} / \mathrm{Tag}$ & $20(23,81)$ & $21(48,84)$ & & 0,723236 \\
\hline & $3 \mathrm{mal} / \mathrm{Tag}$ & $2(2,38)$ & $1(2,33)$ & & $\mathrm{n} / \mathrm{a}$ \\
\hline
\end{tabular}

Tabelle 27: Orale Isolate: Non-albicans 
Analysen zur Prothesenhygiene (Tabelle 27) zeigen, dass die tatsächliche Anzahl von Probanden mit einem positiven bzw. negativen Pilzbefund etwa gleich verteilt war, unabhängig davon, wie oft die Prothesenhygiene durchgeführt wurde. Lediglich in der Gruppe ohne Prothesenhygiene („,“) sind mehr Probanden ohne Pilzbefall ( $\mathrm{n}=$ 60) vorzufinden als mit positivem Pilznachweis $(n=18)$. Diese hohe Anzahl von Probanden ohne Prothesenhygiene kam durch die Probanden der Kontrollgruppe zustande, da in der Kontrollgruppe keine Prothesenhygiene durchgeführt wurde.

Bei alleiniger Betrachtung der Untersuchungsgruppe (exklusive der Kontrollgruppe) ließen sich keine statistisch signifikanten p-Werte zu den Variablen „Prothese“ und „sonstige Mundhygieneartikel“ berechnen (Tabelle 28).

\begin{tabular}{|c|c|c|}
\hline & \multicolumn{2}{|c|}{ p-Werte: } \\
\hline & Oral albicans & Oral Non-albicans \\
\hline „weitere MH-Artikel“ & 0.13806 & 0.34827 \\
\hline Prothesenhygiene & 0.13673 & 0.90995 \\
\hline
\end{tabular}

Tabelle 28: Prothese und sonstige MH-Artikel: Untersuchungsgruppe

Die Untersuchungsgruppe dieser Studie zeigt ohne Beteiligung der Kontrollgruppe keine signifikanten Zusammenhänge zu den o. g. Variablen (Tabelle 28).

Ein fehlender mikrobiologischer Befund der oralen Isolate zeigt ebenfalls signifikante Korrelationen mit einem jüngeren Alter $(p=0,00001)$, der Gruppeneinteilung (Kontrollgruppe) $(p=0,0003)$, der eigenständige Mundhygiene $(p=0,00297)$, dem Nicht-Tragen einer Prothese $(p=0,01099)$, der Einteilung in Untersuchungs- vs. Kontrollgruppe $(p=0,00003)$ sowie der Aufteilung der Probanden in Pflegeheim 1 und andere $(p=0,0168)$ (Tabelle 29).

Die Ausprägung „1“ steht hierbei erneut für das Fehlen eines mikrobiologischen Befundes, sowie die "0“ für das Vorhandensein (im Sinne von: „nein, es wurden Hefen nachgewiesen“). 


\begin{tabular}{|ll|llllll|}
\hline & \multicolumn{5}{c|}{ kein Befund } & \multicolumn{3}{c|}{ p- Wert } \\
& & 0 & $(\%)$ & 1 & $(\%)$ & univariat & multivariat \\
\hline Alter & & & & & 0,000010 & 0,459962 \\
Eigenständige & ja & $29(39,73)$ & 8 & $(15,09)$ & 0,00297 & 0,466412 \\
Mundhygiene & nein & $44(60,27)$ & $45(84,91)$ & & \\
Prothese & ja & $38(51,35)$ & $15(28,30)$ & 0,01099 & 0,738374 \\
& nein & $36(48,65)$ & $38(71,70)$ & & \\
Untersuchungsgruppe & ja & $58(78,38)$ & $22(41,51)$ & 0,00003 & n/a \\
& nein & $16(21,62)$ & $31(58,49)$ & & \\
Pflegeheim 1 & ja & $28(37,84)$ & $9(16,98)$ & 0,0168 & n/a \\
& nein & $46(62,16)$ & $44(83,02)$ & & \\
Gruppen & PFE 1 & $28(37,84)$ & 9 & $(16,98)$ & 0,0003 & 0,604019 \\
& PFE 2 & $22(29,73)$ & 8 & $(15,09)$ & & 0,732456 \\
& PFE 3 & $8(10,81)$ & 5 & $(9,43)$ & & 0,817992 \\
& Kontrl. & $16(21,62)$ & $31(58,49)$ & & n/a \\
\hline
\end{tabular}

Tabelle 29: Orale Isolate: kein klinisch auffälliger Befund

\section{Isolate der Leistenfalte}

An der Leistenfalte konnten keine signifikanten Zusammenhänge zwischen klinischen Befunden einerseits und dem mikrobiologischem Befund andererseits errechnet werden.

\section{Isolate der Zehenzwischenräume}

Ebenso wie die Berechnungen zu den Abstrichen der Leistenfalte zeigte die Verteilung der $C$. albicans-Isolate der Zehenzwischenräume keinen signifikanten Zusammenhang mit den klinischen Befunden.

Non-albicans-Isolate der Zehenzwischenräume konnten statistisch hingegen mit der Geschlechterverteilung ( $p=0,03054)$ in Verbindung gebracht werden.

Statistisch war die Anzahl männlicher Probanden mit positivem Non-albicans-Befund höher als die Anzahl weiblicher Studienteilnehmer.

Bezüglich der Proben der Zehenzwischenräume zeigte sich, dass Non-albicansSpezies signifikant häufiger in der Kontrollgruppe als in der Untersuchungsgruppe vorgefunden wurden $(p=0,03788)$ (Tabelle 30$)$.

Hierbei wiesen 7 der 47 Probanden der Kontrollgruppe Non-albicans-Spezies auf, was einen prozentualen Anteil von 70\% aller Probanden mit positivem Non-albicansBefall an diesem Abstrichort ausmacht (3 der übrigen 80 Probanden, welche nicht 
zur Kontrollgruppe zählen, hatten ebenfalls einen positiven Pilznachweis in dieser Abstrichregion) (Tabelle 30).

Außerdem konnten die Gruppenverteilung $(p=0,04171)$ sowie die Aufteilung in Pflegeheim 1 und andere, mit einem mikrobiologischen Befund in Verbindung gebracht werden (Tabelle 30).

Ein signifikanter p-Wert von 0.03393 ergab sich hierbei für die Probanden des Pflegeheims 1. In Pflegeheim 1 konnten keine Pilzsporen an diesem Abstrichort nachgewiesen werden.

Demzufolge zeigt sich auch ein signifikanter Zusammenhang bei dem Fehlen eines Pilznachweises (Tabelle 31). Alle positiven Befunde (an diesem Abstrichort) wurden ausschließlich in anderen Pflegeheimen gefunden.

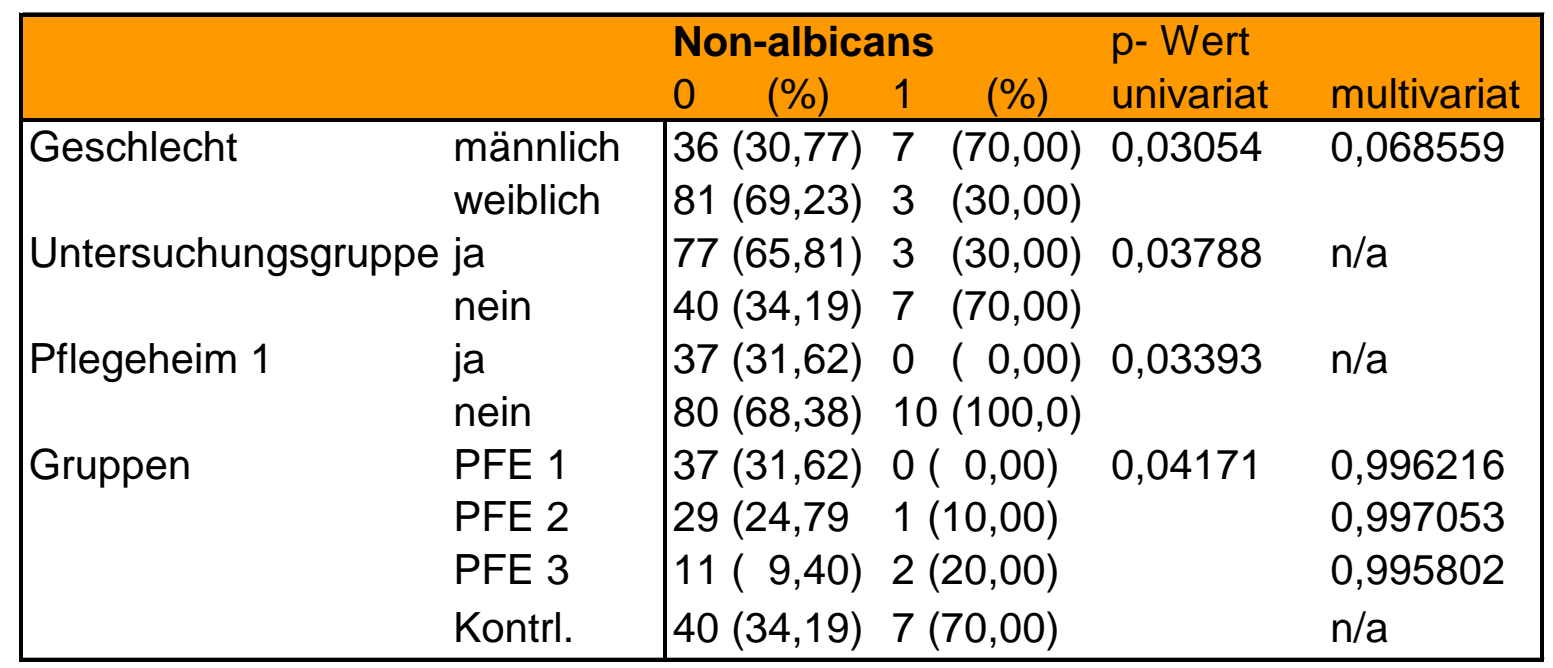

Tabelle 30: Isolate Zehenzwischenraum: Non-albicans

Um zu charakterisieren, welche Probanden keinen pathologischen Befund aufwiesen (hier: in den Zehenzwischenräumen), wurde das Merkmal „kein mikrobiologischer Befund im Zehenzwischenraum" mit klinischen und demografischen Merkmalen korreliert (Tabelle 31).

Weibliche Probanden wiesen seltener Pilze in den Abstrichen der Zehenzwischenräume auf als männliche Probanden $(p=0,01008)$.

Die Verteilung der Probanden auf die Untersuchungsgruppe korrelierte mit dem Fehlen von Pilzbefunden an diesem Abstrichort. Dies zeigt sich in der 
Gruppeneinteilung (PFE 1-3, Kontrollgruppe) ( $p=0,02833$ ), sowie der Einteilung in Untersuchungs- vs. Kontrollgruppe $(p=0,01554)$. Die Pilzbefunde an diesem Abstrichort waren in der Kontrollgruppe deutlich häufiger.

Im Pflegeheim 1 zu sein $(p=0,01055)$, sowie das höhere Alter der Probanden $(p=$ 0,035642 ) korrelierte ebenfalls mit dem fehlenden mikrobiologischen Befund an diesem Abstrichort (Tabellen 31).

\begin{tabular}{|c|c|c|c|c|c|}
\hline & \multicolumn{2}{|c|}{ kein Befund } & \multicolumn{2}{|l|}{$p$ - Wert } \\
\hline & & (\%) & (\%) & univariat & multivariat \\
\hline Alter & & & & 0,035642 & 0,124769 \\
\hline \multirow[t]{2}{*}{ Geschlecht } & männlich & $9 \quad(69,23)$ & $34(29,82)$ & 0,01008 & 0,058801 \\
\hline & weiblich & $4 \quad(30,77)$ & $80(70,18)$ & & \\
\hline \multirow[t]{2}{*}{ Untersuchungsgruppe } & ja & $4 \quad(30,77)$ & $76(66,67)$ & 0,01554 & $\mathrm{n} / \mathrm{a}$ \\
\hline & nein & $9 \quad(69,23)$ & $38(33,33)$ & & \\
\hline \multirow[t]{2}{*}{ Pflegeheim 1} & ja & $0 \quad(0,00)$ & $37(32,46)$ & 0,01055 & $\mathrm{n} / \mathrm{a}$ \\
\hline & nein & $13(100,0)$ & $77(67,54)$ & & \\
\hline \multirow[t]{4}{*}{ Gruppen } & PFE 1 & $0(0,00)$ & $37(32,46)$ & 0,02833 & 0,995668 \\
\hline & PFE 2 & $2(15,38)$ & $28(24,56)$ & & 0,997932 \\
\hline & PFE 3 & $2(15,38)$ & $11(9,65)$ & & 0,997123 \\
\hline & Kontrl. & $9(69,23)$ & $38(33,33)$ & & $\mathrm{n} / \mathrm{a}$ \\
\hline
\end{tabular}

Tabelle 31: Isolate Zehenzwischenraum: kein klinisch auffälliger Befund

\subsubsection{Logistische Regression}

Mit Hilfe eines multivariaten Modells wurde untersucht, ob der Zusammenhang mehrerer, im Einzelnen abhängiger Variablen (mit signifikanten p-Werten) bzw. deren gegenseitiger Einfluss mit den mikrobiologischen Befunden in Verbindung gebracht werden können.

Wie bereits oben erwähnt, konnten aufgrund der geringen Variablenanzahl bzw. des Fehlens von Ergebnissen der univariaten Berechnungen keine weiteren Rechenmodelle der logistischen Regression zu dem mikrobiologischen Befund „C. albicans“, der gesamten Anzahl der Isolate, der Isolate der Mundhöhle sowie des Zehenzwischenraumes erstellt werden. 
Ebenso konnten keine multivarianten Berechnungen zu allen drei Variablen ( $C$. albicans/ Non-albicans/ kein klinisch auffälliger Befund bzw. negativer Befund) bezüglich der mikrobiologischen Befunde der Leistefalte aufgrund fehlender univariater Ergebnisse gemacht werden.

In den univariaten Berechnungen konnten mehrere signifikante Zusammenhänge zu den weiteren Variablen aufgezeigt werden (Tabellen 23-31). Diese einzelnen Variablen mit signifikanten p-Werten wurden $\mathrm{zu}$ weiteren Berechnungen herangezogen.

Bezüglich des gesamten Patientenkollektivs ergab die logistische Regressionsanalyse folgende Ergebnisse:

Aus den Berechnungen der Variablen zu der Gesamtheit der Non-albicans-Isolate ist zu entnehmen, dass das Alter ( $p=0,0438$, in Verbindung zu den weiteren Variablen dieser Gruppe wie eigenständige Mundhygiene, Prothesen sowie deren Hygiene, etc.) einen grenzwertig signifikanten Zusammenhang zu der Verteilung der Nonalbicans-Isolate hat.

Die übrigen Variablen zeigten keine weiteren Zusammenhänge in der multivariaten Regressionsanalyse. (Tabelle 24)

Es konnten keine multivariat signifikanten Zusammenhänge zwischen dem Fehlen eines mikrobiologischen Befundes und den univariat signifikanten Variablen Alter, Gruppeneinteilung und eigenständige Mundhygiene, gefunden werden. (Tabellen 25)

In dem Modell der Regressionsanalyse konnten keine weiteren multivariaten Zusammenhänge der mikrobiologischen Befunde zu den in der univariaten Berechnung signifikanten Variablen aufgezeigt werden.

(Hierzu siehe Spalte „multivariat“ in den Tabellen 26-31) 


\section{Diskussion}

Symptome und Befunde von Haut und Schleimhäuten stellen gerade bei vulnerablen Patientengruppen eine häufige, relevante und dennoch unterschätzte klinische Problematik dar. Eine vorangegangene Pilot-Untersuchung der Klinik für Palliativmedizin an Patienten der Palliativstation bezüglich enoraler Symptome in der Palliativmedizin deutete sowohl auf eine hohe Prävalenz als auch klinische Relevanz von Candida bei diesen besonders schwer erkrankten Patienten hin (Alt-Epping et al. 2012). Daher wurden nun im Rahmen einer größeren Studie weitere Patientengruppen zu dieser Fragestellung untersucht. Einerseits Patienten auf einer Intensivstation, einer geriatrischen Station sowie einer Palliativstation im Rahmen einer gleichzeitig gestarteten Promotionsarbeit durch G. Ungermann sowie im Rahmen der hier vorliegenden Promotionsarbeit eine weitere, nicht grundsätzlich als krank geltende Zielgruppe (Bewohner/-innen stationärer Pflegeeinrichtungen sowie eines Demenzpflegeheimes) sowie eine Kohorte von Studierenden der Medizin als Kontrollgruppe. Alle Probanden wurden auf ihre enorale Symptomlast, auf klinische Befunde an Haut und Schleimhaut und (fokussiert) auf ihre mukokutane Candidabesiedlung hin untersucht (incl. Subgruppen- und Resistenztestungen). Dies wurde in Korrelation zu weiteren Pflege- und Umfeldfaktoren gesetzt.

Bei der Interpretation der Ergebnisse muss berücksichtigt werden, dass im Rahmen dieser Studie lediglich lokale Besiedlungen der Haut und Schleimhaut untersucht wurden, welche u.a. mit höherem Alter und/oder dem Tragen einer Zahnprothese in Verbindung gebracht werden konnten. Aufgrund der gewählten Methodik wurde keine invasive Mykose im Sinne einer Infektion nachgewiesen. Dies hätte sicher relevante klinische Konsequenzen im Sinne von therapeutischer Intervention nach sich gezogen. 


\subsection{Risikofaktor Alter / Resistenzlage}

Die hohe Prävalenz und Relevanz einer Candidabesiedlung wurde in der vorliegenden Studie erneut bestätigt - nicht nur bei Palliativpatienten, sondern bei allen getesteten Probanden im Krankenhaus (siehe ebenfalls die parallele Arbeit von G. Ungermann) und den stationären Pflegeeinrichtungen. Die Verteilung der Pilzspezies wies in den untersuchten Einrichtungen ein ähnliches Verteilungsmuster auf und zeigte, dass mit steigendem Alter C. glabrata an Häufigkeit zu- und $C$. albicans abnimmt.

Dadurch, dass sich das Spektrum der mykologischen Befunde bei älteren Menschen gleich darstellte, scheint die Pilzbesiedlung der Untersuchungsgruppe unabhängig von den untersuchten Komorbiditäten und den Grunderkrankungen der Probanden, aber abhängig vom Alter zu sein, wobei Non-albicans-Isolate einen signifikanten Zusammenhang mit einem höheren Lebensalter der Probanden zeigten.

Die Häufigkeit von C. albicans-Isolaten hingegen nahm mit zunehmendem Alter ab. Mit höherem Alter nahm die Häufigkeit mykologischer Befunde insgesamt zu. Das Alter konnte als einzige Variable in der logistischen Regressionsanalyse mit der Gruppe der Non-albicans-Isolate signifikant in Verbindung gebracht werden.

In Verbindung zu dem Altersunterschied der Probanden könnten jedoch auch weitere Komorbiditäten, welche in dieser Studie nicht eruiert wurden, die signifikant unterschiedliche Verteilung der Hefen zwischen Jung und Alt begünstigen.

Es bestand ein hochsignifikanter Zusammenhang zwischen dem Alter der Probanden und dem Nachweis einer Pilzbesiedlung sowie dem Tragen einer Zahnprothese und Non-albicans. Daher besteht die Vermutung, dass ein hohes Alter in Verbindung mit einer schlechteren manuellen Geschicklichkeit sowie dem Tragen einer Zahnprothese eine orale Pilzbesiedlung begünstigen könnte. Allerdings wurde nicht bei jedem älteren Probanden mit einer Prothese ein positiver Pilznachweis gefunden. Zu ähnlichen Ergebnissen kamen Malani et al. 2011 in einer Studie, in der gezeigt werden konnte, dass Candida glabrata ausschließlich bei älteren Probanden (>40Jahre) gefunden wurde und eng mit zunehmendem Alter, dem Tragen von Zahnprothesen sowie der Einnahme von Psychopharmaka einherging. 
Die Untersuchung zeigt, dass mit zunehmendem Alter die Prävalenz oraler Candidabesiedlung steigt (insbesondere Non-albicans-Spezies wie C. glabrata). Ursachen hierfür können möglicherweise in einer veränderten Immunabwehr wie bei immunsupprimierten Patienten (Ahariz et al. 2010) und/ oder den Abwehrmechanismen der Schleimhaut begründet sein, die es C. glabrata ermöglichen, die orale Schleimhaut zu kolonisieren und zunehmend zu dominieren, wodurch $C$. albicans stetig in den Hintergrund gedrängt wird.

Zudem beinhaltet diese Verschiebung in der Speziesverteilung im Alter weitere Besonderheiten. Die Therapie einer durch C. albicans hervorgerufen Mykose unterscheidet sich u.a. in der Auswahl des einzusetzenden Antimykotikums von der Therapie im Falle einer Infektion durch Non-albicans Hefen. C. albicans Hefepilze können effektiv mittels Azolderivaten oder auch Nystatin, teils auch in topischer Applikation, therapiert werden. C. glabrata hingegen zeigte jedoch im Laufe dieser Untersuchungen eine Vielzahl an Resistenzen gegenüber Azol-Antimykotika, welche die am häufigsten verschriebene Gruppe von Antimykotika repräsentieren. Nonalbicans Hefen wie C. glabrata zeigten sich gegenüber Polyenen wie Amphothericin B oder Nystatin empfindlich. Bereits 2010 empfahlen Richardson und Antilla (2010) einen zurückhaltenden Einsatz von Azolen im Falle von rezidivierenden Infektionen, um Resistenzen vorzubeugen.

Falls eine Indikation für die Behandlung der Candidose vorliegt, sollte daher das Alter des zu therapierenden Patienten berücksichtigt werden, um eine zunehmende Resistenzentwicklung der C. glabrata-Spezies oder anderer Non-albicans-Spezies zu vermeiden. Auch wenn $C$. albicans immer noch die am häufigsten vorzufindende Spezies in dieser Studie ist, so zeigte der mykologische Befund in Deutschland (Borg-von Zepelin et al. 2007) sowie weltweit (Pfaller und Diekema 2007) in vielen Fällen eine Kolonisation mit C. glabrata und nicht mit C. albicans.

Vergleicht man die in dieser Studie gefundenen Ergebnisse mit regionalen Untersuchungen zur Speziesverteilung (Borg-von Zepelin et al. 2007), zeigt sich, dass C. glabrata nicht nur regional in den untersuchten stationären Pflegeeinrichtungen eine Rolle zu spielen scheint, sondern sich überregional ähnliche Trends abzeichnen lassen. So konnten Pfaller und Diekema (2007) zeigen, dass sich in den USA ein ähnlicher Trend entwickelt hat, wobei die Anzahl von C. glabrataIsolaten bei invasiver Candidiasis zunimmt. Ebenso konnten Resistenzen gegenüber 
Triazolen und/ oder Amphotericin festgestellt werden. Hierdurch zeigen sich die klinische Relevanz der Untersuchung und die daraus resultierende Überlegung, dass bei älteren Menschen mit enoralen Symptomen vor einem Einsatz mit Azolderivaten zur Therapie der Mykosen eine mykologische Untersuchung bzgl. möglicher Resistenzen sinnvoll ist.

Im Zweifelsfall gibt eine Resistenztestung Aufschluss über die Medikation. Diese diagnostische Möglichkeit sollte bei der Auswahl der Antimykotika zukünftig berücksichtigt werden um erfolgversprechend zu therapieren.

\subsection{Statistische Ergebnisse}

\subsubsection{Orale Isolate}

Neben der Korrelation mikrobiologischer Befunde mit dem höheren Alter konnten in dieser Studie weitere signifikante Korrelationen zwischen mikrobiologischen Befunden, klinischen Aspekten und Umgebungsfaktoren ermittelt werden.

Klinische Befunde der Mundhöhle (wie z.B. Rötungen, Beläge) standen in Zusammenhang mit oraler Candidabesiedlung. Bei jüngeren Probanden konnte häufiger C. albicans, bei älteren Probanden zunehmend Non-albicans (insbesondere C. glabrata) nachgewiesen werden.

Insgesamt wurden jedoch nur sehr selten enorale Symptome von den Probanden der stationären Pflegeeinrichtungen beschrieben. Die größte Anzahl der Probanden beschrieb keine subjektiven Beschwerden, insofern unterschied sich das Bild der Symptomlast deutlich zur Pilotstudie, in der Probanden der Klinik für Palliativmedizin befragt und untersucht wurden (Kordestani 2009), obwohl eine ähnliche Häufigkeit mykologischer Befunde vorlag.

\subsubsection{Isolate der Leistenfalte}

Die Proben der Leistenfalte konnten in keiner Weise mit den klinischen Befunden in einen signifikanten Zusammenhang gebracht werden. Auch an diesem Abstrichort 
wurde angenommen, dass die Mehrzahl der Candida-Isolate in der Untersuchungsgruppe und nicht in der Kontrollgruppe gefunden wird. Es wurde vermutet, dass sich Candida-Isolate besonders an Körperregionen nachweisen lassen können, an denen Bereiche der Haut Kontakt haben bzw. wo sich Hautfalten bilden, welche ein feucht-warmes Milieu und somit das Wachstum von Candida begünstigen. Auch wenn sich bei älteren Probanden rein optisch aufgrund sichtbarer, vereinzelt rötlich-entzündlicher Hautveränderungen annehmen ließ, dass CandidaIsolate vorhanden sein könnten, fiel die Anzahl der Hefennachweise unerwartet niedrig aus. Zudem war die Summe positiver Abstriche in der Kontrollgruppe unerwartet höher als die der Untersuchungsgruppe. Hier könnte einerseits eine exzellente Körperpflege in den Pflegeeinrichtungen postuliert werden, andererseits wird es weiterer Anstrengungen (zum Beispiel in Form weiterer Kohortenuntersuchungen) bedürfen um zu erklären wieso in der Kontrollgruppe junger gesunder Probanden eine höhere Prävalenz von Candidabesiedlung gefunden wurde als bei Pflegeheimbewohnern/-innen.

\subsubsection{Isolate der Zehenzwischenräume}

Zeigte sich ein Zusammenhang zwischen der Candidabesiedlung mit zunehmendem Alter im oralen Bereich (s.o.), so konnte ebenfalls ein signifikanter Zusammenhang an den Zehenzwischenräumen jüngerer Probanden gefunden werden. Jedoch gibt es deutliche Unterschiede in der Speziesverteilung beider Abstrichorte: während die oralen Non-albicans-Isolate der älteren Probanden größtenteils von C. glabrata repräsentiert wurden, zeigte sich ein umgekehrtes Bild der Speziesverteilung an der Fußregion. Hierbei wurde anstatt $C$. glabrata eher $P$. guilliermondii und $C$. parapsilosis gefunden.

Auch bei den Abstrichen der Zehenzwischenräume zeigte sich der unerwartete Befund, dass jüngere Probanden der Kontrollgruppe häufiger einen positiven mykologischen Nachweis hatten als die älteren untersuchten Pflegeheimbewohner/innen und auch hier bedarf es noch einer plausiblen Erklärung, zum Beispiel aus weiterführenden Kohortenstudien. Untersuchungen die die hier vorliegenden Befunde untermauern konnten in der nationalen und internationalen Literatur so nicht gefunden werden. 
Eine mögliche Ursache für die deutlich höhere Anzahl an Pilzbesiedlungen des Zehenzwischenraumes der Kontrollgruppe kann darin begründet liegen, dass die Probennahme zu einer warmen Jahreszeit (Sommermonate) durchgeführt wurde in der das in der Klinik zu tragende geschlossenes Schuhwerk und höhere Temperaturen ein durch die physiologische Transpiration bedingtes feucht-warmes Milieu bewirken und damit einen stärkeren Pilzbefall erklären könnten.

In Pflegeheim 1 konnten keine Anzeichen einer Pilzbesiedlung an der Leistenfalte oder der Zehenzwischenräume gefunden werden. Dies lässt vermuten, dass die Hygiene dieser Bereiche äußerst erfolgreich zu sein scheint. Im Umkehrschluss kann jedoch nicht davon ausgegangen werden, dass die Hygienemaßnahmen anderer Pflegeeinrichtungen weniger effektiv sind, da Hefen ebenfalls als kommensale Keime in der physiologischen Flora vorhanden sein können, ohne eine Infektion zu verursachen.

Aufgrund der Fehlenden Beschwerdesymptomatik der wenigen mykologischen Befunde an Leistefalte und Fuß scheinen diese lokal begrenzten Hefenachweise eine klinisch untergeordnete Rolle zu spielen. Durch lokale antimykotische Maßnahmen könnten diese Pilzbesiedlungen bei klinischer Erfordernis auf ein Minimum reduziert werden. Hierbei ist jedoch durch gründlichere Hygiene der betroffenen Körperregionen (Leistenfalte oder Zehenzwischenräume) bereits ohne den Einsatz antimykotischer Maßnahmen mit einer Keimreduktion zu rechnen.

\subsection{Weitere Zusammenhänge}

Ob eine Besiedlung der Haut oder Schleimhaut bei veränderter Immunabwehr des Wirtes zu einer Infektion führen kann, gilt es weiterhin zu untersuchen. Hierbei wären angesichts der Untersuchungsergebnisse resistenzadaptierte prophylaktische Maßnahmen sinnvoll. Bei der empirischen Auswahl des einzusetzenden Antimykotikums können die gefundenen Korrelationen, insbesondere zum Alter des Patienten, eine wichtige Hilfe darstellen. Im Tiermodell konnten allerdings bisher 
keine Besiedlung der Haut als Ursache für eine invasive Mykose festgestellt werden (Sohnle und Hahn 1989).

\subsubsection{Hygienemaßnahmen/ Zahnprothesen als Risikofaktoren}

Das insgesamt häufigere Auftreten eines mikrobiologischen Befundes könnte vermutlich in einer unzureichende Mundhygiene sowie geringerer manueller Geschicklichkeit begründet liegen. Der signifikante Zusammenhang zwischen Pilzbefall und dem Tragen von Zahnprothesen lässt ebenfalls vermuten, dass die tägliche Reinigung des Zahnersatzes sowie eine richtig durchgeführte Mundhygiene bereits zu einer Reduktion oraler Hefen beitragen könnten.

Hierzu wurden bereits Untersuchungen durchgeführt (Yonezawa et al. 2003), deren Ansätze zu einer deutlichen Keimreduktion der Hefen auf Zungenrücken und Schleimhaut führen können. Ebenfalls konnte gezeigt werden, dass bereits der Gebrauch einer Zahnbürste zur täglichen Mundhygiene eine deutliche Keimreduktion zur Folge hatte. Zur Prävention oraler Pilzbesiedlungen scheint diese einfache Anwendung bereits zu guten Ergebnissen zu führen.

In jedem Fall muss laut Richardson und Antilla (2010) der orale Biofilm zusätzlich mechanisch aufgebrochen werden, um erfolgversprechend die Erregerzahl herabzusetzen.

Des Weiteren kann das Tragen einer Zahnprothese bzw. deren unzureichende Reinigung eine Kolonisation durch Hefen begünstigen (Kulak-Ozkan et al. 2002; Nishiyama et al. 2010).

Gerade bei Zahnprothesenträgern und protheseninduzierter Stomatitis konnte bereits in früheren Studien C. albicans als häufigste sowie C. glabrata als zweithäufigste Spezies nachgewiesen werden (Coco et al. 2008).

Da Non-albicans-Hefen häufig bei Prothesenträgern gefunden wurden könnten Adhäsionsvorgänge von Non-albicans-Isolaten (insbesondere C. glabrata als häufigster Vertreter) an Zahnprothesen einen möglichen Rückschluss auf den hohen Pilzbefall bei Prothesenträgern geben.

Da von dem oralen Befund bzw. den Symptomen einer oralen Candidose nicht direkt auf die Erregersubspezies geschlossen werden kann sind mikrobiologische Speziesdifferenzierungen, wie bereits oben erwähnt, für eine gezielte antimykotische Therapie hilfreich. 
Inwieweit sich die Adhäsion der Hefepilze an Methacrylaten der Prothesenbasenoberfläche unterscheiden und ob dies eine zunehmende Besiedlung durch Candida (speziell C. glabrata) ermöglicht, gilt es weiterhin zu untersuchen.

Es wurde bei Probanden, die angaben, zusätzliche Mundhygieneartikel zu verwenden, eine höhere Anzahl von Isolaten nachgewiesen als bei Probanden ohne weitere Mundhygieneartikel (Tabelle 24).

Da jedoch aus dem Fragebogen der Kontrollgruppe nicht hervorgeht ob und wenn ja welche Mundhygieneartikel verwendet wurden spiegelt diese prozentuale Verteilung nicht die tatsächliche Situation der gesamten Probandengruppe wider.

Die Berechnung zu Pilzbefall vs. Mundhygiene-Artikel ohne die Daten der Kontrollgruppe zeigte keine statistische Signifikanz.

Daher ist anzunehmen, dass kein Zusammenhang zwischen der Verwendung von Mundhygiene-Artikeln und einem Pilznachweis besteht.

Eine ähnliche Berechnungsproblematik zeigte sich bei der Frage nach der Häufigkeit der Prothesenhygiene.

Ein signifikanter Zusammenhang konnte zwischen der Prothesenhygiene und dem Auftreten von Non-albicans-Isolaten festgestellt werden (Tabelle 24).

$\mathrm{Da}$ in der Kontrollgruppe aufgrund des jungen Alters keine Zahnprothesenträger vorhanden sind, muss dies bei der Wertung des Ergebnisses berücksichtigt werden.

Da die jüngeren Probanden die Verteilung zugunsten des nicht-prothesentragenden Anteils und somit auch nicht-prothesenpflegenden Anteils verschieben, kann immer noch davon ausgegangen werden, dass eine durchgeführte Prothesenhygiene zu einer Keimreduktion beiträgt.

Die alleinige Berechnung zu der Prothesenhygiene der Bewohner der stationären Pflegeeinrichtungen zeigte keine statistische Signifikanz.

\subsubsection{Mögliche Folgestudien}

Des Weiteren können in Folgestudien Aussagen zu mikrobiologischen Befunden wie Dermatophyten und Schimmelpilzen dieser Probandengruppe gemacht werden, welche ebenfalls mit untersucht wurden, jedoch in dieser Arbeit nicht berücksichtigt werden konnten. Hierzu wurden gleichermaßen wie bei o. g. Hefen verfahren. 
Kulturen wurden angelegt, beobachtet, dokumentiert und für spätere Verwendungen aufbewahrt. Ebenfalls wurde parallel zu den o. g. Hefen eine Speziesdifferenzierung der Schimmelpilze und Dermatophyten durchgeführt.

Eine Analyse der Daten hierzu ging jedoch deutlich über das Maß dieser Arbeit hinaus.

Die Kulturen der Schimmelpilze und Dermatophyten sowie die Ergebnisse der Speziesdifferenzierung können in einer weiteren Arbeit ausgewertet werden.

\subsection{Limitationen}

Diese Untersuchung weist eine Zahl an Limitationen auf:

\subsubsection{Befunderhebungsbögen}

Der Befunderhebungsbogen der Kontrollgruppe war im Vergleich zu den Probanden der stationären Pflegeeinrichtungen deutlich verkürzt. Dadurch wurden in der Kontrollgruppe Symptome und klinische Befunde nicht in derselben Ausführlichkeit erfasst. Ein kürzerer Fragebogen wurde im Vorfeld für die Kontrollgruppe gewählt, da primär der mikrobiologische Befund im Vordergrund stand.

\subsubsection{Rekrutierung der Probanden/ Compliance}

Insbesondere mit Blick auf das teils aufwändige informed-consent-Procedere blieb die Zahl der rekrutierten Patienten in den stationären Pflegeeinrichtungen unter der im Voraus avisierten Anzahl. Dennoch konnten in vielen Bereichen entsprechende klinisch relevante Signifikanzen nachgewiesen werden. Die Anzahl der Pflegeheimbewohner/-innen war begrenzt. Nachdem die gesamte Anzahl der Bewohner/-innen der stationären Pflegeeinrichtungen um eine Teilnahme gebeten worden war, konnte das Feld der Probanden nicht weiter aufgefüllt werden.

Es zeigte sich, dass häufiger eine Zustimmung zur Befragung, Untersuchung und Probenentnahme gegeben wurde, wenn die Bewohner/-innen (und deren Betreuer) zusätzlich zu den Unterlagen der Studie schriftlich von dem betreffenden Pflegeheim 
über die Ziele der Studie unterrichtet wurden. Hierdurch signalisierte die Pflegeheimleitung die Unterstützung des Vorhabens, welches die Berührungsängste zur Untersuchung -die zwar nicht-invasiv war, jedoch eine Unbekannte darstelltedeutlich zu senken schien.

Die Compliance war bei dem Großteil der Probanden sehr gut, was in vielen Fällen auf die Anwesenheit eines Mitarbeiters der jeweiligen stationären Pflegeeinrichtung sowie deren Hilfestellung zurückzuführen war.

Während der Datenerhebung verweigerten nur äußerst selten Probanden nachträglich die Teilnahme an der Studie.

\subsection{Rückschlüsse auf die aktuelle Pflegesituation}

Aus den Ergebnissen dieser Studie geht hervor, dass die Prävalenz und klinische Relevanz oraler Schleimhautbesiedlungen durch Hefen insbesondere bei zunehmendem Alter einen nicht zu unterschätzenden Platz einnimmt und in der täglichen Pflege präsent ist.

Es konnte jedoch auch gezeigt werden, dass nicht jede orale Pilzbesiedlung mit klinischen Symptomen und Beschwerden einhergeht und daher selten eine direkte Indikation zur antimykotischen Therapie bei einem gesicherten Pilznachweis besteht. Eingedenk der Verteilung der Non-albicans-Hefen und der damit verbundenen auffälligen Resistenzlage stellen diese Ergebnisse jedoch eine wichtige Information zum Beispiel bei immunsupprimierten Patienten und der damit verbundenen Gefahr der Erregerausbreitung dar.

Weitestgehend vergleichbar ist diese Feststellung aus den Ergebnissen dieser Studie mit einer Infektion durch das Varizella-Zoster-Virus aus der Familie der Herpesviren. Hierbei sind die Viren nach einer erstmaligen Infektion (Windpocken) lebenslang latent vorhanden. Eine antivirale Therapie ist indiziert sobald Symptome (wie zum Beispiel Schmerzen oder Hauteffloreszenzen entlang des segmentalen Ausbreitungsgebietes der Nervenfasern) auftreten. Diese Reaktivierung tritt besonders in Phasen auf, welche durch Stress oder ein geschwächtes Immunsystem gekennzeichnet sind. Analog zu der Therapie des Varizella-Zoster-Virus mit antiviralen Substanzen ist auch der richtige Zeitpunkt der antimykotischen Therapie vulnerabler Patientengruppen relevant. 
Ein Vorhandensein von klinischen Befunden konnte signifikant mit dem Vorhandensein von Hefen in Verbindung gebracht werden. Weitere Untersuchungen wie eine Abstrichentnahme sowie Speziesdifferenzierung sind nötig, da das (intraorale) Erscheinungsbild keinen Rückschluss auf die Spezies zulässt.

Häufig waren Pilzbesiedlungen jedoch ohne eine Beschwerdesymptomatik vorhanden.

Eine Inspektion scheint daher verlässlich zu sein, um eine Pilzbesiedlung zu diagnostizieren.

Kontrollen durch betreuende Ärzte/ Zahnärzte und ebenfalls des Pflegepersonals sind daher essentiell für ein frühes Erkennen soortypischer Mundschleimhautveränderungen.

Wie bereits oben erwähnt muss eine Pilzbesiedlung jedoch nicht zwangsläufig mit einer Therapie einhergehen.

Zu welchen Zeitpunkt Therapiemaßnahmen indiziert sind (Z.B. bei dem Auftreten von Symptomen) geht aus den vorliegenden Daten nicht hervor.

Vermutlich lassen sich subjektive Beschwerden (welche in dieser Studie im Gegensatz zur Pilotstudie äußerst selten waren) erst zu einem späten Zeitpunkt im Verlauf der Erkrankung eruieren. Eine regelmäßige Kontrolle bzw. Inspektion könnte jedoch bei einer frühzeitigen Feststellung von Symptomen mit Anzeichen von oralen Mykosen durch lokale antimykotische Maßnahmen die Häufigkeit einer Beschwerdesymptomatik senken. 


\section{$5 \quad$ Zusammenfassung}

Es wurden in der vorliegenden Studie Bewohner/-innen aus insgesamt drei verschiedenen stationären Pflegeeinrichtungen (teils mit explizitem Demenzpflegeschwerpunkt) sowie eine Kontrollgruppe jüngeren Alters auf mukokutane Symptome und Pilzbesiedlungen untersucht.

Ein positiver mykologischer Befund stand in deutlichem Zusammenhang mit einem höheren Alter, der Pflegestufe, dem Gebrauch von Zahnprothesen, einer unzureichenden Prothesenhygiene, einer fehlenden Eigenständigkeit bei der Mundhygiene sowie einem klinisch auffälligem Befund der Mundhöhle. Diese o. g. Kriterien zeigten einen signifikanten Zusammenhang insbesondere zu Non-albicansSpezies.

Die mit Abstand größte Anzahl an Pilznachweisen wurde aus den Isolaten der Mundhöhle gewonnen.

Ältere Bewohner/-innen der stationären Pflegeeinrichtungen zeigen eine stärkere Besiedlung der Schleimhäute mit Hefesporen als jüngere Probanden.

Non-albicans-Spezies mit einer Resistenz gegenüber Azolderivaten waren bei älteren Personen häufiger vorzufinden. Besonders im Hinblick auf die mit dem Alter zunehmende Anzahl von Prothesenträgern sowie eine zunehmend eingeschränkten eigenständigen Mundhygiene sollte die unkritische Anwendung von AzolAntimykotika bei Personen höheren Alters ohne vorherige Speziesanalyse und Suszeptibilitätsprüfung vermieden werden.

C. albicans wurde in den Proben der Kontrollgruppe (jüngerer Probanden) häufiger isoliert als Non-albicans-Spezies.

Abstriche der Leistenfalte zeigten keine signifikanten Korrelationen zu weiteren Faktoren auf.

Das jüngere Alter (und damit Probanden der Kontrollgruppe) korrelierte statistisch mit dem mikrobiologischem Befund der Zehenzwischenräume. Literatur im nationalen und internationalen Bereich, mit der man diese Befunde untermauern könnte, fehlt bisher. 
Die Auswertung der deskriptiven Daten zu weiteren Symptomen und Angaben der Probanden (Geschlecht, Diabetes mellitus, Bestrahlung, Chemotherapie, Immunsupressiva, Antibiotika, Xerostomie, eigenständige Mundhygiene, Zahnprothese, Speichelsekretion, Hauttyp) zeigten hingegen keine weiteren statistisch signifikanten Zusammenhänge zu den mikrobiologischen Befunden.

Bei der Interpretation der Befunde ist zu berücksichtigen, dass sich alle ermittelten Daten auf den Nachweis von Hefen beziehen, die bei der Abstrichuntersuchung der Schleimhäute des Mundes sowie der Leistenfalte oder der Zehenzwischenräume erhoben wurden, nicht jedoch auf eine Infektion mit demselben Erreger. Die genaue Anzahl der Kolonien wurde nicht berücksichtigt. Für einen möglichen prophylaktischen Einsatz von Antimykotika sind daher weitere Analysen notwendig. 


\section{$6 \quad$ Literaturverzeichnis}

Ahariz M, Loeb I, Courtois P (2010): Oral candidiasis and dentures. Rev Stomatol Chir Maxillofac 111, 74-78

Albougy HA, Naidoo S (2002): A systematic review of the management of oral candidiasis associated with HIVIAIDS. SADJ $\underline{57}$, 457-466

Alt-Epping B, Nejad RK, Jung K, Groß U, Nauck F (2012): Symptoms of the oral cavity and their association with local microbiological and clinical findings - a prospective survey in palliative care. Support Care Cancer 20, 531-537

Bagg J, Sweeney MP, Lewis, M A O, Jackson MS, Coleman D, AI MA, Baxter W, McEndrick S, McHugh S (2003): High prevalence of non-albicans yeasts and detection of anti-fungal resistance in the oral flora of patients with advanced cancer. Palliat Med 17, 477-481

Borg-von Zepelin M, Kunz L, Ruchel R, Reichard U, Weig M, Gross U (2007): Epidemiology and antifungal susceptibilities of Candida spp. to six antifungal agents: results from a surveillance study on fungaemia in Germany from July 2004 to August 2005. J Antimicrob Chemother $\underline{60}, 424-428$

Cassone A, Cauda R (2012): Candida and candidiasis in HIV-infected patients: where commensalism, opportunistic behavior and frank pathogenicity lose their borders. AIDS $\underline{26}, 1457-1472$

Chen YC, Eisner JD, Kattar MM, Rassoulian-Barrett SL, LaFe K, Yarfitz SL, Limaye AP, Cookson BT (2000): Identification of medically important yeasts using PCRbased detection of DNA sequence polymorphisms in the internal transcribed spacer 2 region of the rRNA genes. J Clin Microbiol 38, 2302-2310

Coco BJ, Bagg J, Cross LJ, Jose A, Cross J, Ramage G (2008): Mixed Candida albicans and Candida glabrata populations associated with the pathogenesis of denture stomatitis. Oral Microbiol Immunol 23, 377-383

Dorko E, Jenca A, Pilipcinec E, Danko J, Svicky E, Tkacikova L (2001): Candidaassociated denture stomatitis. Folia Microbiol (Praha) 46, 443-446

Glazar I, Urek MM, Brumini G, Pezelj-Ribaric S (2010): Oral sensorial complaints, salivary flow rate and mucosal lesions in the institutionalized elderly. J Oral Rehabil $\underline{37}, 93-99$ 
Hof H (2010): Mycoses in the elderly. Eur J Clin Microbiol Infect Dis 29, 5-13

Krishnan PA (2012): Fungal infections of the oral mucosa. Indian J Dent Res $\underline{23}$, 650-659

Kordestani R: Enorale Symptome in der Palliativmedizin. Med. Diss. Göttingen 2009

Kulak-Ozkan Y, Kazazoglu E, Arikan A (2002): Oral hygiene habits, denture cleanliness, presence of yeasts and stomatitis in elderly people. J Oral Rehabil $\underline{29}$, 300-304

Laurent M, Gogly B, Tahmasebi F, Paillaud E (2011): Oropharyngeal candidiasis in elderly patients. Geriatr Psychol Neuropsychiatr Vieil $\underline{9}$, 21-28

Malani AN, Psarros G, Malani PN, Kauffman CA (2011): Is age a risk factor for Candida glabrata colonisation? Mycoses $\underline{54}, 531-537$

Martinez Machin G, Perurena Lancha M, Nunez Carvajal J, Fernandez Andreu, C M, Bandera Tirado F (1997): Isolation, identification, and typing of yeasts from HIVpositive patients with oral candidiasis. Rev Cubana Med Trop 49, 174-180

Nishiyama Y, Inaba E, Uematsu H, Senpuku H (2010): Effects of mucosal care on oral pathogens in professional oral hygiene to the elderly. Arch Gerontol Geriatr $\underline{51}$, e139-43

Peman J, Salavert M (2012): General epidemiology of invasive fungal disease. Enferm Infecc Microbiol Clin 30, 90-98

Pfaller MA, Diekema DJ (2007): Epidemiology of invasive candidiasis: a persistent public health problem. Clin Microbiol Rev 20, 133-163

Richardson R, Antilla V (2010): Diagnosis and treatment of oral candidosis. Duodecim $\underline{126}, 174-180$

Rodriguez-Tudela JL, Arendrup MC, Barchiesi F, Bille J, Chryssanthou E, CuencaEstrella M, Dannaoui E, Denning DW, Donnelly JP, Dromer F (2008): EUCAST Definitive Document EDef 7.1: method for the determination of broth dilution MICs of antifungal agents for fermentative yeasts. Clin Microbiol Infect 14, 398-405

Sohnle PG, Hahn BL (1989): Effect of immunosuppression on epidermal defenses in a murine model of cutaneous candidiasis. J Lab Clin Med 113, 700-707

Sweeney MP, Bagg J (2000): The mouth and palliative care. Am J Hosp Palliat Care 17, 118-124

Yamamoto T (2010): Oral candidiasis: clinical features and control. Rinsho Byori $\underline{58}$, 1027-1034 
Yonezawa H, Takasaki K, Teraoka K, Asaka T, Sato C, Tsuchiya K (2003): Effects of tongue and oral mucosa cleaning on oral Candida species and production of volatile sulfur compounds in the elderly in a nursing home. J Med Dent Sci $\underline{50}, 1-8$ 


\section{$7 \quad$ Abbildungsverzeichnis}

Abbildung 1: Altersverteilung der Untersuchungsgruppe.............................................. 21

Abbildung 2: Geschlechterverteilung nach Altersgruppen.................................................. 23

Abbildung 3: Verteilung der Isolate auf die Abstrichorte................................................ 34

Abbildung 4: Absolute Anzahl der Isolate aus Untersuchungs- und Kontrollgruppe

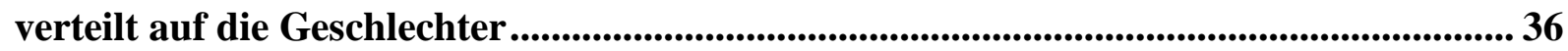

Abbildung 5: Prozentualer Vergleich der Isolate beider Geschlechter bezogen auf die männlichen/weiblichen Probanden der betreffenden Altersgruppe ................................. 37

Abbildung 6: Speziesverteilung aller Gruppen ........................................................................ 39

Abbildung 7: Häufigkeit der Isolate bezogen auf die Abstrichorte ................................ 42

Abbildung 8: Verteilung der Spezies auf die Altersgruppen .......................................... 43

Abbildung 9: Speziesverteilung verteilt auf die Altersgruppen (incl. „kein Wachstum“)44

Abbildung 10: Speziesverteilung bezogen auf die Altersgruppen .................................... 45

Abbildung 11: Daten der Resistenztestung: Amphothericin B.......................................... 46

Abbildung 12: Daten der Resistenztestung: Nystatin ....................................................... 46

Abbildung 13: Daten der Resistenztestung: Fluconazol................................................. 47

Abbildung 14: Daten der Resistenztestung: Voriconazol................................................ 47

Abbildung 15: Daten der Resistenztestung: Micafungin ......................................................... 48 


\section{$8 \quad$ Tabellenverzeichnis}

Tabelle 1: Verbrauchsmaterialien ......................................................................................... 17

Tabelle 2: Antimykotika ....................................................................................................................... 18

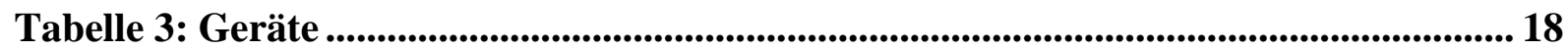

Tabelle 4: Verteilung der Probanden nach Alter und Geschlecht................................... 22

Tabelle 5: Einteilung nach Altersgruppen .................................................................................... 22

Tabelle 6: Kontrollgruppe (Anzahl und durchschnittliches Alter) ...................................... 23

Tabelle 7: Ergebnisse der Befunderhebungsbögen (1) Klinische Merkmale..................... 25

Tabelle 8: Ergebnisse der Befunderhebungsbögen (2) ADL ........................................... 26

Tabelle 9: Ergebnisse der Befunderhebungsbögen (3) Hygiene/ Prothetische Versorgung27

Tabelle 10: Ergebnisse der Befunderhebungsbögen (4) Verdauung / Hauttyp................. 28

Tabelle 11: Ergebnisse der Befunderhebungsbögen (5) Symptombelastung ..................... 29

Tabelle 12: Ergebnisse der Befunderhebungsbögen (6) Befund Mundhöhle.................... 30

Tabelle 13: Ergebnisse der Befunderhebungsbögen (7) Hautbefunde (1) ........................ 31

Tabelle 14: Ergebnisse der Befunderhebungsbögen (7) Hautbefunde (2) ........................ 32

Tabelle 15: Candidabesiedlung aller Probanden der Untersuchungsgruppe (80)........... 33

Tabelle 16: Anzahl Spezies verteilt auf beide Gruppen......................................................... 35

Tabelle 17: Prozentualer Vergleich beider Gruppen bezogen auf Abstrichort ................ 36

Tabelle 18: Verteilung der Candidabesiedlung innerhalb der Gruppen .......................... 38

Tabelle 19: Geschlechterspezifische Candidabesiedlung (Kontrollgruppe) ..................... 39

Tabelle 20: Anzahl der Non-albicans-Isolate Vergleich Pflegeheime vs. Kontrollgruppe41

Tabelle 21: Speziesverteilung der Gruppen (incl. Abstrichort) .......................................... 41

Tabelle 22: Verteilung der Probanden- und Isolate-Zahl auf die Altersgruppen............. 43

Tabelle 23: Gesamtheit der Isolate: C. albicans ................................................................50 
Tabelle 24: Gesamtheit der Isolate: Non-albicans ............................................................. 51

Tabelle 25: Gesamtheit der Isolate: kein klinisch auffälliger Befund ................................ 52

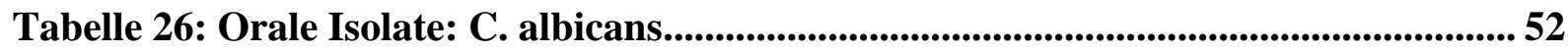

Tabelle 27: Orale Isolate: Non-albicans ....................................................................................... 53

Tabelle 28: Prothese und sonstige MH-Artikel: Untersuchungsgruppe ............................. 54

Tabelle 29: Orale Isolate: kein klinisch auffälliger Befund............................................... 55

Tabelle 30: Isolate Zehenzwischenraum: Non-albicans ...............................................56

Tabelle 31: Isolate Zehenzwischenraum: kein klinisch auffälliger Befund ...................... 57 


\section{$9 \quad$ Anhang}

\subsection{Ethikvotum}

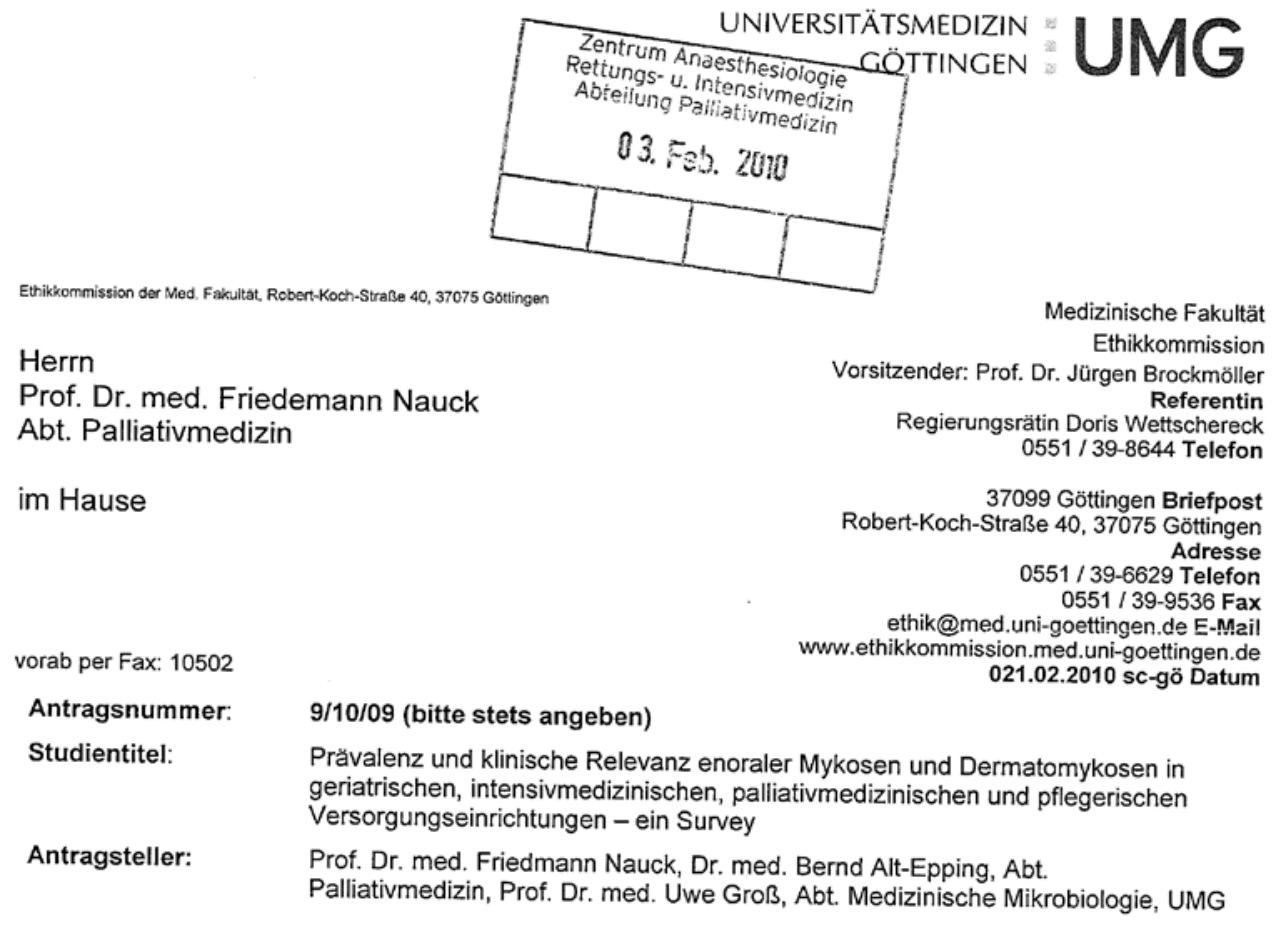

Sehr geehrter Herr Prof. Dr. Nauck, sehr geehrter Herr Dr. Alt-Epping, sehr geehrte Damen und Herren,

nach Ergänzung der vorliegenden Dokumente und Beantwortung der im vorläufigen Votum aufgeführten Fragen bestehen nunmehr keine ethischen und rechtlichen Bedenken gegen die Durchführung des oben genannten Forschungsvorhabens.

Wir wünschen viel Erfolg bei der Durchführung Ihres Projektes.

Auf folgende Punkte möchten wir hinweisen:

- Bitte ergänzen Sie auf S. 1 der Informationsschrift für Betreuer Satz 2 des 2. Absatzes folgendermaßen: „Ein Mitarbeiter der Universität möchte der von Ihnen betreuten Person (in deren Zimmer) die Mundschleimhaut....".

- Bei der Einverständniserklärung der Studenten muss auch der Satz „Ihre Daten sind vor ..." optisch hervorgehoben werden.

Bitte reichen Sie uns das ausgefüllte "Formblatt zur Aufwandserstattung" ein.

Die Ethik-Kommission weist darauf hin, dass die ärztliche und juristische Verantwortung bei den jeweiligen Prüfärzten verbleibt.

Auf die Einhaltung einschlägiger Gesetze und Rechtsvorschriften wird hingewiesen. Die nach Rechtslage notwendigen Unterrichtungen (u. A. Prüfplanänderungen, entsprechende Zwischenfallsereignisse, neue Datenlage, Nachmeldung von Prüfzentren, Abschlussbericht) sind der Ethik-Kommission unverzüglich vorzulegen.

Die Ethik-Kommission bestätigt, dass sie auf Grundlage nationaler Gesetze, Vorschriften sowie der GCP/ICH-Richtlinie arbeitet.

Mit freundlichen Grüßen

i. A.

$$
\text { Sching }
$$

Prof. Dr. med. J. Brockmöller

Vorsitzender der Ethik-Kommission

Universiț̌tsmedizin Göttinģen, Georg-August-Universităt Stiftung Offentlichen Rechts Vorstand Prot. Dr. Cornelius Frömmel (Forschung̣ \& Lehre, Sprecher des Vorstanđs) Prof. Or. Wolfgang Brück (komm. Krankenversorgung) Dipl.-Kffr. (FH) Barbara Schulte (Wirtschaftstürung \& Administration) Sparkasse Gzttingen (26050 500 ) kto 448 


\subsection{Befunderhebungsbogen der Bewohner/-innen der Pflegeeinrichtungen}

Befundbogennr

Datum:

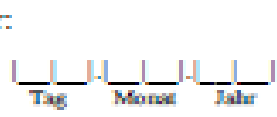

\section{UNIVERSITÄTSMEDIZIN $=\mathbf{J M G}$
GOTTINGEN $=\mathbf{M G}$}

\section{Befunderhebungsbogen}

Enorale Mykosen und Dermatomykosen bei Patienten in geriatrischen, intensivmedizinischen. palliativmedizinischen und pflegerischen Versorgungseinrichnugen

1. Geburtsdatum: L_L_- Monat Jahr Geschlecht: $\square$ weiblich $\square$ männlich

2. Hauptdiagnose:

Nebendiagnosen:

Diabetes mellirus / Typ / Behandlungsform

3. Ernahrung:

$\square$ oral (Kostform $\square$ enteral (Sonde)

$\square$ parenteral

4. Vorbehandlungen mit Auswirkungen auf den derzeitigen Gesundheitszustand-

$\square$ Operationen

$\square$ Bestrahlung, wenn ja Region und Dosis?: RegionDosis: L_ Gy

$\square$ Chemotherapie:

$\square$ Andere Therapiemabnahmen:

5. Alvtuelle Medikation:

Antibiotika: $\quad \square_{\text {nein }} \square_{\text {ja }}$

Immunsupressiva: $\square$ nein $\square$ ja; Substanzgruppe:

Antimykotika: $\square$ nein $\square$ ja

Medikamente mit Auswirkungen auf Speichelfluss und Nahrungsaufnahme: $\square$ nein $\square$ ja

Sonstige Medilkation: 
Befundbogennr:

Datum:

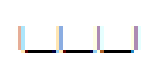

UNIVERSITÄTSMEDIZIN $=\mathbf{O M G}$
GOTTINGEN $=\mathbf{M G}$

6. Symptomatils:

$\square$ Keine Beschwerden $\square$ Xerostomie $\square$ Zahnschmerzen $\square$ Mundgeruch

$\square$ Unspezifisches Brennen $\square$ Dysgeusie $\square$ Dysphagie $\square$ Hautjucken

$\square$ trockene Haut $\square$ Spannungsgefuihl d. Haut

$\square$ Sonstiges

$\square$ Patient ist nicht befragbar: Grund:

7. Allgemeinzustand und Pflegebedarf ADL

Karnofsky Performace Score _-_

Pflegestufe $0 \square \quad 1 \square \quad 2 \square \quad 3 \square$

8. Mundhygiene

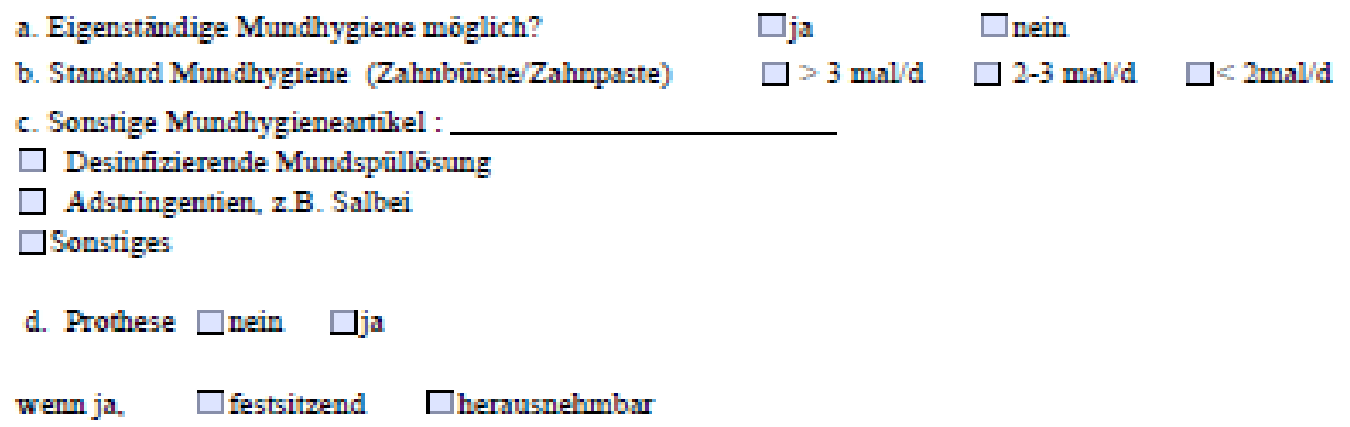

9. Klinischer Untersuchungsbefund Mundhōhle
$\square$ normal
$\square$ gerōtet
$\square$ geschwollen
$\square$ soortypische Belege
$\square$ anderweitige Belege
$\square$ Schleimhautveränderungen
$\square$ Ulcus
$\square$ Blutang akut
Aphte

$\square$ Sonstige relevante Befunde der Mundhöhle:

Speichelselkretion $\square$ normal $\square$ reduziert $\square$ vermehrt 
Befundbogenn:

Datum:



UNIVERSITÄTSMEDIZIN $=\mathbf{M M G}$
GOTTINGEN $=0$

12. Verdauung / Ausscheidungen

Durchfall in den letzten 4 wochen: $\square$ nein $\square$ ja

Wenn ja, wann? $\square$ aktuell $\square$ vor ca. 3 Tagen $\square$ vor 3 - 7 Tagen $\square$ vor 7 -14 Tagen $\square>14$ Tage

Wenn ja, Hāufigkeit / Tag:

13. Haut

a. Hautryp

$\square$ normal $\square$ trocken $\square$ fetrig $\square$ Mischhaut

b. Auffalligkeiten im Abstrichareal:

$\square$ keine $\square$ Rōtung $\square$ Schwellung $\square$ offene Wunde $\square$ Schuppen $\square$ Sonstige:

c. Sonstige Auffalligkeiten:

$\square$ keine $\square$ Rōoung $\square$ Schwellung $\square$ offene Wunde $\square$ Schuppen $\square$ Sonstige:

14. Fussnagel

Eigenständige Fussnagelpflege möglich? $\square$ ja $\square$ nein

Wenn ja, wie oft?

Wenn nein, wie oft FuBnagelpflege mit fremder Hilfe?

Fussnagelstatus: $\square$ unauffallig $\square$ ungepflegt $\square$ Entzīndungsreaktion $\square$ verfärbt

$\square$ sonstige klinische Befunde: 


\subsection{Befunderhebungsbogen der Kontrollgruppe}

Befundbogennr.

Datum:

$\frac{\mathrm{L}}{\text { Tag }} \cdot \frac{\mathrm{L}}{\text { Monat }} \cdot \frac{\mathrm{H}}{\text { Jahr }}$

\section{UNIVERSITARTSMEDIZIN
GÖTIINGEN $: \bigcup M M G$}

\section{Befunderhebungsbogen (Kontrollgruppe)}

Enorale Mykosen und Dermatomylkosen bei Patienten in geriatrischen, intensivmedizinischen, palliativmedizinischen und pflegerischen Versorgungseimrichtungen

1. Geschlecht

weiblich

mainnlich

2. Selbsteinschätrung ...Wie schãtzen Sie selbst Thre Mundhygiene ein?

$\square$ sehr gute Mundhygiene $\square$ gute Mundhygiene $\square$ mälige Mundhygiene

3. Mundhygienemittel-

Standard Munclhygiene (Zahnbürste/Zahnpaste) $\quad \square>3 \mathrm{mal} / \mathrm{d} \quad \square 2-3 \mathrm{mal} / \mathrm{d} \quad \square<2 \mathrm{mal} / \mathrm{d}$

4. Klinischer Befund Mundhōble

$\square$ Makroskopische Auffalligheit

$\square$ Keine makroskopische Auffalligkeit

5. Hautstatus

$\square$ Makroskopische Auffalligkeit

$\square$ Keine makroskopische Auffalligkeit

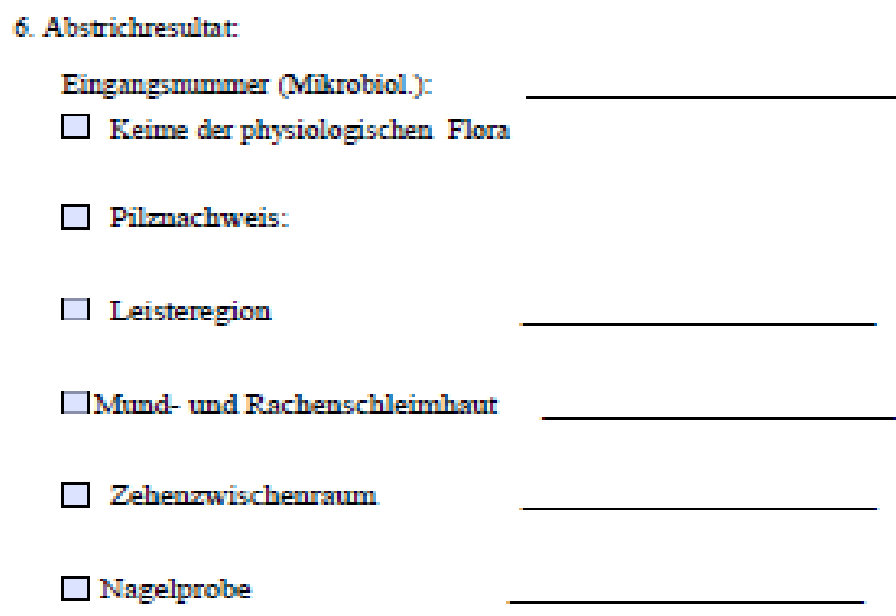




\subsection{Patienteninformationen (Probanden, Betreuer und Kontrollgruppe)}

\section{Patienteninformation}

Sehr geehrte Damen und Herren,

wir möchten Sie informieren über eine wissenschaftliche Studie unserer Klinik zum Thema

Symptome von Haut und Schleimhäuten

und ihre zugrundeliegenden mikrobiologischen Befunde

(Pravalenz und Reievanz enovaler Mykosen und Dermatomykosen in gerlatrischen, Intensivmedizin/schen, pallativmedizinischen und pflegerischen Versorgungseinrichtungen-ein Survey).

In der Behandlung schwerstkranker und betagter Patienten stehen die Wiederherstellung und der Erhalt der Lebensqualität ganz im Vordergrund der therapeutischen Bemūhungen. Beschwerden im Mund und Rachenraum und auf der Haut sind als häufiges, belastendes Problem anzutreffen. Bislang ist jedoch wenig bekannt über das tatsächliche Ausmaß dieser Problematik; es gibt Hinweise auf eine eventuell zugrundeliegende Besiedlung oder Infektion mit Pilzerregern, deren Behandlung zu einer effektiveren Linderung der Beschwerden führen könnte.

Daher möchten wir Sie um Ihre Teilnahme an einer freiwilligen wissenschaftlichen Studie bitten, die etwaige Beschwerden in Mund- und Rachenraum und auf der Haut und Ihre gesundheitlichen Situation genauer erfragt. Ein Mitarbeiter der Universität möchte Ihnen (in Ihrem Zimmer) die Mundschleimhaut und die Haut der Leistenregion und zwischen den Zehen mit einem Wattetupfer abstreichen. Zudem wird von Ihnen ein kleines Stück Fußnagel und eine Stuhlprobe erbeten. Falls Sie derzeit Patient auf der Intensivstation sind und Fieber haben, soll eine Blutkultur entnommen werden. Diese Proben werden in der Universitätsmedizin Göttingen auf eventuell vorhandene Erreger untersucht und anschließend vernichtet.

Die Untersuchung ist selbstverständlich freiwillig: bei Nichtteilnahme oder Widerruf 


\section{UNIVERSITATSMEDIZIN $=\mathbf{U M G}$
GOTTINGEN $=\mathbf{M G}$}

entstehen Ihnen keinerlei Nachteile. Die Untersuchung ist zudem schmerzfiei und ohne gesundheitliche Risiken. Die Untersuchungsergebnisse werden in Bezug auf lhren Namen verschlüsselt und dann ausgewertet; nur innerhalb der Klinik kann eine persönliche Zuordnung erfolgen. Nach Abschluss der Auswertung oder falls Sie nicht weiter an der Studie teilnehmen möchten, werden alle Daten gelöscht.

Wir erhoffen uns, durch diese Untersuchung einen wichtigen Beitrag zur gezielten Behandlung schwerkranker Patienten und betagter Menschen zu erhalten.

So kommt diese Untersuchung Ihnen und auch anderen Patienten zugute.

Bitte fragen Sie uns, wenn Sie die Information nicht verstehen oder mehr zu der beschriebenen Untersuchung wissen möchten: Prof. Dr. Friedemann Nauck, Abtelung Palliativmedizin, Universitätsmedizin Göttingen, Robert Koch Str. 40, 37075 Göttingen, Tel. 0551-3910501.

Mit herzlichem Dank für Ihre Mitwirkung.

Prof. Dr. Friedemann Nauck

Direktor der Abteilung Palliativmedizin 


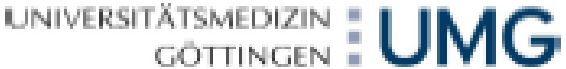

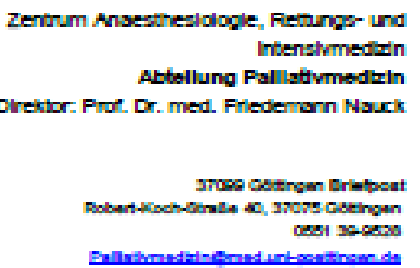

Patienteninformation Betreuer/in

Sehr geehrte Damen und Herren,

wir möchten Sie informieren über eine wissenschaftliche Studie unserer Klinik zum Thema

Symptome von Haut und Schleimhäuten

und ihre zugrundeliegenden mikrobiologischen Befunde

(Pravalenz und Reievanz enoraier Mykosen und Dermatomykosen in genlatrischen, intensivmedizin/schen, pallativmedizinlschen und pfregerischen Versorgungselnrichtungen-ein Survey).

In der Behandlung schwerstkranker und betagter Patienten stehen die Wiederherstellung und der Erhalt der Lebensqualität ganz im Vordergrund der therapeutischen Bemūhungen. Beschwerden im Mund und Rachenraum und auf der Haut sind als häufiges, belastendes Problem anzutreffen. Bislang ist jedoch wenig bekannt über das tatsächliche Ausmaß dieser Problematik; es gibt Hinweise auf eine eventuell zugrundeliegende Besiedlung oder Infektion mit Pilzerregern, deren Behandlung zu einer effektiveren Linderung der Beschwerden führen könnte.

Daher möchten wir Sie um lhre Zustimmung im Sinne der von thnen betreuten Person zur Teilnahme an einer freiwilligen wissenschaftlichen Studie bitten, die etwaige Beschwerden in Mund- und Rachenraum und auf der Haut und Ihre gesundheitlichen Situation genauer erfragt. Ein Mitarbeiter der Universität möchte Ihnen (in Ihrem Zimmer) die Mundschleimhaut und die Haut der Leistenregion und zwischen den Zehen mit einem Wattetupfer abstreichen. Zudem wird von der von Ihnen betreuten Person ein kleines Stūck Fußnagel und eine Stuhlprobe erbeten. Falls die von lhnen betreute Person derzeit Patient auf der Intensivstation ist und Fieber hat, soll eine Blutkultur entnommen werden. Diese Proben werden in der Universitätsmedizin Göttingen auf eventuell vorhandene Erreger untersucht und anschließend vernichtet. 


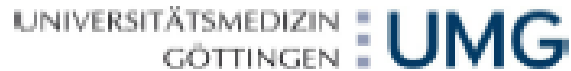

Die Untersuchung ist selbstverständlich freiwillig: bei Nichtteilnahme oder Widemuf entstehen Ihnen oder der von Ihnen betreuten Person keinerlei Nachteile. Die Untersuchung ist zudem schmerzfrei und ohne gesundheitliche Risiken. Die Untersuchungsergebnisse werden in Bezug auf den Namen der von lhnen betreuten Person verschlüsselt und dann ausgewertet; nur innerhalb der Klinik kann eine persönliche Zuordnung erfolgen. Nach Abschluss der Auswertung oder falls die von Ihnen betreute Person nicht weiter an der Studie teilnehmen möchte, werden alle Daten gelöscht.

Wir erhoffen uns, durch diese Untersuchung einen wichtigen Beitrag zur gezielten Behandlung schwerkranker Patienten und betagter Menschen zu erhalten.

So kommt diese Untersuchung uns allen zugute.

Bitte fragen Sie uns, wenn Sie die Information nicht verstehen oder mehr zu der beschriebenen Untersuchung wissen möchten: Prof. Dr. Friedemann Nauck, Abtelung Paliativmedizin, Universitätsmedizin Göttingen, Robert Koch Str. 40, 37075 Göttingen, Tel. 0551-3910501.

Mit herzlichem Dank für lhre Mitwirkung.

Prof. Dr. Friedemann Nauck

Direktor der Abteilung Palliativmedizin 




\section{Informationsblatt Kontrollgruppe}

Liebe Studentinnen,

Liebe Studenten,

wir möchten Sie informieren über eine wissenschaftliche Studie unserer Klinik zum Thema

Symptome von Haut und Schleimhäuten

und ihre zugrundeliegenden mikrobiologischen Befunde

(Pravalenz und Relevanz enoraler Mykosen und Dermatomykosen in gentatrischen, intensivmedizin/schen, pallativmedizinlschen und pfiegerischen Versorgungse/hnichtungen-ein Survey).

Wir möchten Sie um Ihre Zustimmung zur Teilnahme an einer freiwilligen wissenschaftlichen Studie bitten, die etwaige Beschwerden in Mund- und Rachenraum und auf der Haut genauer erfragt.

Beschwerden im Mund und Rachenraum und auf der Haut sind als häufiges, belastendes Problem bei schwerstkranken und betagten Patienten anzutreffen.

Es gibt Hinweise auf eine eventuell zugrundeliegende Besiedelung oder Infektion mit Pilzerregern, deren Behandlung zu einer effektiven Linderung der Beschwerden führen könnte.

Im Rahmen des Mikrobiologischen Unterrichtes werden Abstriche Ihrer Mundschleimhaut angefertigt. Durch diese Proben möchten wir untersuchen, ob Unterschiede der Mundflora verschiedener Altersgruppen festzustellen sind. Diese Proben werden anschließend vernichtet.

Die Untersuchung ist selbstverständlich freiwillig: bei Nichtteilnahme oder Widerruf 


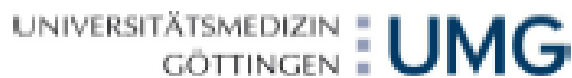

entstehen Ihnen keinerlei Nachteile. Die Untersuchung ist zudem schmerzfrei und ohne gesundheitliche Risiken. Die Untersuchungsergebnisse werden vollständig anonymisiert ausgewertet. Nach Abschluss der Auswertung werden alle Daten gelöscht.

Wir erhoffen uns, durch diese Untersuchung einen wichtigen Beitrag zur gezielten Behandlung schwerkranker Patienten und betagter Menschen zu erhalten.

So kommt diese Untersuchung uns allen zugute.

Bitte fragen Sie uns, wenn Sie die Information nicht verstehen oder mehr zu der beschriebenen Untersuchung wissen möchten: Prof. Dr. Friedemann Nauck, Abteilung Palliativmedizin. Universitätsmedizin Göttingen, Robert Koch Str. 40, 37075 Göttingen. Tel. 0551-3910501.

Mit herzlichem Dank für lhre Mitwirkung.

Prof. Dr. Friedemann Nauck

Abteilung Palliativmedizin

Prof. Dr. Uwe Groß

Abt. Medizinische Mikrobiologie

Dr. Bernd Alt-Epping

Abteilung Palliativmedizin

G. Ungermann, K.P. Wojak

Med. Fakultät 


\subsection{Einverständniserklärungen (Probanden, Betreuer und Kontrollgruppe)}

\section{UNIVERSITATSMEDIZIN $=\mathbf{U M G}$
GOTTINGEN $=\mathbf{M G}$}

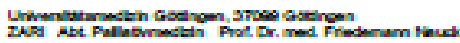

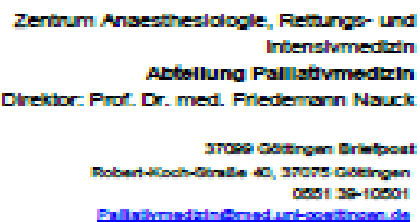

\section{Einverständniserklärung}

zur wiss. Studie: Symptome von Haut und Schleimhäuten

und ihre zugrundeliegenden mikrobiologischen Befunde

(Arbertsttel: Pravalenz und Relevanz enovaler Mywosen und Dermatomykosen in gertatrischen, pallathmedizhischen, intensivmedizhischen und pifegenischen Versorgungsberelchen - eh survey)

Sehr geehrte Patientin, sehr geehrter Patient,

Wir möchten lhnen sehr für lhre Mitarbeit danken!

Die von Ihnen erhobenen Daten unterliegen der ärztlichen Schweigepflicht gemäß \$203 StGB und sind den Bedingungen des Bundesdatenschutzes unterworfen.

Ihre Daten sind vor mutwilligem Zugriff geschützt und werden verschlüsselt, also ohne lhren Namen, Geburtsdatum usw. erfasst.

Einverständniserklärung:

- Ich bin mündlich und schriftich über die Ziele sowie den Ablauf der Untersuchung informiert worden.

- Ich habe die zur oben genannten Untersuchung schriftiche Patienteninformation erhalten und verstanden. Meine Fragen im Zusammenhang mit der Telnahme sind mir zufrieden stelend beantwortet worden.

- Ich nehme an dieser Studie freiwillig teil. Ich kann jederzeit und ohne Angabe von Gründen meine Zustimmung zur Teilnahme widerrufen; daraus entstehen mir keine Nachteile. Meine Daten werden dann gelöscht.

Name

Göttingen, den. 


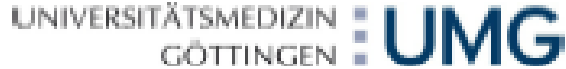

\section{Einverständniserklärung (Betreuer/in)}

zur wiss. Studie: Symptome von Haut und Schleimhäuten

und ihre zugrundeliegenden mikrobiologischen Befunde

(Avbertstitel: Prdvalenz und Relevanz enoraler Mywosen und Dermatomykosen in gertatrischen, pallabvmedizhischen, intensivmedizhischen und phegenischen Versorgungsberelchen - eln survey)

Sehr geehrte Betreuerin, sehr geehrter Betreuer.

Sie sind gebeten worden, im Sinne der von Ihnen betreuten Person Ihr Einverständinis zu der oben genannten wissenschaftichen Untersuchung zu geben. Wir möchten Ihnen sehr für lhre Mitarbeit danken!

Die von der von Ihnen betreuten Person erhobenen Daten unterliegen der ärztlichen Schweigepflicht gemäß §203 StGB und sind den Bedingungen des Bundesdatenschutzes unterworfen. Die Daten sind vor mutwilligem Zugriff geschützt und werden verschlüsselt, also ohne Namen, Geburtsdatum usw. erfasst.

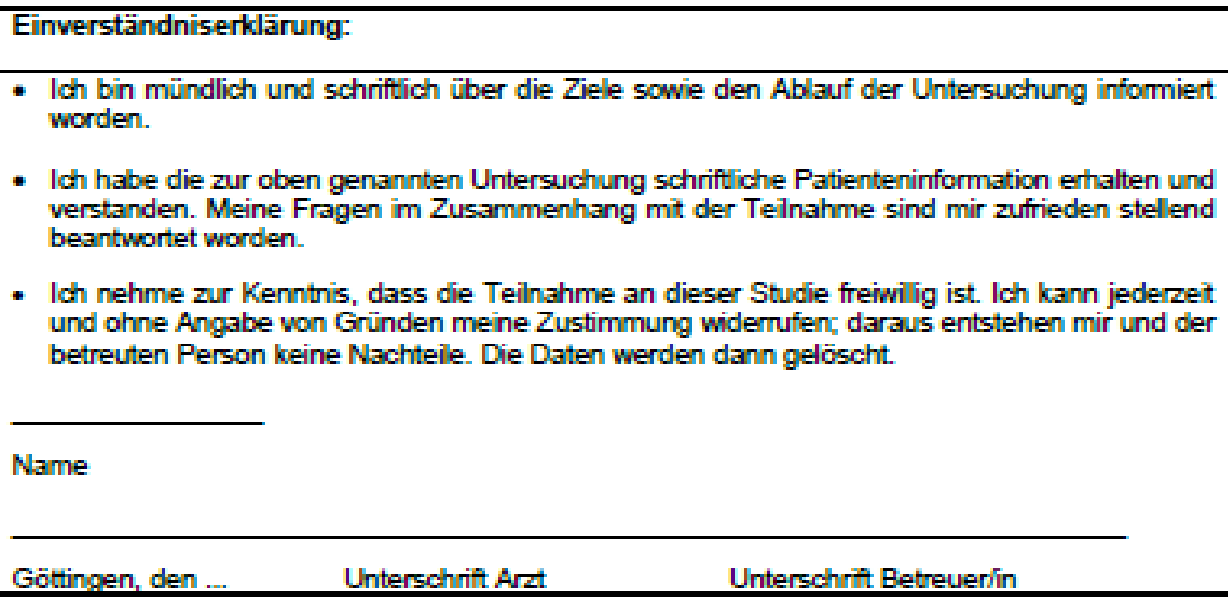




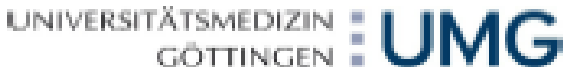

Zentum Anseathealologle, Retungs- und Intengtrmedistin ALtollung Pallistlvmedizin Direktor. Prot. Dr. med. Fredentonn Naxk

3roes geetrem probeat

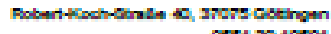

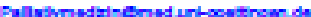

\section{Einverständniserklärung}

zur 'wiss. Studie: Symptome von Haut und Schleimhäuten

und ihre zugrundeliegenden mikrobiologischen Befunde

(Arbertsttel: Pravalenz und Relevanz enovaler Mykosen und Dermatomykosen in gertatrischen, palWattumedizhischen, intensivmedizhischen und pilegerischen Versorgungsberelchen - eln Survey)

Liebe Studentinnen,

Liebe Studenten,

Die von Ihnen erhobenen Daten unterliegen der ärztlichen Schweigepflicht gemäß \$203 StGB und sind den Bedingungen des Bundesdatenschutzes unterworfen.

Ihre Daten sind vor mutwiligem Zugriff geschützt und werden vollständig anonymisiert, also ohne lhren Namen, Geburtsdatum usw. erfasst.

\section{Einverständniserklārung:}

- Ich bin mündlich und schriftich über die Ziele sowie den Ablauf der Untersuchung informiert worden.

- Ich habe die zur oben genannten Untersuchung schriftiche Patienteninformation erhalten und verstanden. Meine Fragen im Zusammenhang mit der Telnahme sind mir zufrieden stellend beantwortet worden.

- Ich nehme zur Kenntris, dass die Teilnahme an dieser Studie freiwi ig ist. Ich kann jederzeit und ohne Angabe von Gründen meine Zustimmung widerrufen; daraus entstehen mir und der betreuten Person keine Nachteile. Die Daten werden dann gelöscht.

Name

Göttingen, den ... Unterschrift 


\subsection{Gliederung zur Datenanalyse}

\section{Übersicht:}

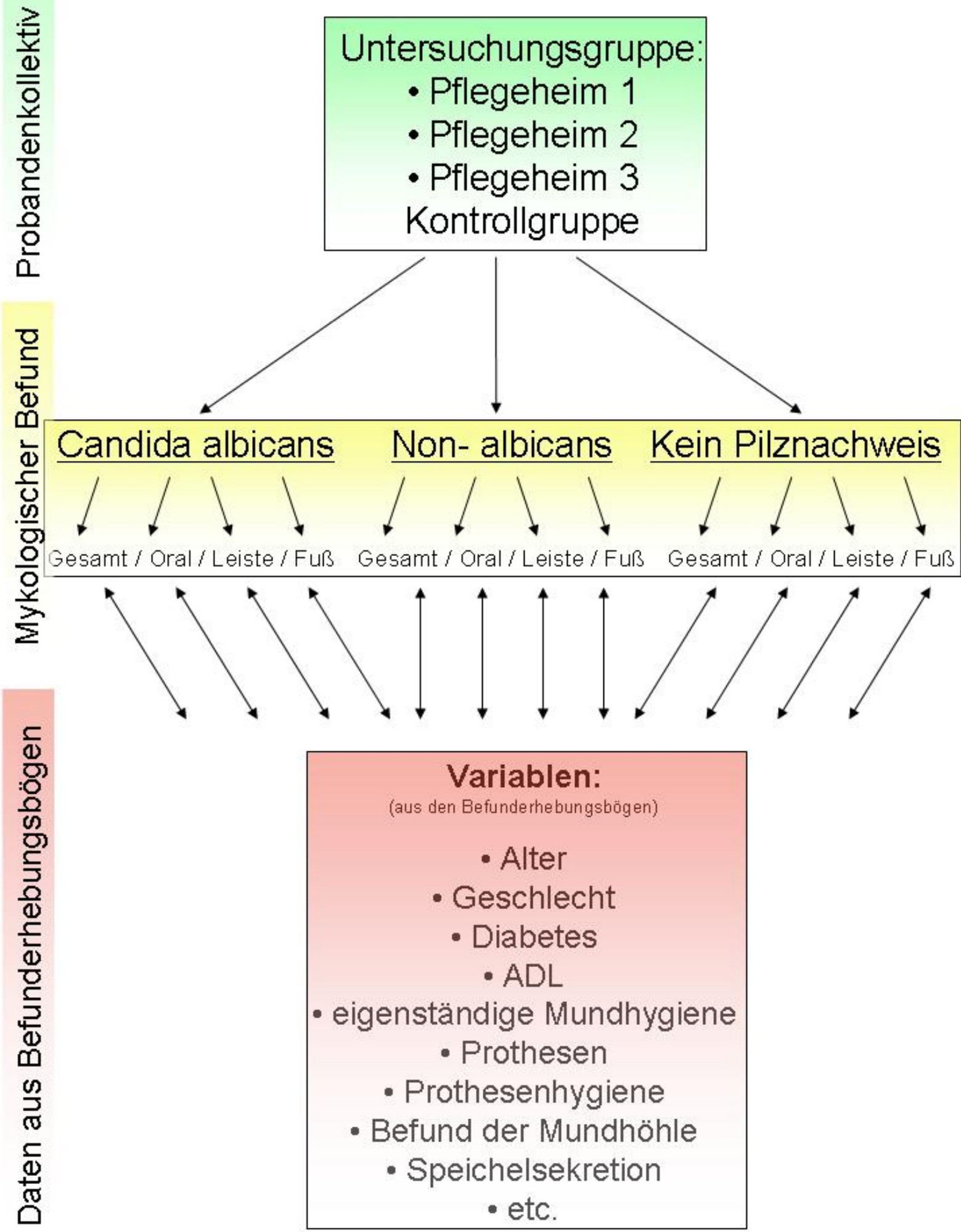




\section{Danksagung}

An erster Stelle möchte ich Herrn Prof. Dr. med. F. Nauck für konstruktive Unterstützung, die sehr hilfreichen Korrekturvorschläge und die Vergabe des Arbeitsthemas sowie Herrn Prof. Dr. med. U. Groß für die Hilfe sowie Förderung des Projektes danken, denn ohne sie wäre diese Arbeit nicht zustande gekommen.

Besonderer Dank gilt PD Dr. Bernd Alt-Epping, welcher mir die Möglichkeit gab, wissenschaftliches Arbeiten zu erlernen. Mich freute es zu jeder Zeit, dass ich als aus der Zahnmedizin stammender Student mit Herrn Alt-Epping an der Seite in anderen medizinischen Bereichen und Abteilungen arbeiten und forschen zu dürfen. Ich fühlte mich stets wohl und sehr gut betreut.

Ohne die gute abteilungsübergreifende Zusammenarbeit der Klinik für Palliativmedizin und der Abteilung Medizinische Mikrobiologie in dieser groß angelegten Studie wäre diese Arbeit nicht zustande gekommen. Großer Dank gilt in besonderem Maße Dr. Oliver Bader für die Betreuung, die geduldige Einarbeitung in der Mikrobiologie und die Hilfe bei der Auswertung der Proben.

Dem Nationalen Referenzzentrum für Systemische Mykosen sowie dessen vielen Mitarbeiterinnen und Mitarbeitern danke ich für die stetige Hilfe sowie für die Nutzung der Labore und Materialien für die Untersuchung und Auswertung der gesammelten Proben.

Herrn PD Dr. K. Jung aus dem Institut für Medizinische Statistik gilt mein Dank für die Beratung und Hilfe bei der Auswertung der statistischen Daten.

Darüber hinaus möchte ich mich bei den drei Göttinger Pflegeheimen, deren Geschäftsleitung und dem Pflegepersonal sowie den Probanden für die Mitarbeit bedanken. Ohne Ihr Mitwirken wäre diese Arbeit nicht zustande gekommen.

Ein besonderer Dank gilt meiner wissenschaftlichen Kollegin und guten Freundin G. Ungermann für die vielen, aber angenehmen Arbeitsstunden, die wir gemeinsamen in den Laboratorien der Mikrobiologie verrichtet haben. 


\section{Lebenslauf:}

Ich wurde als erster Sohn der Eheleute Prof.h.c. Universität Nanjing (VRC) Dr. med. dent. Winfried Wojak und Susanne Wojak, geb. Richter, am 03.März 1986 in Detmold geboren.

Eingeschult wurde ich 1992 in die Weerth-Grundschule Detmold und besuchte ab 1996 das Gymnasium Leopoldinum, ebenfalls in Detmold. Dort erlangte ich 2005 die allgemeine Hochschulreife.

Den Studiengang Zahnmedizin begann ich im April 2006 an der Georg-August Universität Göttingen. Die Naturwissenschaftliche Vorprüfung legte ich im Februar 2007 sowie die Zahnärztliche Vorprüfung im September 2008 mit der Gesamtnote "gut" ab. Im Juni 2011 schloss ich die universitäre Ausbildung in Regelzeit mit dem Bestehen des Zahnärztlichen Staatsexamens mit der Abschlussnote „sehr gut" ab und erhielt meine Approbation als Zahnarzt.

Die Arbeit zur Dissertation begann Ende 2009, zusammen mit G. Ungermann.

Im Zeitraum von Februar 2012 bis Februar 2014 arbeitete ich in der zahnärztlichen Gemeinschaftspraxis „Zahnmedizinisches Zentrum am ZOB Reinecken, Waller \& Kollegen“ in Buxtehude sowie in der Zahnarztpraxis "Reinecken \& Kollegen“ in Moisburg als Ausbildungs- bzw. Vorbereitungsassistent.

Von März 2014 bis Juni 2015 arbeitete ich in beiden o.g. Praxen als angestellter Zahnarzt und absolvierte neben der zahnärztlichen Arbeit und der Arbeit zur Dissertation das Curriculum „Funktionsdiagnostik und Therapie“ in Hamburg.

Seit September 2015 arbeite ich in der Praxis am Sande in Lüneburg als angestellter Zahnarzt. 